\title{
Parents living within the paradoxes of infant death from life- limiting anomaly: A grounded theory study
}

Susan Bush Welch

West Virginia University

Follow this and additional works at: https://researchrepository.wvu.edu/etd

\section{Recommended Citation}

Welch, Susan Bush, "Parents living within the paradoxes of infant death from life-limiting anomaly: A grounded theory study" (2012). Graduate Theses, Dissertations, and Problem Reports. 488.

https://researchrepository.wvu.edu/etd/488

This Dissertation is protected by copyright and/or related rights. It has been brought to you by the The Research Repository @ WVU with permission from the rights-holder(s). You are free to use this Dissertation in any way that is permitted by the copyright and related rights legislation that applies to your use. For other uses you must obtain permission from the rights-holder(s) directly, unless additional rights are indicated by a Creative Commons license in the record and/ or on the work itself. This Dissertation has been accepted for inclusion in WVU Graduate Theses, Dissertations, and Problem Reports collection by an authorized administrator of The Research Repository @ WVU.

For more information, please contact researchrepository@mail.wvu.edu. 


\title{
PARENTS LIVING WITHIN THE PARADOXES OF INFANT DEATH FROM LIFE-LIMITING ANOMALY: A GROUNDED THEORY STUDY
}

\author{
Susan Bush Welch \\ Dissertation submitted to the \\ School of Nursing \\ at West Virginia University \\ in partial fulfillment of the \\ requirements for the degree of \\ Doctor of Philosophy \\ in \\ Nursing
}
Alvita K. Nathaniel, Ph.D., Chair Mary Jane Smith, Ph.D. Margaret A. Burkhardt, Ph.D. K. Joy Buck, Ph.D. Paula F. Taylor, M.D.
School of Nursing

Morgantown, West Virginia

2012

Key words: parents, neonates, infants, infants with disabilities, palliative care Copyright 2012 Susan Bush Welch 


\section{ABSTRACT \\ Parents Living Within the Paradoxes of Infant Death from Life-Limiting Anomaly: A Grounded Theory Study \\ Susan Bush Welch}

BACKGROUND: Life-limiting congenital anomalies are the leading cause of death of infants in the United States. Most of these infants die in intensive care units with limited access to adequate palliative/end-of-life care. There is an extensive knowledge gap about the experience of the parent of infants who died from life-limiting anomaly. PURPOSE: The aims of the study was to explore the process a parent goes through as they experience the birth, life and ultimate death of an infant from a life-limiting explore the process a parent goes through as they experience the birth, life and ultimate death of an infant from a life-limiting congenital anomaly and (b) develop a substantive theory to describe the process parenting an infant with a lifelimiting congenital anomaly from birth to death. METHODS: Classic grounded methodology was used to analyze field notes of open-ended interviews with 11 parents whose infant died from a life-limiting congenital anomaly between the ages of 48 hours and 15 months. FINDINGS: The grounded theory Parents Living within the Paradoxes of Infant Death from Life-Limiting Anomaly describes the experience of parents from pregnancy to life after the infant's death. The theory contains three stages and two cutting points. The first stage of the theory is living in innocence in which the pregnant/expectant parent anticipates or has a healthy baby. This stage ends with the first cutting point of getting the bad news or getting the diagnosis of a life-limiting anomaly. The second stage is being a good mom/dad in the new reality in which the parent experiences being the parent of a baby expected to die from a life-limiting anomaly. The second stage ends with the second cutting point, death of the baby. The final stage of the theory is going on describes how a parent goes on with life after the death of the baby. CONCLUSIONS: Parents living within the paradoxes of infant death from life-limiting anomaly has great implications for nursing in education, practice and research. This new grounded theory has the potential to assist in improving palliative and end-of-life care for infants. 


\section{DEDICATION}

I dedicate this to all the beautiful little souls who spent so little time on earth, but who live on forever in the hearts and minds of those left behind. 


\section{ACKNOWLEDGEMENTS}

There are many who helped me through this long dissertation process. It is with a grateful heart that I acknowledge those who accompanied me on this journey.

I want to thank the chair and members of my committee for their guidance through the dissertation process. Dr. Alvita Nathaniel, chair of the committee, was always generous with her time, support, knowledge and feedback through the numerous drafts, personal meetings and phone calls. Classic grounded theory became alive to me through her mentoring. She is a nursing scholar in the truest sense. Dr. Mary Jane Smith, my first professor in the Ph.D. program, was open and allowed me to pursue a concept of which she was unfamiliar, a concept which ultimately became the theoretical framework for this study. Dr. Peggy Burkhardt was always positive and provided invaluable feedback. Dr. Joy Buck shared her extensive background in palliative care. Dr. Paula F. Taylor, a palliative care physician, provided clinical expertise and the means to recruit enough participants to complete this dissertation.

This dissertation would not exist without the brave participants who shared their compelling and tragic stories. I am in awe of their ability to function day-to-day after such a great loss, the death of their baby. These parents' strength is evidence of the indomitable spirit of human beings. It is my hope that this work honors the trust they exhibited by telling me their story.

Without the assistance of Hospice Care and the Trisomy 18 Foundation there would be no results to report. The assistance of these two organizations in recruitment of participants was invaluable. Dr. June Lunney, my first Ph.D. advisor, shared her extensive knowledge of end-oflife care and helped me refine the topic of this dissertation. My colleagues at the Marshall 
University School of Nursing were encouraging and supportive over the many years it took to complete this work.

Special recognition goes out to a very special group of women, my cohort in the Ph.D. program: Dr. Sara Clutter, Dr. Jill Cochran, Dr. Sue Coyle, Dr. Denise Lucas, and Dr. Jennifer Presley-Ice. We shared frustrations and celebrated successes during this journey called doctoral studies. Your friendship sustained me. The laughter, well the laughter made the journey tolerable and at times fun. I am proud to call you my colleagues and friends.

Finally, I wish to express my gratitude to my wonderful family. I am grateful for parents who told me I could do anything and modeled a strong work ethic. Robyn, my beautiful and talented daughter, exhibited great tolerance over the years about mom's "second career" of professional student. Thank you, Robyn, for your understanding and encouragement. Lastly, I want to share my gratitude to Jack, my husband and best friend. He too has tolerated and supported my many forays into higher education. Through it all he has always been my best supporter and nag (in a loving way of course). Honey, I think I am finally done with going back to school. 


\section{TABLE OF CONTENTS}

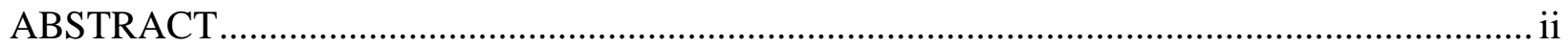

DEDICATION

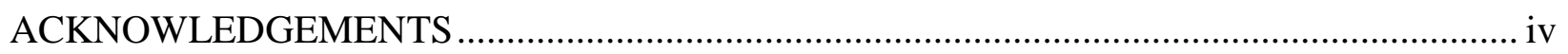

CHAPTER ONE: STATEMENT OF THE PROBLEM ........................................................ 1

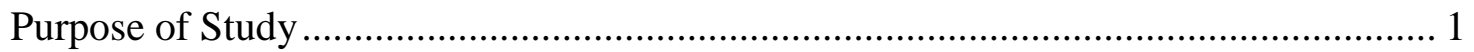

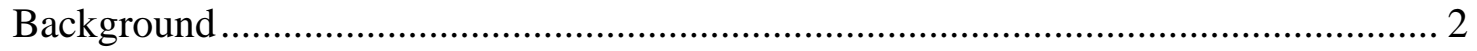

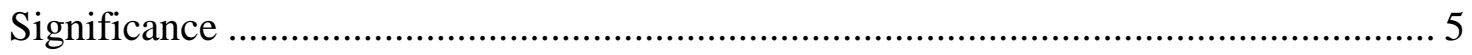

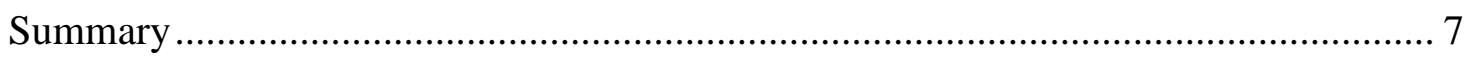

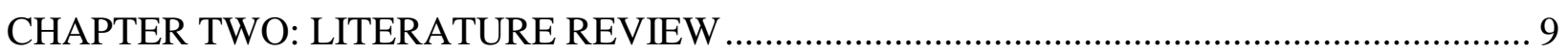

Conceptual Foundation: Good Death ..................................................................... 10

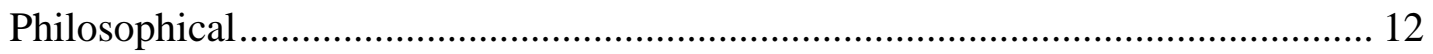

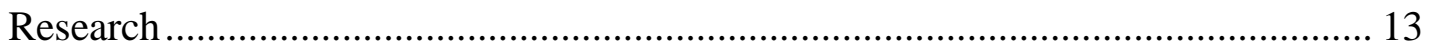

Seminal Reports and Position Statements on Pediatric Palliative and End-of-Life Care .................................................................................................. 17

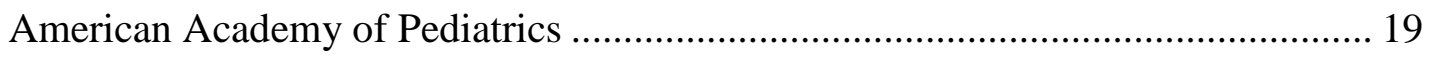

National Hospice and Palliative Care Organization ............................................... 20

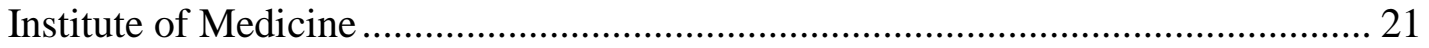

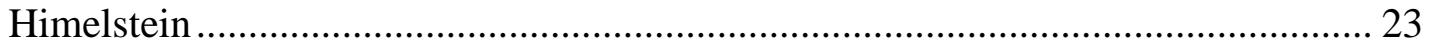

Neonatal and Infant Palliative and End-of-Life Care ............................................. 25

Neonatal Palliative Care Protocols and Programs ................................................ 27

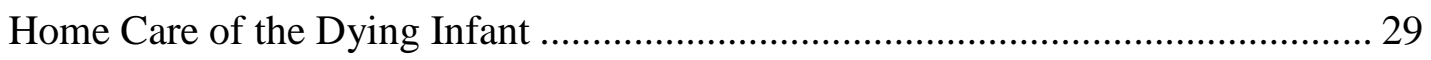

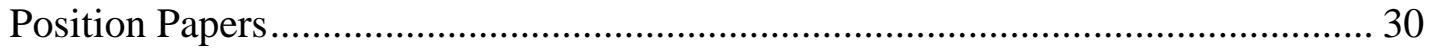

Palliative Care for Neonates/Infants with Life-Threatening

Congenital Anomalies....................................................................................... 31

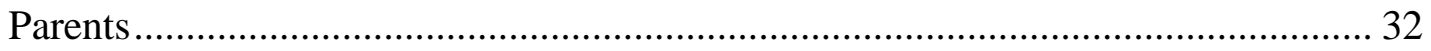

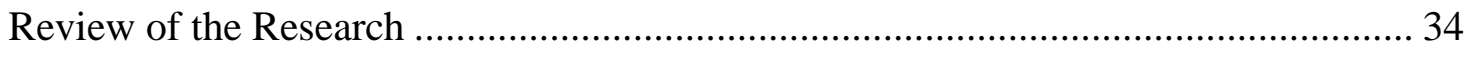

Neonatal and Infant Palliative and End-of-Life Care ............................................. 34

Parents and Neonatal and Infant Palliative Care ...................................................... 38

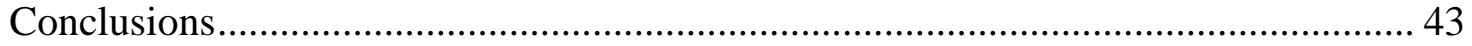

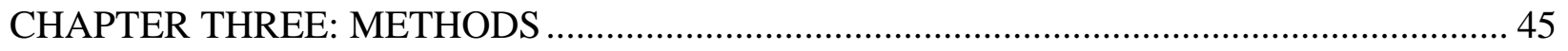

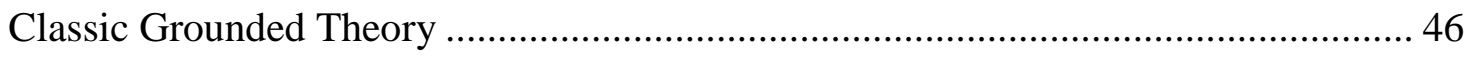

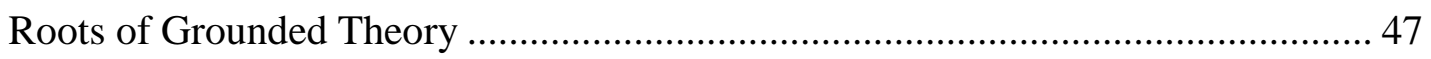




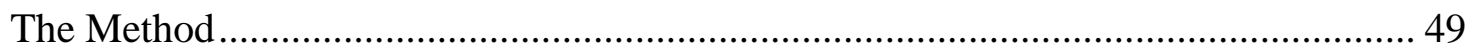

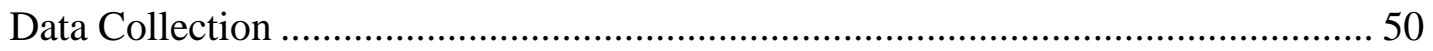

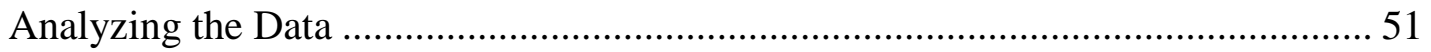

Theoretical Sampling .......................................................................................... 51

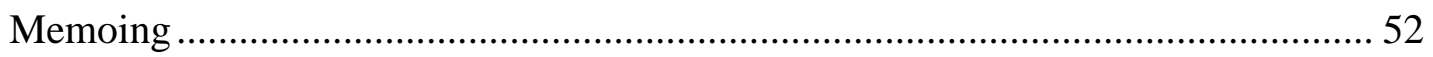

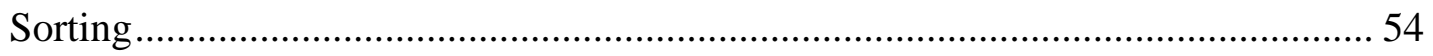

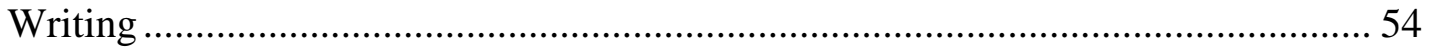

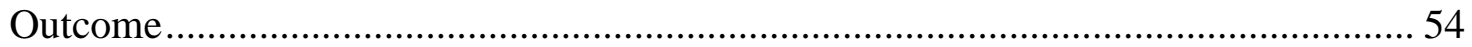

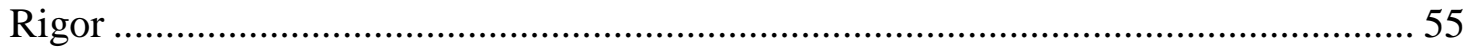

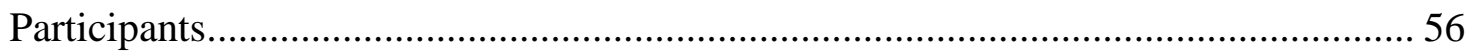

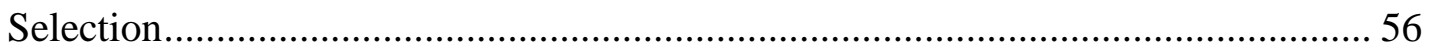

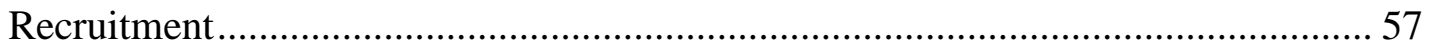

Characteristic of Participants ................................................................................... 59

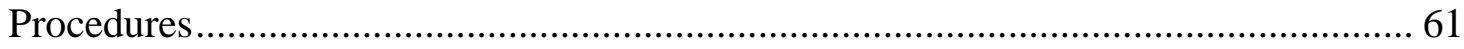

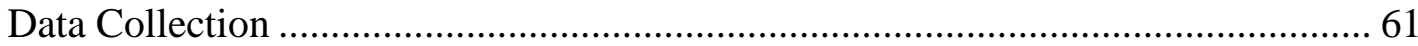

Analysis..................................................................................................... 62

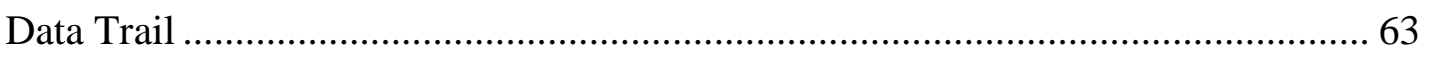

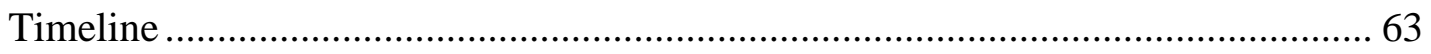

Protection of Participants ..................................................................................... 63

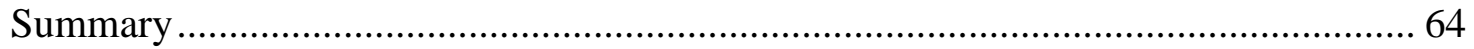

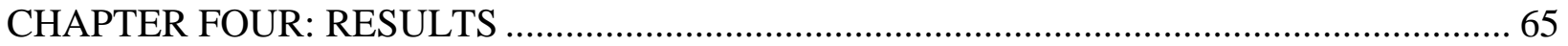

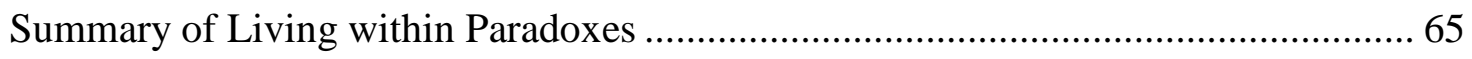

Parents Living within the Paradoxes of Infant Death from

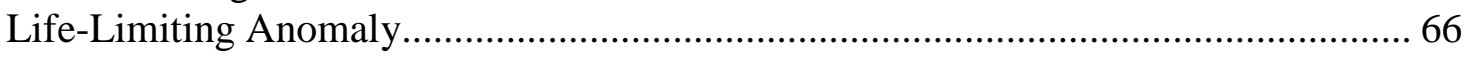

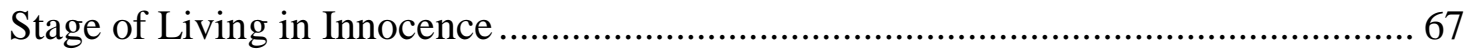

Cutting Point One: Getting the Bad News.................................................................... 68

Stage of Being a Good Mom/Dad in the New Reality .................................................. 69

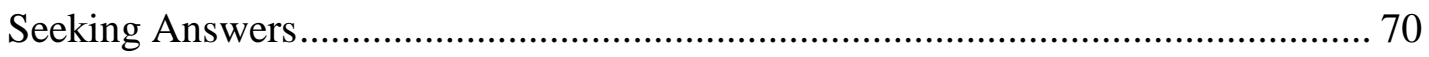

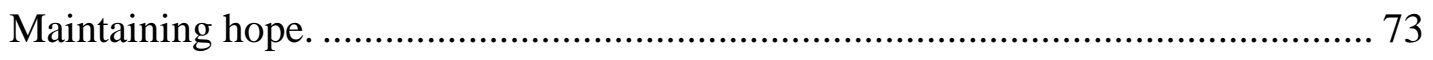

Squeezing a Lifetime into a Moment....................................................................... 77

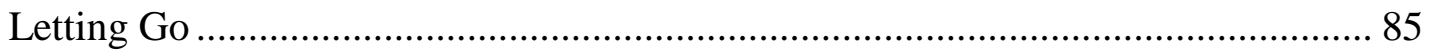

Cutting Point Two: The Death of the Baby .............................................................. 86

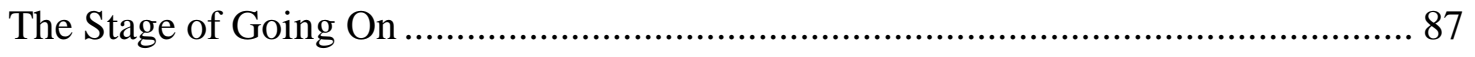


Seeking Meaning in the Baby’s Life ......................................................................... 90

Keeping the Baby’s Memory Alive ........................................................................... 92

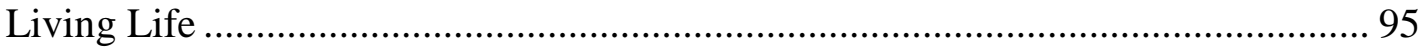

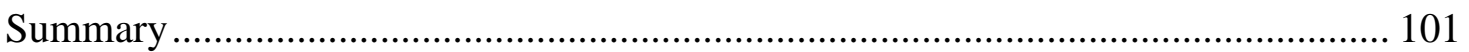

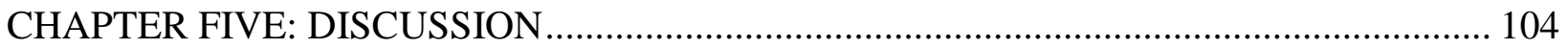

Summary of Living within Paradoxes ................................................................... 104

Comparison of the Theory with the Literature ............................................................. 105

Empirical Research Literature ................................................................................. 105

Theoretical Literature.......................................................................................... 112

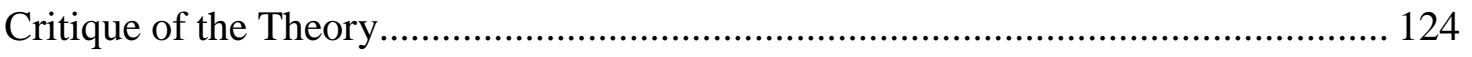

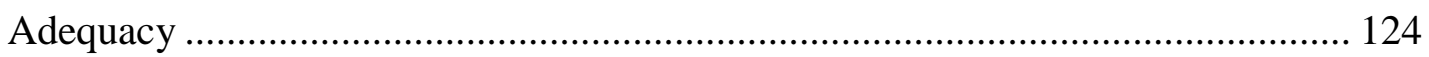

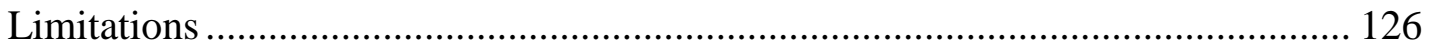

Implications for Nursing ....................................................................................... 127

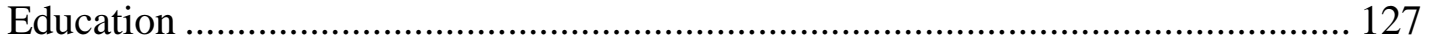

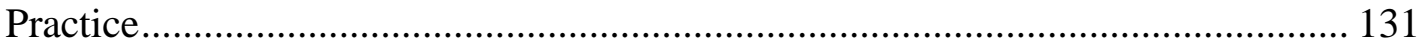

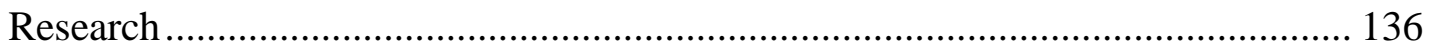

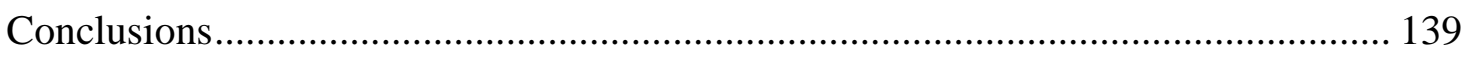

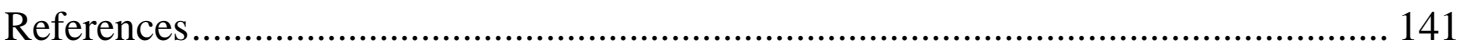

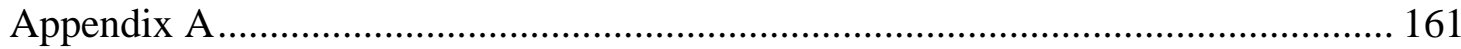

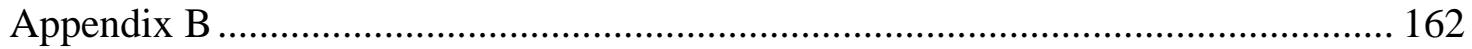




\section{LIST OF FIGURES}

Figure 1. Parents Living within the Paradoxes of Infant Death from Life-Limiting Anomaly ............................................................................66

Figure 2. Stage of Being a Good Mom/Dad in the New Reality ............................................70

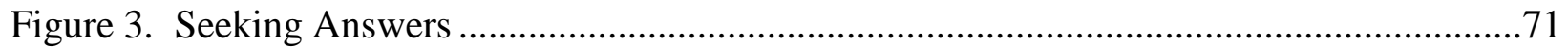

Figure 4. Squeezing a Lifetime into a Moment ..................................................................78

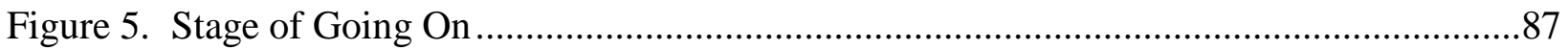

Figure 6. Seeking Meaning in the Baby’s Life ..................................................................91

Figure 7. Keeping the Baby’s Memory Alive......................................................................93

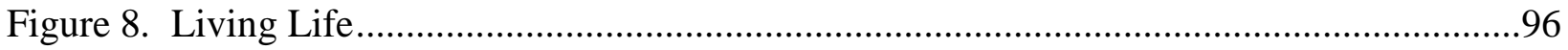




\section{CHAPTER ONE: STATEMENT OF THE PROBLEM}

Birth and death are two end-points on the continuum of life. Most envision these points as widely separated with many years of life filling the space between the two ends. For some children that space separating birth and death is very close with death occurring just minutes after birth. Many of these children are infants born with life-limiting congenital anomalies. The recent literature in pediatrics has focused on good death and adequate palliative and end-of-life care for infants with life-limiting congenital anomalies and the families. Unfortunately, little empirical evidence defines and describes adequate palliative and end-of-life care. Of special note is the scarcity of evidence describing parents' experiences during these times. Since parents are integral to infants' end of life and palliative care, this study explored the experience of parents of infants and children who have died because of life-limiting congenital anomalies

\section{Purpose of Study}

Parents play a vital role during the limited life span and ultimate death of their infant with life-limiting congenital anomalies. Thus, it is important for health care professionals to understand the experience of the parents in order to be partners in provision of optimal palliative care. The purpose of this study was to: (a) explore the process a parent goes through as they experience the birth, life and ultimate death of an infant from a life-limiting congenital anomaly and (b) develop a substantive theory to describe the process of parenting an infant with a lifelimiting congenital anomaly from birth to death. The development of a substantive theory using classic grounded theory methodology will assist health care professionals in understanding parents' needs through the process of birthing, caring for, and losing an infant with a life-limiting congenital anomaly. 


\section{Background}

In 2009, almost 45,000 children died in the United States (Kochanek, Xu, Murphy, Miniño, \& Kung, 2011). Children under the age of one year, neonates (less than 28 days old) and infants (29 days old to 1 year of age), comprise half of the total number of deaths in childhood (Field \& Behrman, 2003; National Hospice and Palliative Care Organization [HPCO], 2001). The data from 2009 suggest the number is closer to 58\%, with 26,412 deaths occurring in the first year of life (Kochanek et al., 2011). Complications of prematurity, trauma (accidental and non-accidental), sudden infant death syndrome (SIDS), infection and congenital anomalies are just a few of the causes of neonatal and infant mortality.

Some authors estimate that congenital anomalies account for $20-50 \%$ of the mortality of neonates and infants (Feudtner et al., 2002; Feudtner, Silveira, \& Christakis, 2002; Field \& Behrman, 2003; Mathews, MacDorman, \& Menacker, 2002; Robison, Hodgman, Barton, \& Pavlova, 2003). Data from the "National Vital Statistics Report” indicate that $20 \%$ of infant deaths $(n=5,319)$ resulted from congenital anomalies in 2009 (Kochanek et al., 2011). Therefore, congenital anomalies are the primary cause of neonatal and infant mortality in the United States. Chromosomal anomalies such as Trisomy 13 or 18 tend to be the most lethal for neonates and infants; 50\% of infants with chromosomal anomalies die before their first birthday (Dastgiri, Gilmour, \& Stone, 2003). Disorders to major systems of the body such as congenital heart defects, diaphragmatic hernia, and renal anomalies have variable lethality when they occur in isolation; however the co-presence of a chromosomal anomaly increases the risk of mortality (Brackley, Kilby, Wright, \& Brawn, 2000; Chandra \& Spitzer, 2001; Eronen, 1997; Scott, 2002). Great variability in life span exists in neonates and infants with congenital anomalies; some die quickly within hours of birth, while others live almost a full year (Dastgiri et al., 2003). 
The setting where these neonates and infants live and eventually die is of prime concern. Approximately 90\% of neonates and infants with congenital anomalies die in hospitals and most die in neonatal (NICU) or pediatric (PICU) intensive care units (Carter et al., 2004; Brandon, Docherty, \& Thorpe, 2007; Feudtner et al., 2002; Leuthner, Boldt, \& Kirby, 2004; Zwerdling et al., 2000). Feudtner et al. (2011) found that $62.1 \%$ children who received palliative care consultation in six large pediatric hospitals died in the hospital and 56\% of those died in an ICU. Not only is the setting where these neonates and infants die important, but so is the type of care given. According to a 2002 press release by Last Acts, a national coalition to improve care and caring near the end of life, only $9 \%$ of dying children receive hospice services. These statistics are congruent with findings by Leuthner, et al (2004) in a study of death of infants in Wisconsin and of demographic data of children enrolled in Star Shine, the hospice of Children's Hospital Medical Center in Cincinnati, Ohio (Zwerdling et al, 2000). Documentation of provision of palliative care or end-of-life care interventions only occurred in $23 \%$ and $6 \%$ respectively of reviewed charts (Brandon, Docherty, \& Thorpe, 2007). Friebert and Huff (2009) estimated that only $10-20 \%$ of dying children received hospice services and that a similar percentage of children with life-threatening conditions received palliative care. Infants only comprised 22.2\% of the pediatric clientele of hospices (Friebert, 2009). Feudtner et al. (2011) found that only $17.1 \%$ of the patients who received a pediatric palliative care consult were infants, despite the fact that most pediatric deaths occur in the first year of life. Therefore, only a small minority of the almost 5,300 infants who die each year of congenital anomalies receive palliative care or hospice services.

Babies with life-limiting congenital anomalies receive less than optimal palliative and end-of-life care. This lack of adequate palliative care is a significant clinical problem that 
requires action by nursing. Inadequate access to palliative care has a great impact on individuals, families and society as a whole. Negative consequences of inadequate access to palliative care include increased pain and suffering as well as increased economic costs.

Dying infants who receive palliative care consultation suffer less pain and suffering because of a decrease in futile, invasive procedures than those without consultation (Pierucci, Kirby, \& Leuthner, 2001). Conversely, inadequate palliative care for dying infants contributes to pain and suffering experienced during a final hospitalization as those infants experience invasive procedures (Carter et al, 2004; Feudtner, DiGiuseppe, \& Neff, 2003; Feudtner et al., 2002). Parents, siblings, other family members and health care professionals suffer emotionally and often physically after the death of a child (Adams, Corr. Davies \& Deveau, 1999; Black, 1998; Costello \& Trinder-Brook, 2000; Downey, Begiamin, Heuer \& Juhl, 1995; Kazak \& Noll, 2004; Li, Hansen, Precht, Mortensen \& Olsen, 2003; Milo, 1997; NHPCO, 2001; Papdatou et al, 2002; Salomon, Belouet, Vinant-Binam, Discard \& Vidal-Trocan, 2001; Wood \& Milo, 2001; Yam, Rossiter \& Cheung, 2001). Researchers at the NHPCO found effective support and bereavement services, such as offered by hospices, decreased psychological distress of surviving families and decreased medical costs due to somatization.

The care of dying children incurs direct and indirect economic costs. Direct costs include the hospital charges for the room, equipment, medications and procedures as well as physician charges. Indirect costs include items related to receiving care away from home such as lodging, gas, food and lost wages (NHPCO, 2001). The type and site of care affects both direct and indirect costs. Palliative and end-of-life care provided in the home rather than in the hospital is less expensive (Belasco, Danz, Drill, Schmid \& Burkey, 2000; NHPCO, 2001; Yoon et al, 1997). Conversely, aggressive medical care in an intensive care is the most expensive. Since most 
infants die in intensive care units, shifting site of care to regular pediatric units or to home could decrease both direct and indirect costs. These direct and indirect economic costs contribute to parents' burden at the end of a child’s life.

Many challenges impede quality palliative care for infants and children. The 2001 NHPCO report summarized the challenges that included: death not associated with childhood, medical and resource focus on cure versus palliation, conflicting obligations to child and family, palliation and curative treatments commonly seen as mutually exclusive, communication issues related to child's developmental status and the uncertainty in prognosis and time left for children (NHPCO, 2001). In addition, little empirical knowledge exists about pediatric palliative and hospice care, even less about palliative and hospice care for infants. Many of the assumptions about pediatric end-of-life care originated from adult data, which may be irrelevant. Andresen \& Toce (2002) identified lack of process and outcomes measurement tools as a significant barrier to improved pediatric palliative care.

Published statements, principles, protocols and research findings about pediatric palliative care have been surprisingly congruent. All addressed and or examined the need for adequate palliative and hospice care for children with life-threatening illnesses and their families. Extensive knowledge gaps exist regarding good death for children, even more so for neonates and infants with life-limiting anomalies. One finds that the most common phrases in the 2003 IOM report are "Research is limited” or "systematic data are not available” (Field \& Behrman, 2003). That phrase remains true today.

\section{Significance}

Parents play a pivotal role during the life and ultimate death of their infant with lifelimiting congenital anomalies. During the pregnancy, they dream of and celebrate the growing 
life. It is the parents who, upon diagnosis of anomaly, have to shift gears from dreaming of a bright future to a more limited one that includes the death of their baby. Parents provide care and caring to their dying infant.

The impact of the loss of a child on parents is much discussed and well-studied (Arnold \& Gemma, 2008; Barr \& Cacciatore, 2008; Barrera et al., 2007; Barrera et al., 2009; DeCinque et al., 2009; Keesee, Currier, \& Neimeyer, 2008; Meert, Briller, Schim, Thurston, \& Kabel, 2009; Rogers, Floyd, Seltzer, Greenberg, \& Jinkuk, 2008; Raphael, 2006; Toller, 2008). A body of literature and research exists that examines the parents' experience of loss of a baby. However, most of that research focuses on loss through sudden and unexpected means such as Sudden Infant Death Syndrome (SIDS), stillbirth, extreme prematurity or miscarriage (Carter, 2007; Gold, 2007, Kavanaugh \& Moro, 2006; Romesberg, 2004; Walden, Sudia-Robinson \& Carrier, 2001; Workman, 2001). Many of these studies included all parents whose baby died regardless of cause of death.

The experience of parents whose infant has a life-limiting congenital anomaly is different. These parents know their baby will die. However, when death will happen is unknown because of the great variability in life span among these infants (Dastgiri et al., 2001). Very few researchers specifically explored the experience of parents who had an infant die with life-limiting congenital anomalies after a live birth.

A substantial knowledge gap exists about the experience of parents who know their baby will die from life-limiting congenital anomalies. It is important for health care professionals to understand this experience in order to be partners in provision of optimal palliative care for these infants and their families. Thus, the need for more research to guide the development of interventions based upon sound evidence. 
This study explored the process of parenting as parents experience the birth, life and death of an infant with a life-limiting congenital anomaly through use of classic grounded theory methodology. A substantive theory to describe the parental process from birth to death was the outcome of the study. The development of a substantive theory using classic grounded theory methodology will assist health care professionals in understanding parental needs through the process of birthing, caring for, and losing an infant with a life-limiting congenital anomaly.

\section{Summary}

Every year over 26,000 neonates and infants die in the United States; congenital anomalies are the leading cause of those deaths (Field \& Behrman, 2003; Kochanek et al., 2011, NHPCO, 2001). A very small percentage of dying babies receive the benefit of palliative or hospice services and most die in intensive care units. Inadequate palliative care negatively affects the child, families, and society because of increased pain and suffering as well as increased direct and indirect economic costs. Demands for improved palliative care for children fill the pediatric literature and advocacy group web sites; some specifically examine the needs of neonates and infants

Extensive knowledge gaps exist regarding good death for children, even more so for neonates and infants with life-limiting congenital anomalies. "Research is limited” or “systematic data are not available” are the most common phrases in the 2003 IOM report When Children Die. More research is in order to identify appropriate end-of-life care for this most vulnerable population, the infant.

Parents of infants with life-limiting congenital anomalies, as primary providers of love and care, are a vital part of any discussion regarding optimal palliative and end-of-life care. Yet, little research specifically explores the experience of these parents. Sound evidence will allow 
health care providers to tailor effective interventions. Therefore, the purpose of this study was to (a) explore the process a parent goes through as they experience the birth, life and ultimate death of an infant from a life-limiting congenital anomaly and (b) develop a substantive theory to describe the process parenting an infant with a life-limiting congenital anomaly from birth to death. 


\section{CHAPTER TWO: LITERATURE REVIEW}

Historically, the death of a baby in the first year of life has been a common occurrence. The infant mortality rate at the beginning of the $20^{\text {th }}$ century in the United States was 100 infant deaths out of 1,000 live births (Center for Disease Control [CDC], 1999). One out of ten live born infants died. Improvements in environmental hygiene, pasteurization of milk, the introduction of immunizations and antibiotics and improvements in prenatal, perinatal and pediatric care over the century contributed greatly to the decline in the infant mortality rate in the United States over the last century (CDC, 1999). The infant mortality rate in 2006 in the United States was six deaths per 1,000 births in 2006 (National Center for Health Statistics, 2009). Despite the dramatic drop in infant mortality, neonates and infants continue to account for over half of the deaths in childhood in the United States (Field \& Behrman, 2003; Kochanek et al., 2011).

Today, congenital anomalies are the primary cause of infant mortality (Field \& Behrman, 2003; Kochanek et al., 2011). Infants with life-limiting congenital anomalies have a variable life span and may live almost a full year (Dastgiri et al., 2003). A vast majority, approximately $90 \%$, die in hospital, most in neonatal (NICU) or pediatric (PICU) intensive care units (Feudtner et al., 2002; Leuthner, Boldt, \& Kirby, 2004; Zwerdling et al., 2000). Conversely, only 9-20\% of dying children receive palliative and hospice services (Friebert \& Huff, 2009; Last Acts, 2002, Leuthner et al., 2004; Zwerdling et al., 2000).

The focus on palliative and end-of-life care for neonates and infants with life-limiting congenital anomalies is a recent phenomenon. Since scant research related to the phenomenon is in the published literature, a majority of the literature about pediatric and specifically neonatal and infant palliative and end-of-life care is still in the form of position papers and editorials. 
There is published research about dying children and the subsequent impact on parents, other family members and health professionals but the research rarely focuses solely on neonates and infants. Most of the published research specific to the death of an infant uses descriptive methodology, seeking to quantify the problem as well as understand the experience of the dying process for the infant and families.

Since there is limited research, organization of the review of the literature in this chapter follows the trend of the literature: (a) conceptual foundation, (b) seminal reports and position statements, (c) review of the research, and (d) conclusions. The first section of the literature review discusses good death, which is the conceptual foundation of this dissertation. Presentation of the seminal reports and position statements regarding pediatric palliative and hospice care and other writings on palliative care for neonates and infants and their parents provides background about pediatric, infant and neonatal palliative and end-of-life care and comprises the second section. The review of the research presents the published research about neonatal and infant palliative and hospice care, including research that explores the experience of parents of dying infants. The last section, conclusions, provides a summary of the state of the science about neonatal and infant palliative and hospice care, the gaps in that knowledge and the research questions that guide this study.

\section{Conceptual Foundation: Good Death}

Good death is the pivotal concept that underlies all the written and clinical work to improve end-of-life care. Health professionals who work with dying patients use the term frequently, since a good death is the ultimate goal of end-of-life care. It is not commonly used in connection to dying infants. The IOM report edited by Field and Cassel (1997) defined a good death, one quoted by many authors, as: 
A good death is one that is free from avoidable distress and suffering for patients, families and caregivers; in general accord with patients’ and families’ wishes; and reasonably consistent with clinical, cultural, and ethical standards (p. 4).

Good death provides the conceptual foundation of this study.

Historically, Plato’s descriptions of Socrates death in the Phado may form the modern foundation of good death (Walters, 2004). On his deathbed, Socrates tells the followers who surround him "there is great reason to hope that death is a good" (Plato, 2009, p. 60). The scene depicts a death where there is control, acceptance, comfort and the presence of loved ones. Walters (2004) opines that Socrates death, as written by Plato, is the "prototypical good death story” (p. 407).

Good death was the inherent objective in the Christian hospices founded in the $4^{\text {th }}$ century. These early hospices were a stark contrast to the Roman hospitals of the time. Those Roman hospitals functioned more as emergency rooms and were early models of the maxim “treat'em and street’em” (DeSpelder \& Strickland, 2002). The dying received special care and honor in the early hospices.

The modern dialogue on good death began in England as Dr. Cicely Saunders worked to create the first modern hospice, St. Christopher's Hospice, which opened in 1967. The dialogue came to the United States when Dr. Saunders visited the School of Nursing at Yale University in 1963 and again in 1966 (NHPCO, 2001). During the second meeting, Dr. Saunders met with Florence Wald, Elisabeth Kubler-Ross and other early leaders of the American movement to improve end-of-life care (DeSpelder \& Strickland, 2002). Development and establishment of the first hospice in the United States began with those meetings. 
Death as an appropriate topic for research began with the publication of the groundbreaking Awareness of Dying by Barney Glaser and Anselm Strauss in 1965. Glaser and Strauss observed how families interacted during a life-threatening illness of a family member. Those observations led to the grounded theory on the influence of awareness on communication with the dying. Four types of awareness (closed, suspected, mutual pretense and open) influenced how family members interacted with their dying loved one and in turn how the nursing staff interacted with the family and patient (Glaser \& Strauss, 1965).

Today, good death is the legitimate topic of scholarly discussion and inquiry. In the current literature, one finds two scholarly approaches to good death, philosophical and researchbased. The following discussion of good death mirrors those approaches.

\section{Philosophical}

Writers from different disciplines challenged the existence of good death and suggested that cultural ideals of good death imposed behavioral expectations on the dying (Ellison \& Fuller, 1998; Hart, Sainsbury \& Short, 1998, Walters, 2004). Some authors in the health sciences proposed factors that indicated a good death including identification and aggressive treatment of physical, spiritual, psychosocial signs and symptoms for the client and their loved ones (Emanuel \& Emanuel, 1998; Gazelle, 2003). Gazelle (2003) also opined, “Death is rarely good, but a constellation of factors add up to a better death” (p. 95). In describing the conduct of a concept analysis of good death, Welch (2008) suggested that the defining attributes of good death are comfort, journey and loving presence and defined good death as "the inward and outward journey one embarks upon at the end of life in comfort amidst a loving presence” (p.122). 


\section{Research}

Exploration of good death by researchers demonstrated the use of many different methods, both qualitative and quantitative. Some researchers sought to discover participants' perspectives of a good death and gathered data from groups with health care professionals or patients only or mixed groups of patients, professionals and families. All groups exhibited great variety of view about what constituted a good death. Common themes included comfort/pain and symptom management, the presence of someone, preparation/decision making, dignity/affirmation of whole person and not knowing (Hopkinson \& Hallet, 2002; Kim \& Lee, 2003; Pierson, Curtis, \& Patrick, 2002; Steinhauser et al, 2000; Vig, Davenport, \& Pearlman, 2002; Vig \& Pearlman, 2004).

Other researchers used indirect methods to explore good death. A multidisciplinary focus group of eight hospice workers utilized concept mapping to develop a map of the "final journey of life” which included: resolution of emotional, relationship, spiritual levels as well as refocusing on living to dying, transcendence and physical manifestations of the dying process (Forbes \& Rosdahl, 2003). Mak and Clinton (1999), in a literature review, identified seven elements of a good death. The elements included the following: comfort or relief from pain and suffering, opening or being aware of dying, completion or accepting the timing of one's death, control, optimism (keeping hope alive), readiness/preparing for departure, and location/living with one's choice about where to die (Mak \& Clinton, 1999). After the conclusion of a largescale study conducted by the Institute of Medicine, the editors of the report summary noted that too many dying people suffered needless pain and distress because of the many impediments to appropriate care and gaps in science about end of life (Field \& Cassel, 1997). 
Most researchers exploring good death use qualitative methodology. However, some researchers sought to quantify good death by creation of tools. Ternestedt, Andershed, Eriksson \& Johansson (2002) developed a chart audit tool to analyze whether hospice patients experienced a good death according to six "S" criteria: symptom control, social relationships, selfdetermination, self-image, synthesis (patient sums up life) and surrender. Developers of the Quality of Dying Apgar, adapted from the neonatal APGAR score, included pain, non-pain symptoms, advance care planning, peace/dignity and duration as the five main criteria for a good death in renal patients (Cohen, Poppel, Cohn \& Reiter, 2001). Steinhauser et al. (2002) proposed to measure quality of life at the end-of-life with the QUAL-E.

QODD. The quantitative tool with the most use and reliability is the Quality of Dying and Death Instrument (QODD). Researchers at the University of Washington developed and validated the 31-item QODD. Initial validation of the tool took place in Missoula county Montana. After the death of a loved one, family members $(n=205)$ rated the frequency of events in the following domains: symptom and personal care, preparation for death, moment of death, family, treatment preferences and whole person concerns (Curtis et al, 2002, Patrick Curtis, Engleberg, Nielsen \& McCown, 2003). The second part of the tool, then asks that family member to rate how a particular symptom or experience affected the quality of dying (Mularski, Curtis, Osborne, Engelberg \& Ganzini, 2004). The family member also completed six other tools and answered two global rating questions regarding quality of life in the dying person's last seven days and the moment of death. These additional tools and questions allowed the investigator to compare the new tool with established tools. Developers of the tool hypothesized that the higher the QODD score (0-100 points), the higher the quality of death. The mean QODD score was 67.4. Investigators in the initial studies found significant association between 
higher QODD scores and the following variables: death at home $(\mathrm{p}<0.01)$, death in the location the patient desired ( $\mathrm{p}<0.01)$, lower symptom burden ( $\mathrm{p}<0.001)$ (Curtis et al, 2002). The Cronbach alpha was .89, indicating the tool possessed internal consistency. The two global questions significantly correlated with the QODD total score; last seven days $(\mathrm{r}=0.55$; $\mathrm{P}<$ 0.001) and moment of death $(r=0.51 ; \mathrm{P}<0.001)$ (Curtis et al, 2002).

The tool was next tested with family members of enrollees of a two hospices in Washington state ( $\mathrm{n}=96)$. Hospice nurses also completed the instrument. There was no report of statistics on specific items. The authors noted association between higher QODD scores and quality of care $(r=0.41, p=0.002)$, quality of life $(r=0.39, p=0.003)$ and moment of death $(r=$ 0.51, p < 0.001) (Patrick, Curtis, Engelberg, Nielsen \& McCown, 2003).

Refinement of the original QODD resulted in three different adaptations for use in the intensive care unit setting. Mularski, Curtis, Osborne, Engelberg \& Ganzini (2004) explored the agreement among family members of their loved ones quality of death in the ICU while Mularski, Heine, Osborne, Ganzini \& Curtis (2005) examined the quality of dying in the ICU. Data collection for both studies occurred simultaneously. Family members $(n=94)$ of patients who died in four ICU's in two hospitals (n- 38) completed an adapted 23-item ICU-QODD and the original 31-item QODD. In the first report, the investigators found no significant differences in total scores on the two versions of the QODD among family members although families disagreed about the pain control of their loved ones (ICC $=0.26$ ). Dropping the eight items from the original QODD during development of the ICU-QODD was supported as the ICC of the dropped items ranged from 0.01 to 0.36 (Mularski, Curtis et al., 2004). In the second report, the mean ICU-QODD score was 60 on the 1-100 point scale (SD =14) (Mularski, Heine et al., 2005). After multivariate analysis, the investigators noted that four items accounted for the overall 
rating: patient feeling of peace with dying $(\mathrm{p}<0.0001)$, pain under control $(\mathrm{p}<0.0001)$, control of events $(p=0.0066)$, and keeping dignity/self -respect $(p=0.0028)$ (Mularski, Heine et al., 2005). Families rated that their loved ones received adequate pain control only $47 \%$ of the time.

Levy et al. (2005) tested a 21-item ICU-QODD with family members, resident physicians, attending physicians and ICU nurses in two medical ICUs in two hospitals. The health care providers completed the tool within 24 hours of a patient's death and the family member one month after their loved ones death. All four groups evaluated 38 of 91 patient deaths; evaluation of other deaths occurred but not by all four groups. There were statistically significant $(\mathrm{p}<0.001)$ differences in the ICU-QODD total scores among the four groups. Among the health care professionals, nurses $(66.9 \pm 16.3)$ and residents $(67.8 \pm 22.5)$ total scores were the lowest and attending physicians $(81.5 \pm 17.3)$ the highest. Family member $(77.7 \pm 9.3)$ scores fell between the resident and attending physicians (Levy et al., 2005).

The final version of the QODD tested was 14-items. A focus group of nurses selected the items that they believed were pertinent to the ICU environment and that they could evaluate. Nurses who cared for the patient the shift before and at the time of death completed the tool. Additional data collected from the nurses included patient race, withdrawal of life support, performance of cardiopulmonary resuscitation with 8 hours of death, and presence of family at the bedside at the time of death (Hodde, Engelberg, Treece, Steinberg, \& Curtis, 2004). The investigators gathered other demographic data from the medical record. After analysis of 149 completed questionnaires, the mean QODD score was 73.1 (SD 21.4) (Hodde et al., 2004). The tool had high internal consistency with a Cronbach alpha of 0.92. Bivariate analysis showed that total scores were significantly lower with performance of CPR in the last 8 hours of life ( $<<$ 0.01) and significantly higher with presence of someone at the time of death $(\mathrm{p}<0.01)$. The 
investigators also performed a multivariate analysis and found that three variables, someone present (Beta .252, p 0.001), CPR (Beta -.202, p 0.01) and neurology/neurosurgery service (Beta .267, p 0.001) independently predicted the total QODD score (Hodde et al., 2004).

\section{Seminal Reports and Position Statements on Pediatric Palliative and End-of-Life Care}

Provision of good death for children suffering from life-limiting illnesses has been the focus of Children’s Hospice International (CHI), a non-profit organization started in1983 to address the palliative/end-of-life needs of children. The goals of the organization include the following; (a) promote hospice support through pediatric care facilities, (b) encourage the inclusion of children in existing and developing hospice, palliative, and home care programs, and (c) include the hospice perspectives in all areas of pediatric care, education, and the public arenas (CHI, n.d.). This organization was an early voice in the movement to improve pediatric palliative care. Those visionary individuals within CHI are responsible for much of the attention now directed to pediatric palliative/end-of-life care in the literature.

Good death for dying children is the subject of many editorials and position papers found in the literature. Generally, the authors of the editorials and position papers call for improved care for dying children and the families of those children (Feeg, 2005; Feudtner, 2004; Hutton, 2002; Milstein, 2003; Rushton \& Catlin, 2002; Sandler, Kennedy, \& Shapiro, 2004; Stephenson, 2000). Mention of good death is overt in some of the writings and implicit in others. That literature is not in this review.

The National Consensus Project for Quality Palliative Care (NCP) addressed the issue of pediatric palliative care as well. The project is a collaborative effort of a task force of four organizations, American Academy of Hospice and Palliative Medicine, Center to Advance Palliative Care, Hospice and Palliative Nurses Association, and National Hospice and Palliative 
Care Organization. Development of the first and second editions of Clinical Practice Guidelines for Quality Palliative Care resulted from the work of the task force. The guidelines “describe the core precepts and structures of clinical palliative care programs” and provide examples of exemplar palliative care programs (NCP, 2009, p. iv). Discussion of pediatric palliative care occurs in the guideline, but is not the primary or only focus. Given the global focus of the guideline, that work receives mention but not a comprehensive discussion. Instead, this author chose to focus on four seminal works that comprehensively discuss the issue of pediatric palliative and end-of-life care.

Pediatric palliative care and end-of-life care received intense examination at different times from three separate committees or workgroups of the American Academy of Pediatrics (AAP), the National Hospice and Palliative Care Organization (NHPCO) and Institute of Medicine (IOM). Each committee composed and published the results in the form of reports and/or statements. The AAP statement was an obvious inclusion given that advocacy for and the medical care of infants, children and adolescents is the primary focus of pediatricians. Inclusion of a report about pediatric palliative care by the NHPCO, the largest organization representing hospices and palliative care programs and professionals in the United States, was vital given the expertise on end-of-life care. The report by the Institute of Medicine merited inclusion because the comprehensive scope of the report made it the current state of the science about pediatric palliative and end-of-life care.

Additionally, this section includes a review about pediatric palliative care written by Bruce Himelstein, MD and published in the Journal of Palliative Medicine in 2006. Himelstein, a pediatric hematologist/oncologist and program director of palliative care/hospice at Children’s Hospital Wisconsin, writes extensively about pediatric palliative care. Other authors writing 
about pediatric palliative care often cite Himelstein’s writings. His review is a holistic and comprehensive discussion of the subject.

The reports by these three committees and Himelstein's review depict the evolution of pediatric palliative care in the literature in the last decade. These papers represent the best of the current thought about pediatric palliative care. Presentation and discussion of the three committees’ reports and Himelstein’s review comprise the content of this section.

\section{American Academy of Pediatrics}

The American Academy of Pediatrics Committee on Bioethics and Committee on Hospital Care addressed the issue of palliative care for children in 2000 and called for: The development of clinical policies and minimum standards that promote the welfare of infants and children living with life-threatening or terminal conditions and their families, with the goal of providing equitable and effective support for curative, life-prolonging and palliative care (p. 351).

The committee suggested an integrated model of care with the following principles as a foundation: respect for the dignity of patients and families, access to competent and compassionate palliative care, support for the caregivers, improved professional and social support for pediatric palliative care, and continued improvement of pediatric palliative care through research and education (AAP, 2000, p. 351-352). Minimum standards proposed included a consistent medical provider, the availability of expert interdisciplinary care and seamless transitions between care settings, all available around the clock every day.

The committee made seven recommendations. Some recommendations focused on the development of integrated pediatric palliative care programs to provide care for children through the entire course of an illness. Others dealt with professional issues such as education of 
residents and pediatricians, research, changes in regulation of and reimbursement for palliative care and non-support of physician-assisted suicide or euthanasia (AAP, 2000). The AAP never overtly used the word good death in this statement. However, good death is implicit in all the principles and recommendations.

\section{National Hospice and Palliative Care Organization}

The Children’s International Project on Palliative/Hospice Services Administrative/Policy Workgroup of the National Hospice and Palliative Care Organization (NHPCO) issued a report titled A Call for Change: Recommendations to Improve the Care of Children Living with Life-Threatening Conditions in October 2001. In the report, the group discussed the need for services and challenges to meet the end-of-life needs of children. The authors of the report noted the unique challenges of pediatric palliative care, primarily the societal notion that children are not supposed to die (NHPCO, 2001). Other challenges included great variability in survival that interferes with current eligibility criteria for hospice services, varying communication and decision-making capacities given the normal developmental progression of children, the misperception that palliative care begins when curative efforts stop and non-reimbursement for palliative and hospice care (NHPCO, 2001). A significant issue identified was that of current inadequacy of the structures and reimbursement mechanisms of hospices to meet the needs of dying children and their families.

This workgroup made recommendations for clinical, educational and legal/ethical issues as well as research. The most important recommendation pertained to admission criteria, which stated, "the sole admission criteria for pediatric palliative care services must be that the child is not predicted to survive to adulthood” (NHPCO, 2001, p. 4). Other clinical recommendations related to changing reimbursement for care, interdisciplinary care, role and access of families, 
grief counseling for families, schoolmates, teachers, members of community organizations, and professional staff associated with the dying child. Educational issues included the current lack of familiarity with pediatric palliative care and dire need to improve knowledge and skills of professional healthcare providers. Legal/ethical issues covered topics as diverse as extending do not resuscitate orders outside of hospitals to schools and legal provision of decision-making by children. Finally, the workgroup stated that adequate funding of research must occur so that children and families receive appropriate palliative and hospice care rather than care based on data extrapolated from the adult data (NHPCO, 2001).

The NHPCO followed up this work by asking the Children’s Project on Palliative/Hospice Services (ChiPPS) to develop Pediatric Standards (Friebert \& Huff, 2009). The end-result was the first national pediatric practice standards for pediatric hospice and palliative care programs. These standards were first made available in 2009.

\section{Institute of Medicine}

The most comprehensive discussion of pediatric end-of-life care occurred in the 2003 Institute of Medicine (IOM) report When Children Die: Improving Palliative and End-of-Life Care for Children and Their Families. A multidisciplinary committee, The Committee on Palliative and End-of-Life Care, created the report. Patterns and trajectories of childhood death, communication, goal setting, organization and delivery of holistic care from diagnosis through death and bereavement, financing of care, ethical and legal issues, provider education, and research comprise the topics found in the report. The report authors also discuss and define good death. The following seven working principles guided the committee’s work: 
1. Appropriate care for children with life-threatening medical conditions and their families is designed to fit each child's physical, cognitive, emotional, and spiritual level of development.

2. Good care involves and respects both the child and the family.

3. Families are part of the team.

4. Effective and compassionate care for children with life threatening conditions and for the families is an integral and important part of care from diagnosis through death and bereavement.

5. Professionals caring for children have special responsibilities for educating themselves and others about the identification, management, and discussion of the last phase of a child's fatal medical problem.

6. Both individual change and organizational change are needed to provide consistently excellent palliative, end-of-life and bereavement care for children and their families.

7. More and better research is needed to increase our understanding of clinical, cultural, organizational, and other practices or perspectives that can improve palliative, end-of-life, and bereavement care for children and families (Field \& Behrman, 2003, p. 29).

This report was more than an accounting of what happened or did not happen in end-oflife care of children in America. The committee made several recommendations in the report, which applied to the organization and provision of palliative and end-of-life care. Specifically, the authors suggested the development and implementation of developmentally appropriate and culturally sensitive clinical practice guidelines and protocols to meet the needs of children and 
their families. Clinical recommendations included the following: information about disease prognosis and treatment, early discussion about goals and preferences about care, effective treatment and diagnosis of physical and psychosocial symptoms, decision-making at the end-oflife and provision of bereavement services. All recommendations included the need for collaboration among many groups such as health care providers, children's hospitals, hospices, community health agencies, government agencies and others to develop and implement the recommended guidelines and protocols. Other recommendations in the report applied to improvements in the reimbursement and eligibility for hospice care and education of health professionals about pediatric palliative and end-of-life care. The final group of recommendations suggested directions for research since the most common phrases in the report were "Research is limited” or “systematic data are not available” (Field \& Behrman, 2003).

\section{Himelstein}

Himelstein (2006) proposed that pediatric palliative care was more than care for the dying but rather "helping children and families to live to their fullest while facing complex medical conditions” (p. 163). He viewed palliative care as different from hospice care in that palliative care occurred earlier in a child's disease process, often at diagnosis. Palliative care was viewed as appropriate for children with conditions for which (a) cure or life-prolonging treatment is available but may fail, (b) long periods of intensive treatment aim to prolong quality of life, (c) treatment from diagnosis is purely palliative, and (d) severe, non-progressive disability leads to increased vulnerability to health complications (Himelstein, 2006, p. 163). In the review, Himelstein (2006) delineated the following fifteen principles of pediatric palliative care:

1. Care is child-focused, family-oriented, and relationship-centered. 
2. Care focuses on relief of suffering and enhancing quality of life for the child and family.

3. All children suffering from chronic life-threatening and terminal illnesses are eligible.

4. Care is provided for the child as a unique individual and the family as a functional unit.

5. Palliative care is incorporated into the mainstream of medical care regardless of the curative intent of therapy.

6. Care is not directed at shortening life

7. Care is coordinated across sites of care delivery.

8. Care is goal directed and is consistent with the beliefs and values of the child and his or her caregivers.

9. An interdisciplinary team is always available to families to provide continuity.

10. Advocacy for participation of the child and caregivers in decision-making is paramount.

11. Facilitation and documentation of communication are critical tasks of the team.

12. Respite care and support are essential for families and caregivers.

13. Bereavement care should be provided for as long as needed.

14. Do not resuscitate orders should not be required.

15. Prognosis for short-term survival is not required (p. 163).

Specific discussion of pain and symptom management including medications and dosage ranges made this review unique from the previous three.

Discussion of the development of spirituality and of the concept of death in children was another aspect of the paper. Himelstein (2006) reminded the reader that all children, even those 
with life-threatening illnesses, are developing human beings in body, mind and spirit. Children’s spirituality and understanding of death develop over time. Consideration of the child's understanding of death and support of the developing spirituality are therefore critical elements of care for the child with a life-threatening illness. Himelstein did not overtly state that good death was the goal. Again, good death is implicit in the guidelines and discussion of physical and psycho-spiritual care.

\section{Neonatal and Infant Palliative and End-of-Life Care}

The focus on neonatal and infant palliative and end-of-life care, as pediatric palliative care occurred only recently in the literature. Few authors tackled the challenging topic prior to 2000. In 1973, Duff \& Campbell were among the first to suggest provision of comfort care for dying newborns. Whitfield, et al (1982) elaborated on the concept of comfort care for dying newborns by suggesting the application of hospice concepts to care in to the NICU population. Delivery of “comfort care” occurred in a special room near the NICU.

Mangurten (1990) noted that while comfort care was positive for families, the NICU was not home. He suggested that parents have the option of home centered care and presented case studies of six families who cared for a dying baby at home. These case studies illustrated the advantages and challenges of home care. The primary advantage of home care of the dying neonate was that parents were the primary caregivers, not professionals. Home care also allowed for privacy, support of the family by extended family and friends, more personalized care and avoidance of hospital rules, protocols and costs. Challenges of providing care at home included ambivalence of parents once home, the needs of siblings and the mechanics of how and where to take the infant's body after death. Upon evaluation of the experience, families made the following suggestions: provision of more active support for the mother, specific written 
instructions, the availability of parent-parent support and education about the possibility of organ donation (Mangurten, 1990, p. 306). All of the families reported no regret on the decision for home care.

Since those early papers, others have published more in-depth examinations of neonatal palliative care. Toce (2000) focused on the challenges of decision-making in withdrawal and withholding of treatment for high-risk newborn given the sometime uncertain prognosis of some conditions. Modification of the NICU environments to make them more family and neonate friendly and provision of bereavement and grief support for both the families and staff were suggestions made by Toce.

The very premature and/or dying neonate may only exhibit pain through physiologic variables and not behaviorally as do older infants (Walden, Sudia-Robinson \& Carrier, 2001). Therefore, it is vital that health care professionals working with dying neonates in the NICU receive education and possess competencies in stress and pain assessment and management specific for the neonate. Walden, et al. (2001) discussed physical, environmental and social comfort strategies that included opioids, reduction of noxious stimuli from invasive procedures, positioning, attention to light and sound levels, warmth and touch.

There was general agreement among authors about the necessary basic components of neonatal palliative and end-of-life care. Those basic components included comfort care, decision-making and bereavement support (Caitlin \& Carter, 2002; Carter \& Bhatia, 2001; Craig \& Goldman, 2003; Gale \& Brooks, 2006; Lisle-Porter \& Podruchny, 2009; NANN, 2010; Romesberg, 2007; Toce, 2000; Walden, Sudia-Robinson \& Carrier, 2001). Despite those general agreements, controversies and challenges surround the topic. Romesberg (2007) noted the following challenges: (a) financing of palliative care programs, (b) the logistics of providing 
palliative care to neonates transported from a distant site, (c) language and cultural differences between parents and health care professionals, (d) care and support of grieving health care providers, and (e) lack of education of health care providers about palliative and end-of-life care. Other barriers to provision of palliative to neonates/infants includes inadequate staffing, an unconducive environment, technological imperatives, parental expectations/demands, moral distress and lack of education as barriers to effective end-of-life care (Kain, 2011; Wright, Prasun, \& Hilgenberg, 2011).

Another controversy discussed in the literature is the question of who receives neonatal palliative care. Bhatia (2006) suggested consideration of fetal or neonatal palliative care with the occurrence of the one of three following categories of neonate: (a) neonates at the limit of viability, (b) neonates with lethal congenital anomalies, and (c) neonates with serious medical or surgical conditions not responding to maximal therapy or for whom continued medical treatment prolongs suffering (p. S24). However, not addressed in the article was when to initiate palliative care. This oversight has implications for the intensity of resuscitation efforts in the delivery room or lack thereof. Protocols, like those used to guide resuscitation, may assist health care professionals know when and how much to intervene.

\section{Neonatal Palliative Care Protocols and Programs}

For some health care professionals, protocols provide for delivery of consistent and effective palliative care and a good death for neonates and infants. Carter and Bhatia (2001) described the multidisciplinary process of creating and implementing a comfort/palliative care guideline for neonatal practice in one academic medical center. The protocol provided guidelines for delivery of care to three specific neonatal populations: those with extreme prematurity, overwhelming illness and lethal anomalies. The authors cautioned that this protocol 
was only a step and that implementation required the consideration of five sets of interests including that of the patient, family, staff, institution and society (Carter \& Bhatia, 2001). The Neonatal End-of-Life Palliative Care Protocol is another example of a protocol. Over an 18-month period, a 101-member panel from 93 locations in the United States and 4 from abroad used Delphi research method to create the protocol (Caitlin \& Carter, 2002). The panel utilized 16 published and non-published end-of-life protocols developed by various professional and lay groups as well as literature and clinical experience. The comprehensive protocol addressed the following issues:

1. Planning for a palliative care environment

2. Prenatal discussion of palliative care (physician considerations and family considerations)

3. Transport issues

4. Which newborns should receive palliative care

5. Introducing the palliative care model to parents

6. Optimal environment for neonatal death

7. Location for provision of palliative care

8. Ventilator removal, pain and symptom management (preparation and process)

9. When death does not occur after cessation of aggressive support

10. Discussion of organ and tissue procurement and autopsy

11. Family care: cultural, spiritual and practical family needs

12. Family follow-up care

13. Ongoing staff support (Caitlin \& Carter, 2002, p.185-195). 
After its release in 2002, the Neonatal End-of-Life Palliative Care Protocol quickly influenced neonatal palliative care. Gale \& Brooks (2006) described the process of planning, development, and implementation of a palliative care program in a 55 bed NICU using the protocol as a resource. A planning committee and subgroups within the committee created a Palliative Care Protocol and a NICU Palliative Care Pathway, designed to be a resource for the bedside nurse, as well as a Palliative Pain Management Resource. The pathway, since modified to a checklist for ease of use, named seven phases of palliative care. The phases follow the neonate and family from the time when an uncertain outcome becomes evident to introduction and initiation of palliative care through death and dying and finally, bereavement. The pathway specified the observations, expected outcomes, family discussions and consultations, activity and safety, nutrition, medications, tests/treatments, and support services that would occur during each phase (Gale \& Brooks, 2006).

Lisle-Porter and Podruchny (2009) wrote about family-centered end-of-life care for the dying neonate. The authors included a guideline for end-of-life care of the neonate. The guideline was divided into pre-death, active dying and after death activities and included specific suggestions regarding communication, environment, pain management, and parental activities (Lisle-Porter \& Podruchny, 2009). Provision of culturally and religiously competent care was specifically emphasized by these authors.

\section{Home Care of the Dying Infant}

Most of the protocols discussed care based on the assumption that most neonatal deaths occurred in the NICU. Cavaliere (2007), like many others, noted that these units were not the ideal setting for delivery of palliative care and a good death despite efforts that made the environment more family-centered. The protocols all included provisions to take the neonate 
home to die if the parents were willing and able. Going home required more than a willing spirit and involved much planning and coordination of care. Parents needed information about what to expect when the neonate is actively dying such as commonly seen symptoms, management of those symptoms by medications or alternative methods, how to draw up and give the meds, and perhaps most importantly 24 hour access to support (Craig \& Goldman, 2003). Many of the authors suggested the use of home health or hospice services with experience in caring for dying infants or ideally pediatric hospices but noted this could be a significant challenge in some areas of the country or world (Caitlin \& Carter, 2002; Craig \& Goldman, Gale \& Brooks, 2006). Caitlin and Carter (2002) opined the need to work with local hospices to meet the needs of dying infants, for without the challenge to do so, pediatric hospice or home health services would not evolve.

\section{Position Papers}

Within the last three years, two groups actively involved in the care of neonates and infants developed and published position papers/statements regarding palliative care for neonates and infants. In 2009, The National Perinatal Association (NPA) (2009) published a position paper that stated the following:

Despite medical advances in diagnostics, treatments, and technology, mortality continues to exist in the perinatal field. Infants are born too early and with congenital conditions that are incompatible with meaningful life. These children deserve the best end-of-life care that health care providers can provide. The National Perinatal Association (NPA) recognizes that hospice and palliative care services are often 
offered very late in the trajectory of adult illness and, for infants and children, are often not offered at all (para 1).

Topics addressed in the position paper include neonatal palliative care, perinatal hospice, and strategies for training health care professionals.

The next year the National Association of Neonatal Nurses (NANN) published a position statement regarding palliative care. The position of NANN (2010) is as follows: "Palliative and end-of-life care for infants and their families are an integral component of neonatal care, and neonatal nurses should be trained and participate in providing services involved in this essential care” (para. 3). Several recommendations regarding provision and organization of palliative care follow in the paper and include: (a) when palliative care should be offered, (b) support for decision making with prenatal diagnosis, (c) educational material for parents, (d) assertion of the parents' role as active participants in decision-making, (e) provision of family support services, (f) focus on comfort care, (g) need for NICU's to work with local hospices or palliative care organizations for a seamless continuity of care, (h) end-of-life care, (i) provisions for parent support with removal of life-sustaining technology, (j) bereavement support after death and (k) support services for all member of the healthcare team (NANN, 2010).

\section{Palliative Care for Neonates/Infants with Life-Threatening Congenital Anomalies}

Almost none of the literature about palliative and end-of-life care for neonates and infants focused on neonates with life-threatening congenital anomalies. Leuthner (2004) focused specifically on the provision of palliative care for infants with lethal anomalies. Palliative care is an option whether the diagnosis of a life-threatening congenital anomaly occurred during the prenatal period or post-delivery. Prenatal diagnosis of a lethal anomaly allows for the application of perinatal palliative care or hospice options and for discussion of decisions around 
delivery: timing, type of delivery, location whether at a local community hospital or a tertiary center and aggressive resuscitation of the neonate (Leuthner, 2004). Recommendations regarding the surprise delivery of a neonate with anomalies included resuscitation and stabilization in the NICU until further evaluation allowed for definitive diagnosis unless the diagnosis was obvious in the delivery room. Components of palliative care and a good death provided for that all previously presented: advanced care planning, an appropriate environment, and holistic care for the neonate and family that included bereavement care. The author noted that home care was a very real option for some families given the variable life span with some anomalies. However, it was vital that any plan for discharge to home included home health or hospice support (Leuthner, 2004). Leuthner and Jones (2007) followed up this article with another that discussed a perinatal palliative care.

Perinatal Palliative Care. Perinatal palliative care starts during the pregnancy upon diagnosis of a life-limiting congenital anomaly in the fetus or after birth with the diagnosis of a life-limiting anomaly in the newborn (Bhatia, 2006; Breeze, 2007; Engelder, Davies, Zeilinger, \& Rutledge, 2012; Leuthner \& Jones, 2007; Sumner, Kavanaugh, \& Moro, 2006). This care continues during the lifespan of the fetus/baby and through bereavement. This model of care is also known as perinatal hospice. Current literature still mostly discusses the concepts of or structure of perinatal palliative/ hospice care. Little research exists about this newer model of care.

\section{Parents}

Parents are vital participants in any discussion regarding neonatal or infant palliative or hospice care. All of the previously presented literature included a discussion of parents as active participants in the process of caring for dying neonates and infants to enable a good death. 
Workman (2001) wrote of her personal experience in caring for families of dying infants and their families. She shared examples of how to present information, such as describing the respiratory sounds made by the dying baby as "sighing” versus "struggling to breathe" (Workman, 2001, p. 571). Offering the family options, such as giving the baby a bath or being present for extubation, were also a part of the process of helping families cope. Preparation and ceremony, easing fear, withdrawing life support, and letting go were the organizing principles of the article (Workman, 2001).

Parental grief and nursing interventions to support parents after stillbirth or newborn death in the hospital was the subject of an article by Kavanaugh and Moro (2006). The authors stressed that nurses should be knowledgeable about the rights of infants and of parents when an infant dies. Additionally, they suggested that nurses be familiar with the middle range theory of caring by Swanson (Kavanaugh \& Moro, 2006). Interventions were organized under the categories of being present, helping parents create cherished memories, guiding parents in decisions about autopsy/burial, and follow-up visits and phone calls (Kavanaugh \& Moro, 2006).

Romesberg (2004) discussed grief as a component of neonatal palliative care and implications for practice. Suggested interventions for nurses included a) encouraging parents to hold and name the baby, b) assist in creation of mementos such photos and footprints, c) provision of education and support through decisions to withdraw life support, d) consideration of family's spiritual and cultural traditions, and e) referral to peer support groups or professional counseling (Romesberg, 2004). Also discussed was the need for research and education of health care professionals regarding palliative and end-of-life care. 


\section{Review of the Research}

\section{Neonatal and Infant Palliative and End-of-Life Care}

Much of the literature about palliative care for the neonatal and infant population was in the form of clinical descriptions of, protocols for, and position statements about palliative care. It was a challenge to find published research on neonatal and infant palliative care to determine the evidence upon which the various authors based the various descriptions and protocols. Use of various combinations of the terms neonate, infant, end-of-life, infant death, death, end-of-life, hospice, and palliative care guided the search in Academic Search Premier, Medline, CINAHL, Health Source/Nursing Academic Edition and Ovid databases.

Inclusion of a published study occurred if the conduct of the research was in the United States. Research that originated from any other countries merited exclusion because of the differences in the medical culture and the option of legal euthanasia in some. Also excluded were articles that focused on health care providers with topics such as (a) palliative care education, (b) issues of comfort in provision of palliative care or (c) stress related to caring for dying neonates and infants. For inclusion, the study had to focus on infants who died of something other than stillbirth, Sudden Infant Death Syndrome (SIDS), prematurity or consequences of untoward perinatal events. Death of infants with the excluded conditions listed above typically occurred in an unexpected manner and there was limited time or opportunity for parents to experience being parents. The focus of this study is on infants for whom death was an expected future event, for whom palliative and hospice care were real options and whose parents had some time to experience being parents, even though that time was potentially brief.

Few published studies focused on palliative care for neonates or infants. This mirrored the findings of a descriptive integrative literature review conducted by Moro, Kavanaugh, 
Okuno-Jones \& VanKleef (2006). The investigators found only ten studies that met very strict inclusion criteria. Practices of withdrawing or withholding life-sustaining treatment, pain management during ventilator withdrawal, parents and the decision-making process and the dying process were the categories under which the authors organized the discussion.

Much of the research about end-of-life care of neonates explored decision-making around withholding or withdrawing life support. Sometimes decisions about withholding life-saving interventions occurred during the birth process. Other difficult decisions included withdrawal of aggressive treatment, particularly withdrawal of ventilator support (Abe, Catlin \& Mihara, 2001; Tan, Totapally, Torbati \& Wolfsdorf, 2006; Wall \& Partridge, 1997). Few studies focused on the provision of palliative care services to neonates. The published research was of a descriptive nature, which was appropriate given the recent emergence of this area of study.

Withdrawal and withholding of life support. Withdrawal and withholding of life support received attention in the literature. Most research relative to the topic used retrospective chart audits to examine medical records for frequency and documentation of withdrawal of life support. Researchers at various sites found that end-of-life decisions in NICU's involved ventilator withdrawal approximately $70 \%$ of the time (Wall \& Partridge, 1997). Ventilator withdrawal did not always include the writing of do not resuscitate (DNR) orders. Wall \& Partridge (1997) found only 22\% of charts and Abe et al (2006) found no charts with a DNR order. A topic related to ventilator withdrawal was the use of opioids to ease symptoms of distress. Abe et al (2006) noted that lowest use of opioids occurred in the NICU, and only about half of infants removed from the ventilator in the Wall and Partridge (1997) study received opioids after extubation. 
Palliative care. Few studies focused on palliative care for neonates and infants. Pierucci, Kirby and Leuthner (2001) conducted a retrospective chart audit to describe end-of-life care of infants less than one year of age at a children’s hospital between 1994 and 1997. Charts selected for audit were those of infants, less than a year of age, who died at Children's Hospital of Wisconsin during the 4-year period. The sample consisted of the charts of 196 infant that died. Only 25 (13\%) of the sample received a palliative care consult. Palliative care consult infants spent fewer days in the intensive care unit $(6+/-8$ vs. $15+/-31, \mathrm{p}=.002)$, even with matching and controlling for diagnosis (6 +/- 8 vs. $14+/-28, \mathrm{p}=.01)$ than non-consult infants (Pierucci, Kirby \& Leuthner, 2001). The infants, whose families received palliative care consult, died significantly less often, proportionally, in the NICU or PICU than did infants without a palliative care consult $(68 \%$ vs. $97 \%, \mathrm{p}<.001)$. Dying infants whose families had a palliative care consult experienced significantly fewer invasive procedures such as central line (64\% vs. $92 \%, \mathrm{p}<.001)$, feeding tube (64\% vs. $95 \%$, $\mathrm{p}<.001$ ) or endotracheal tube ( $60 \%$ vs. $94 \%$, $\mathrm{p}<.001)$ insertion or lab draws ( $56 \%$ vs. $94 \%, \mathrm{p}<.001$ ) in the last 48 hours of life than non-consult infants. There were no statistically significant differences in CPR attempts or withdrawal of mechanical ventilation between the two groups. However, there were significant differences between consult and non-consult infants regarding the withholding of mechanical ventilation. Seven (28\%) of the consult infants had mechanical ventilation withheld versus 6 (4\%) of the nonconsult group $(\mathrm{p}<.001)$. The medical records of the recipients of a palliative care consult contained more documentation of the family's emotional status ( $92 \%$ vs. $70 \%, \mathrm{p}<.05)$ than those families without a consult. Referrals to a chaplain ( $64 \%$ vs. $34 \%$, p <.001) or social services $(80 \%$ vs. $49 \%, \mathrm{p}<.001)$ occurred more in the palliative care consult group. The findings suggest that palliative care consultation positively affected the end-of-life care of 
infants. The authors also noted palliative care consultation was an underutilized service given that only 13\% received a consult (Pierucci et al. 2001).

Two members of the above team conducted a retrospective chart audit, but this time to explore the services provided to infants and their families with a palliative care consult. Fiftyone infants died during the period of the study, January of 1996 to June of 1998 (Leuthner \& Pierucci, 2001). Only 12 (23.5\%) had palliative care consults. Most of the consult infants $(n=10)$ died within two weeks of consult although a one infant lived up 7 months of age. The most common reason for consult was for home hospice services, although discussion of withdrawal of life support or nutrition/fluids was common. Only four of the consult infants died at home. Another four died in private rooms out of the intensive care unit after the palliative care team suggested transfer. Advance directive planning, environment, comfort measures and family support were the categories of services provided to the dying infant and families. The authors also found that not all families wanted a home death (Leuthner \& Pierucci, 2001).

Leuthner, Boldt and Kirby (2004) conducted a retrospective audit of Wisconsin death certificates to determine the setting in which infants died. The study also explored outpatient services available to dying infants by hospices or home health agencies by survey. The sample included infants who died from congenital anomalies $(n=508)$ or conditions of the perinatal period $(n=1,030)$. This group accounted for $60 \%(n=1,538)$ of all infant deaths for the years 1992 to 1996. Only four percent $(n=62)$ of these infants died at home. The authors also surveyed all hospice and home health agencies for provision of services to pediatric clients. Thirty-seven of 91(40\%) hospice or home health agencies in the state reported being able to care for dying children, yet only 20 had provided that care. Most of the agencies $(n=17)$ had only cared for one or two dying infants. The authors also discussed barriers to provision of palliative 
and end-of-life care for infants at the agency or physician level. The primary community agency barrier is the inability and lack of experience in provision of care to for dying infants and children. Distance between the agency and family's home can also be a barrier in rural area. The hospice admission criteria requiring a terminal or less than 6 months to live, which does not allow for palliative care at home as curative efforts continue is a major barrier. Physician barriers arise from lack of awareness of community services, valuing their own care over community agencies, and a lack of accurate prognostication (Leuthner, et al., 2004).

\section{Parents and Neonatal and Infant Palliative Care}

The examination of neonatal and infant palliative care must include the research specific to parents and neonatal and infant palliative care given the family-centered approach of neonatal and pediatric care. That family-centered approach makes it almost impossible to separate the parents' experience from that of the infant. Parents are an essential element for a good death of an infant. Additionally all the protocols assume active and interested parents.

As with the literature on palliative care for neonates and infants, it was a difficult to find published research that met the inclusion criteria. This author used the same search criteria as above with the addition of the word parent, mother or father. Parents' experience of loss of a baby through miscarriage, stillbirth and neonatal death was a topic commonly researched. As previously noted, those studies merited exclusion because death was unexpected which allowed parents limited time or opportunity to be parents. Given the inclusion criteria, only three published study merited inclusion into this section of the review of the research.

\section{Parents who experienced the death of an infant to lethal anomalies. Congenital}

anomalies are the primary cause of death in infants, yet very few researchers specifically study this group of neonates, infants and parents. The investigator found three research studies that 
met the search criteria. A systematic review was not included in this review as some of the studies included in the review were from international sources.

Study One. One group of researchers, in a mixed method study, examined the parents' experience of the death of an infant with a life-threatening condition of the perinatal period or congenital anomalies (Brosig, Pierucci, Kupst \& Leuthner, 2007). Bereaved parents (n=19) completed the Revised Grief Experience Inventory (RGEI) and a semi-structured interview 6 months to 2 years after the death of their less than 1-year-old infant. Scores for the RGEI were significantly lower for this group of parents $(\mathrm{P}<0.0001)$ than normative scores for parents who lost a child (Brosig et al., 2007). The researchers found no differences in grief scores between parents who used palliative or hospice care and those who did not.

Analysis of the taped interviews followed using The Post-Death Adaptation Scale (PDAS) to rate coping. Scores for this group of parents, when compared to normative scores, indicated positive adaptation ( $\mathrm{t}-5.4, \mathrm{p}<0.0001)$. Common themes identified from the semistructured interviews highlighted the importance of honesty, empowerment to make decisions, care of them (the parents), provision of an emotionally and physically supportive environment, sensitive and professional nursing care, involvement of the physician and support from others health care providers (Brosig et al., 2007).

Bereaved parents also discussed strategies that assisted in coping with their infant's death. Strategies which bereaved parents used included seeking support from family and/or a bereavement support groups, focusing on the surviving children, memorializing the dead infant in some manner, turning to God, and "giving back" by monetary donations or volunteering personal time to help other children (Brosig, et al., 2007). The investigators asked parents what could make the end-of-life experience better for other families. The bereaved families 
recommended that parents ask questions and stay informed, stand by their decisions, involve the other children, and participate in the baby's care as much as possible (Brosig et al.).

Examination of the demographic data showed that 18 of the 19 infants (95\%) died in the hospital, only one died at home. Seventy-six percent of the infants who died in the hospital, died in either a NICU or PICU. Hospice and/or palliative care team involvement occurred in $42 \%$ of the deaths ( $n=8)$ (Brosig, et al., 2007). These data were congruent with findings from other studies with the exception of hospice/palliative care team involvement. The percentage of dying infants who had hospice or palliative care team involvement was higher in this study than any previous study. One must note that the investigators of this study are some of the same group, who conducted the studies that examined the impact of palliative care consultation on the care of dying infants. The higher numbers in this study indicate increased utilization of palliative care consultation in this one institution, Children’s Hospital of Wisconsin. The investigators discussed several limitations of the study including the notion that parents who were coping better with the death of their infant were more likely to volunteer to participate in the study. The authors noted the need to improve end-of-life care for infants and their families (Brosig et al.).

Study Two. Another study that merited inclusion used a longitudinal, qualitative, descriptive design to explore the process of parental bereavement (Tan, Docherty, Barfield \& Brandon, 2011). This study was a secondary analysis of data collected for a study about parent and health care provider decision making for infants with complex life-threatening conditions. The investigators conducted open-ended interviews with 14 parents of 8 infants with complex life-threatening conditions. Interviews took place monthly while the infant was alive and until death. Additional interviews took place one week after the occurrence of a life-threatening event and at six weeks and six months after the death of the infant. 
The researchers used content analysis to analyze field notes and transcribed interviews. The findings were organized into the following categories: (a) having expectations, (b) continuity of care, (c) memory making, (d) wide network of support, and (e) altruism (Tan et al., 2011). Within the categories, the researchers delineated themes.

Themes of having expectations were entertaining possible outcomes, decisions they can live with, and speaking with other families (Tan et al. 2011). Continuity of care themes included having a personal witness and provider care. Remembering time spent together and physical memory making comprised the themes of memory making. A wide network of support themes were support from strangers and affirmation of their strength and decisions made. Finally the themes of altruism were helping others through their experience and passing on personal items (Tan et al., 2011).

The researchers provided no data on the site of death or the use of palliative or hospice care. Therefore it is unknown if at the end of life the infants received palliative or hospice care. A limitation that the researchers note is related to the fact that the original study was not about bereavement. Different questions would have been asked had the researchers been specifically exploring parental bereavement rather than decision making (Tan et al, 2011).

Study Three. This third study explored the experiences of bereaved women who had continued a pregnancy after diagnosis of a lethal fetal anomaly (Lathrop \&VandeVusse, 2011). The 15 participants were recruited from a perinatal bereavement support agency. Interviews took place 1-12 years after the death of the baby.

Narrative analysis was the method used to analyze transcripts of interviews. Lathrop and VandeVusse, 2011) noted that “the element of time was prevalent in the mothers' stories” (p. 24). Themes arose out of the connection between the mothers' stories and the passage of time. 
Therefore, continuity, evolving changes, transient phases, and connecting past to present comprise the themes (Lathrop \& VandeVusse, 2011).

Continuity refers to the feelings and thoughts still present despite the passage of time (Lathrop \& VandeVusse, 2011). Feeling of love and connection for the baby, the pain of loss, and a sense of the baby's ongoing presence in their lives were major elements of the theme of continuity. Another element of continuity was renewed grieving on significant days such as the day of diagnosis, birth or death of the baby.

The next theme is of evolving changes. Lathrop and VandeVusse (2011) point out that the theme of evolving changes theme is in contrast to the first theme of continuity. This theme indicates that the intensity of many of the feelings and thoughts of the mothers changed over time. Reflections about personal changes and growth were also an element of the theme of evolving changes (Lathrop \& VandeVusse, 2011).

Transient phases characterized transient periods of time when certain feelings or events were predominant (Lathrop \& VandeVusse, 2011). Mothers shared feeling numb at specific points of time such as time of diagnosis. The time around the baby's death was the most difficult phase. Some mothers described certain points of time as being on a roller coaster with alternating emotional highs and lows.

Finally, connecting past to future refers to looking at the transition from then to now (Lathrop \& VandeVusse, 2011). Many of the mothers looked for life lessons from the experience of loss. Some reported a lasting emotion such a pride or gratitude. Lathrop and VandeVusse (2011) surmised that mothers create continuity between a painful past and their ongoing lives. 


\section{Conclusions}

Good death provides the conceptual foundation for the published literature demanding improved end-of-life care for infants, children and adolescents. Good death is the goal for health care professionals caring for dying individuals and a valid area of scholarly inquiry. The recent focus on palliative and hospice care for neonates and infants in general and specifically for those with congenital anomalies is necessary and long overdue.

Given the relative "newness" of the topic, much of the literature relative to pediatric palliative and end-of-life care is still in the form of position papers, editorials and reviews. The seminal reports and position statements provide readers with the state of the science for good death for pediatric clients and their families and are a good first step to raise awareness. Members of the various workgroups and committees wrote clinical, educational and policy recommendations to improve pediatric palliative and end-of-life care and achieve good death. Additionally, all the authors of the reports noted the need for "more and better" research in order to provide care based on evidence.

The literature that specifically explores palliative and end-of-life care for neonates, infant and their families is in beginning stages. Based on personal professional experience, some authors of that literature anecdotally shared tips how to care for dying infants and their families. Others proposed extensive protocols to guide care, again from professional experience. However, given the paucity of research, the evidence these authors base these informal suggestions and formal protocols for care is limited. Even the authors of the IOM report note that "Research is limited" or "systematic data are not available” (Field \& Behrman, 2003). Research that explores palliative and end-of-life care for neonates and infants is scant. The process of ventilator withdrawal and withholding is a common subject. Palliative care for 
infants was the subject of research in three different studies by one group from Children's Hospital of Wisconsin. Despite the fact that anomalies are the primary cause of death in the first year of life, only three groups of researchers explored the experience of parents whose infant died of a congenital anomaly. There are significant gaps in the knowledge of palliative and endof-life care for neonates and infants. The published literature about the topic generally arises from professional experience, not empirical evidence. Very little research evidence exists upon which to base appropriate and effective end-of-life care for these vulnerable patients and their families so that a good death is possible. The conducted studies all shared the limitations of small sample size, use of death certificates, and limited geography. The two studies that specifically examined parents whose infant with life-limiting congenital anomalies died had the above limitations in addition to possible selection bias.

Much work needs to take place so evidence exists to provide dying infants and their families with a good death. This author explored the experience of parenting an infant with a life-limiting congenital anomaly, seeking to understand more about the process of becoming and being a parent while caring for an infant who will most likely die in the first year of life. This study adds to the limited body of knowledge by creation of a substantive grounded theory to describe the parents' experience of parenting an infant who died of a life-limiting congenital anomaly. Knowledge gained from this study assists in provision of good death for future babies with life-limiting congenital anomalies and their families. 


\section{CHAPTER THREE: METHODS}

Infants dying from life-limiting congenital anomalies and their families suffer from inadequate access to optimal end-of-life care at great physical, psychological and economic cost. These costs are borne by the infants, their families, their health care professionals and ultimately all of society. There is scant research to assist health care professionals in delivering that vitally needed end-of-life care. Therefore, it is vital that more researchers examine this area of health care.

Qualitative research methods are appropriate when scant information exists about a phenomenon. Lobo (2005) proposes that qualitative research is the bench science of nursing and provides foundational knowledge for the subsequent development of nursing interventions specific to a phenomenon. Classic grounded theory is a research methodology that many researchers view as qualitative. Glaser (1998), one of the discoverers of grounded theory, disputes this notion and instead sees grounded theory as applicable for any type of data. Nevertheless, classic grounded theory is a powerful method to understand unfolding processes, such as parenting a dying infant.

The dearth of literature specific to parenting infants with life-limiting congenital anomalies supports the use of qualitative methods. How can nursing develop appropriate interventions when so little is known about the experience of these parents? Marcellus (2005) suggests that the knowledge gained from grounded theory can potentially assist maternal-child nurses in the search to discern the needs of mothers, fathers, infants and their families. Classic grounded theory is an appropriate method to explore the process parents undergo as they experience the birth, life and death of their infant from a life-limiting congenital anomaly. 
The purpose of this study was to: (a) explore the process of parenting as parents experience the birth, life and death of an infant with life-limiting congenital anomalies and (b) to develop a substantive theory to describe the process of parenting from birth to death. Ultimately, the theory will help guide nursing practice. In this chapter, the author will discuss the roots of classic grounded theory and the method itself. Also described in this chapter is the conduct of this study including sample size, inclusion criteria, recruitment of participants and specifics relevant to the interviewing process.

\section{Classic Grounded Theory}

Classic grounded theory as a method of research emerged from sociology in the late 1960’s. It was revolutionary. Systematically generated theory arising from systematically gathered data challenged the traditional model of using data to verify theory. A more thorough discussion of the roots of grounded theory follows later in this chapter. Today, many disciplines in many countries accept grounded theory as a method of inquiry.

The outcome of a grounded theory study is not findings, but rather a substantive theory. A substantive theory is an "integrated set of conceptual hypotheses that account for much of the behavior seen in a substantive area” (Glaser, 1998, p 3). For example, in this study the goal was a substantive theory to account for the behavior of parents as they parent their infant with a lifelimiting congenital anomaly across the infant’s limited lifespan.

Two other concepts are of central importance to grounded theory: theoretical sensitivity and constant comparison (Glaser, 1978; Marcellus, 2005; McCann \& Clark, 2003). Theoretical sensitivity refers to the openness required of the researcher. When entering the field the researcher needs to have few if any preconceived ideas, primarily no hypothesis (Glaser). 
Constant comparison is the simultaneous collection and analysis of data. This allows the researcher to go between the data and analysis to generate a theory.

\section{Roots of Grounded Theory}

Barney Glaser and Anselm Strauss discovered classic grounded theory in the late 1960’s during the conduct of research on dying in hospitals (Glaser, 1978). The collaborators wrote about their discovery in The Discovery of Grounded Theory in 1967. At that time, classic grounded theory was revolutionary as it challenged the prevailing view that quantitative methods were superior (Charmaz, 2000). In 1995, Charmaz (as cited in Charmaz, 2000) wrote the new method specifically challenged

a) the arbitrary divisions between theory and research, b) views that qualitative research served as a precursor to more rigorous quantitative methods, c) claims that the quest for rigor made qualitative research illegitimate, d) beliefs that qualitative methods are impressionistic and unsystematic, e) separation of data collection and analysis, and f) assumptions that qualitative research could produce only descriptive case studies rather than theory development. (p. 511)

Glaser and Strauss came from diverse academic backgrounds but shared a common discipline: sociology. Glaser studied quantitative methodology and qualitative math with Paul Lazarfeld at Columbia University, explication de text at the University of Paris and theory construction with Robert Merton also at Columbia (Glaser, 1998). Strauss’s mentors were Herbert Blumer and Everett Hughes at the University of Chicago, who trained him in symbolic interaction and field/qualitative research (Bryant \& Charmaz; Charmaz, 2000; Glaser, 1998; Nathaniel, 2009). The men forged a working relationship in San Francisco when Strauss hired 
Glaser to work on the dying study (Glaser, 1998). Each one’s unique educational background melded to allow the discovery of classic grounded theory.

Philosophical roots. Exploration of the philosophical roots of grounded theory has been the topic of many papers. In their writings, Glaser and Strauss freely acknowledge the influence of various teachers on their work, but never discuss the philosophical roots of grounded theory (Glaser, 2006; Nathaniel, 2009). Some write of the diverse learning experiences of Glaser and Strauss and the influence of those experiences upon the emergence of grounded theory (Bryant \& Charmaz, 2007; Charmaz, 2000). Many credit Blumer and symbolic interactionism as the philosophical roots of grounded theory (Annells, 1996; Annells 1997; Benoliel, 1996; Charmaz; Cutcliffe, 2000; Heath \& Cowley, 2004; Jeon, 2004; Marcellus, 2005; McCann \& Clark, 2002) Even Glaser (1998) notes that through Strauss he learned "the social construction of realities by symbolic interaction making meaning through self-indications to self and others”(p. 32).

Symbolic interactionism is "both a theory about human behavior and an approach to inquiring about human conduct and group behavior” (Annells, 1996, p. 380). Herbert Blumer receives credit for development of symbolic interactionism. However, Blumer's work is an extension and refinement of his University of Chicago teacher George Herbert Mead’s work. Mead's basic definition of symbolic interaction states "persons define themselves through social roles, expectations, and perspectives put on them by society and those within society” (Mead as cited in Annells, p. 381). In the development of symbolic interactionism, Blumer (1969) elucidated three basic premises, which follow.

1. Human beings act toward everything in their world (persons, categories of persons, institutions, physical objects, situations, guiding ideas) on the basis of the meanings that the things have for them. 
2. The meanings of things arise from social interaction with others

3. Persons use an interpretive process to handle or modify meanings as things are encountered. (p. 2)

The influence of symbolic interactionism is evident in classic grounded theory but so too are Glaser's mentors’ schools of thought and inquiry. It is in the constant comparative method where Glaser's educational background in qualitative math, theory development and explication de text becomes evident. Therefore, symbolic interactionism is an incomplete answer to the question of the philosophical roots of grounded theory. There is another option, Charles Sanders Peirce, a noted and prolific American philosopher. Nathaniel (2009) suggests Peirce is the philosophical root of grounded theory given that his philosophy and writings influenced Glaser and Strauss’s mentors directly and/or indirectly. Peirce’s philosophy of pragmatism or pragmaticism and writings are consistent with the original description of grounded theory by Glaser and Strauss and subsequent writing by Glaser (Nathaniel, 2009). Therefore, it is possible that Peirce provided the philosophical foundation for the discovery of grounded theory.

\section{The Method}

Classic grounded theory as a method has a number of simultaneously occurring steps or revolving steps as Glaser (1978) calls them. The researcher performs all of the steps but not sequentially as there is much going back and forth among the steps. The steps include data collection, coding, theoretical sampling, memoing, sorting, and writing. Further discussion of the steps follows. All the information regarding the steps originates from Glaser's 1978 Theoretical Sensitivity unless otherwise noted. 


\section{Data Collection}

Data collection begins when the researcher enters the field to interview participants. Glaser (1978) suggests that one enter fieldwork with an open mind and let the participants’ concerns emerge to become the research problem. The researcher enters the field without a formal interview schedule but rather a general "spill” question. Obviously, this question is relevant to the researcher's general interest but is not so narrow as to limit the participant's response. The participant shares what is of importance to him or her. The researcher asks a few follow-up questions in order to gain more in depth information or for clarification.

Most qualitative researchers tape interviews. Glaser (1998) strongly discourages the use of taping as it causes delay in coding secondary to the need to transcribe the tape. Taping also provides superfluous data. The goal is not full descriptive coverage but rather a conceptual theory (Glaser, 1998). Instead of taping, grounded theory utilizes field notes that the researcher writes immediately after leaving the field. Field notes contain observations of the participant as well as a summarization of what the participant said. Coding of the field notes follows.

Interviews with participants may not be the only source of data in a grounded theory study. The researcher has the option to use literature as a form of data as well. This literature serves to provide a source of comparison to the developing theory. Glaser (1978) advises against going to other sources of data until one has a stable framework for the theory. The decision to go other sources of data is a theoretical sampling issue. Data collection continues until the researcher attains saturation. Saturation occurs when no new properties emerge and the same properties emerge as the researcher codes and analyzes the data (Glaser, 1978). 


\section{Analyzing the Data}

Coding. Coding allows the conceptualization of latent patterns in the data. It starts once the researcher writes field notes from data collection and continues throughout the study. Fracturing of the data during coding allows the researcher to function at a theoretical level versus an empirical one. Coding is an important step in generation of the theory. Substantive and theoretical are the two categories of coding.

Substantive coding. Substantive coding conceptualizes the empirical data (Glaser, 1978, p55). Glaser describes two types of substantive coding: open coding and selective coding. Open coding occurs as the researcher codes every line of the field notes. This process provides for many codes. Once the researcher sees the emergence of core categories, it is time to switch to selective coding. Selective coding is the process by which the researcher only codes for the core variable.

Theoretical coding. Theoretical coding conceptualizes the possible theoretical substantive codes for fit into the theory (Glaser, 1978, p. 55). Theoretical Sensitivity includes several coding families. Glaser (1978) states that theoretical codes "weave the fractured story back together again” (p. 72). However, the story is now at a more conceptual or theoretical level.

\section{Theoretical Sampling}

Glaser and Strauss (as cited by Glaser, 1978) state that "theoretical sampling is the process of data collection for generating theory whereby the analyst jointly collects, codes, and analyzes data and decides what data to collect next and where to find them, in order to develop the theory as it emerges.” (p. 36). After some initial coding, the researcher returns to the field for more data collection in order to attain code saturation and integration into an emerging theory. 
This process occurs many times throughout the conduct of a study. The emerging theory guides the sampling and thus is unknown to the researcher at the start of a study. This additional sampling provides the researcher with increased theoretical sensitivity about the data, which ultimately aids in theory development.

\section{Memoing}

Memoing is the process in which the researcher immediately writes down conceptual ideas that come into mind. Glaser (1998) defines memoing as the "theorizing write-up of ideas about substantive codes and their theoretically related relationships as they emerge during coding, collecting and analyzing data and during memoing” (p. 177). Through memoing, field notes transform from descriptions to theoretical accounts (Montgomery \& Bailey, 2007). Memos initially occur as a byproduct of the constant comparative process; but later memos arise from every aspect of the research endeavor: readings, sorting, writing, and other memos.

Memo writing starts with initial coding and continues throughout. One stops whatever one is doing to memo when the muse strikes. It is in memoing that the data and coding rise to a more conceptual/theoretical level. There are four main goals of memoing according to Glaser (1978) in Theoretical Sensitivity: "the theoretical development of ideas, with complete freedom into a memo fund that is highly sortable” (p. 83). Glaser (1998) later wondered if those four goals as well as the twelve rules of memoing were too prescriptive and formal. Nevertheless, the goals are helpful and discussion of them follows.

Ideas. Memoing provides the researcher a means to get down on paper thoughts about the interconnections among the codes. Writing down ideas assists in the development of theory by a) raising the data to a conceptual level, b) allowing the start of operationalization of categories, c) generating hypotheses about the connections between categories, d) integrating the 
connections with other categories and, e) locating the emerging theory with other theories (Glaser, 1978, p. 84). Memoing takes the categories analyzed from the data and raises the abstraction for theory development.

Freedom. The goal of memo writing is not elegant prose but rather to get ideas out of the brain and onto paper. Great length is not a requirement. Glaser (1978) suggests that concerns about the mechanics of writing interfere with creativity and may lead to premature closure of analysis. Thus, freedom allows the researcher to be open to new thoughts and changes to ideas already written in a memo.

Memo fund. The memo fund is from where the researcher obtains all material for writing the theory. The size of the fund correlates to the size of the study. Glaser (1978) suggests that books, lectures and articles as well as a substantive theory may arise from the memo fund.

Highly sortable. It is imperative that the researcher write and organize the memos for ease of sorting. Given this requirement, Glaser (1978) makes many suggestions regarding organization of the memos. Suggestions include a descriptive title, highlighting of categories within the memo and discussion of connections if memo includes more than one category. In order to ease sorting, there is also a suggestion to use note cards or utilization of a media that allow the researcher to physically cut and paste the memos to create new combinations.

Other outcomes. Memoing is the activity that lays the groundwork for theory development. However, there are other outcomes from that benefit the researcher and ultimately the developing theory. Glaser (1978) notes that memoing may defuse preconceived ideas and slow the researcher down to allow thorough reasoning. Theoretical sampling decisions may arise from memos. Memoing allows the researcher to be open to related areas for later study. It cannot be overstated the importance of memoing to a grounded theory study. 


\section{Sorting}

Sorting occurs when the researcher conceptually organizes all the memos in order to develop an integrated theory. The researcher literally takes each memo, carefully considers the theoretical fit and places it with other theoretically similar memos on a large table or work surface (Glaser, 1998). Glaser (1998) recognizes that it can be overwhelming to see the large pile of memos and know where to start. He suggests that the researcher just dive into the pile to start sorting. It is important not to do so with preconceived outlines or ideas.

There are 11 analytic rules in Theoretical Sensitivity to guide the researcher through the process of sorting. The rules provide discipline for the researcher. These rules are not exhaustive. Glaser (1978) suggests others may emerge during the process.

Sorting is not a one and done phenomenon. Resorting occurs when integration of concepts falls apart. Sorting inspires the writing of more memos for sorting. This step is essential. Skipping this step may lead to a "thin and less integrated theory" (Glaser, 1978, p. 116).

\section{Writing}

Writing the theory starts once sorting is under way. The researcher needs to "write about concepts not people” (Glaser, 1978, p. 134). Writing clarifies theoretical relationships.

However, it also may generate more memos or even a need to re-sort. Finally, Glaser (1978) suggests that the researcher leave the finished product for a few days only to return with a fresh viewpoint for further editing.

\section{Outcome}

The outcome of a study using grounded theory methodology is a grounded theory. The grounded theory develops around a core category, which provides explanation for variation in a 
pattern of behavior (Glaser, 1978). A core category arises from the constant comparison process during coding, analysis of the data and theoretical sampling. Any type of theoretical code is a potential core category. Many core categories, however, are basic social processes.

Basic social processes (BSPs) reflect and summarize the systematically uniform patterns of peoples’ social lives over time (Glaser (1978). Basic social psychological process (BSPP) and basic social structural process (BSSP) are the two types of BSPs. As the labels indicate, a BSPP describes social psychological processes of individuals or groups of people such as the relationship of parents to children. Conversely, a BSSP pertains to social structural processes of organizations such as hospitals, hospices or home. It is not unusual for the intersection of BSPPs and BSSPs to occur, such as when examining the parenting of a dying child who requires care at home, hospice or hospital.

\section{Rigor}

The rigor of qualitative research methods continues to be the subject of debate in scholarly circles. Credibility, transferability, dependability, confirmability, trustworthiness, auditability, fittingness are all commonly used parallels to the criteria of internal/external reliability and validity of quantitative research (Beck, 1993 as cited in Chiovitti \& Piran, 2003; Lincoln \& Guba, 1985; Polit \& Beck, 2004; Roberts \& Priest, 2006, Ryan-Nicholls \& Will, 2009). While Morse, Barrett, Mayan, Olson \& Spiers (2002) propose that qualitative researchers reclaim validity and reliability by use of strategies to ensure rigor, Rolfe (2006) asserts that qualitative researchers must resist that adoption. Further, Rolfe argues that the push for universal criteria of quality for qualitative research is a futile notion given the diversity of non-quantitative methods. Nevertheless, whatever one's view, rigor is an issue that requires attention. 
Classic grounded theory has rigor. The rigor arises from the systematic approach of constant comparative method. Constant comparison requires the researcher to use induction and deduction to go back and forth between the data and the emerging core categories and theory for the purposes of theory generation (Marcellus, 2005). It is that going back and forth that provides the rigor of grounded theory. Glaser (1978) finds paradox in that notion that "grounded theory is a highly structured, systematic and rigorous; yet it allows the researcher to be free of the structure and rigor of more forcing methodologies” (p. 13).

How does one know if the theory is "right”? The criteria by which to judge any substantive grounded theory are fit, work, relevance and modifiability. Glaser and Strauss wrote about the first three criteria in the book, Discovery of Grounded Theory (Glaser, 1978). Modifiability is a newer addition to grounded theory based on Glaser's experience of using grounded theory over time. Fit occurs if the categories of the theory fit the data. Work refers to the ability of the theory to explain, predict and interpret what is happening in the area of study. Relevance is the idea that the theory is conceptually about the core concerns of the participants or area of study. Finally, a theory is modifiable if new data provides the impetus to modify the theory. Grounded theories are not verifiable but are rather modifiable to reflect an ever-changing world (Glaser, 1978; Glaser, 1998).

\section{Participants}

\section{Selection}

Qualitative researchers rarely if ever use probability sampling. Probability sampling is antithetical to qualitative research since the concern is with measuring attributes and relationships within a specific population not generalizability to the general population (Polit \& Beck, 2004). Non-probability sampling methods predominate in qualitative research. Many 
methods of non-probability sampling exist. Purposive sampling is one such method. The researcher using purposive sampling chooses participants for the study because that person possesses a characteristic relevant to the researcher's interest. This investigator used purposive sampling for the first 11 participants and then utilized theoretical sampling to select the last participant.

For this study, this researcher interviewed participants who were either the biological mother or father of an infant who died of a life-limiting congenital anomaly within the first 15 months of life. The participants had to be over the age of 18 and understood/spoke English. The baby of the participants had to meet the following criteria: (a) lived at least 3 weeks and no more than 15 months, (b) had a prenatal or post-delivery diagnosis of a life-limiting congenital anomaly, and (c) died within the last 10 years. At the onset, it was unknown how many participants would allow for saturation. The investigator estimated that number to be around 20 . However, saturation occurred with 11 participants. The investigator interviewed a twelfth person, a woman who was currently pregnant with a healthy baby, for theoretical sampling purposes. That interview brought the total number of participants up to twelve.

\section{Recruitment}

Recruitment of participants was a most challenging undertaking. The investigator used many different methods to recruit participants. The investigator first asked personal and professional acquaintances of their personal knowledge of someone who fit the inclusion criteria. That person was then asked to provide the potential participant with the investigator's contact information. Three participants were obtained in this manner.

The investigator planned to use snowballing to recruit more participants. It is not uncommon for people undergoing a challenging situation to reach out to others experiencing a 
similar situation. Therefore, it was likely that the participants of this study knew someone else who experienced the loss of an infant to a life-limiting congenital anomaly. Only one participant referred the investigator to another participant.

The investigator initially proposed to recruit participants through the placement of a recruiting advertisement in the local papers within in a 2-hour radius from the researcher's home base. However this method was not used. Given the sensitive nature of the topic, the investigator decided that advertising in a newspaper was not appropriate.

Another proposed method of advertising for the study was the internet. The investigator left messages with the web master of web sites such as the March of Dimes and livingwithtrisomy13.org and got no response. Share Pregnancy and Infant Loss Support Group responded but wanted the investigator to make too many major changes to the study. The Trisomy 18 Foundation did not allow advertisement on their Facebook or web page. However, the manager of community affairs at the foundation contacted families who met the study's inclusion criteria and lived in West Virginia. The investigator was then given the contact information of those who assented to participate. This method generated two participants.

Email was another internet source used to recruit participants. The investigator directly emailed a local participant. This participant had written a book describing her experience of being the parent of a baby with a life-limiting congenital anomaly. In the back of the book was an email address which the investigator used.

Finally, five participants were garnered through a local hospice. A member of the investigator's dissertation committee served as the medical director for an area hospice that provides end-of-life care to pediatric patients. The hospice contacted families who met the inclusion criteria to determine willingness to participate in the study. Five families indicated an 
interest in participating in the study and gave permission for the investigator to contact them. Hospice then supplied the investigator with the family's contact information. The investigator called and sent the information letter to the families. Four of the five families responded for a total of five participants.

Upon initial contact, the potential participants received an information sheet that: (a) outlined the general purpose of the study, (b) their right to not participate and (c) their rights should they decide to participate and (d) contact information for the investigator and the dissertation chair. No consent forms were used as the West Virginia University Institutional Review Board (IRB) labeled the study exempt. A copy of the participant letter is in Appendix A. Characteristic of Participants

The investigator collected some basic demographic data on the participants and their deceased neonates/infants. An overwhelming majority of the participants were female $(n=11)$ with only one male participating. All were Caucasian. Ten of the participants were currently married. Only one participant was no longer married to the father of the baby that had died. The other marriages remained intact after the baby's death. The one couple who both participated in the study was never married and no longer in a romantic relationship.

Characteristics of the baby. Basic demographic information collected on the baby included the baby's diagnosis, age of diagnosis and age at death. The participants' babies suffered from a wide variety of life-limiting congenital conditions. Those disorders included the following: mitochondrial disorder $(n=1)$, spinal muscular atrophy $(n=1)$, and teratoma $(n=1)$. Three babies had some form of a trisomy disorder, Trisomy $13(\mathrm{n}=1)$, Trisomy $18(\mathrm{n}=1)$, and Trisomy $22(n=1)$. Congenital heart defects was another classification of conditions experienced by the participant's babies, hypoplastic left heart syndrome $(n=2)$ and truncus ateriosis $(n=1)$. 
One participant gave birth to two babies with hypoplastic left heart syndrome. Finally, a couple of babies had no specific diagnosis other than multiple congenital anomalies $(n=2)$.

Most diagnosis of these life-limiting congenital conditions occurred prenatally $(n=4)$, right at delivery $(n=4)$, or in the first day or two of life $(n=1)$. The babies who lived the longest were also tentatively or definitively diagnosed later at five months of age $(n=2)$. The participant of the baby with a tentative diagnosis at five months of age did not get confirmation of the baby’s life-limiting disorder until the day of the baby’s death at nine months of age.

The life span of the deceased babies ranged from three days to 9 months of age. Over half of the babies died within the first two weeks of life $(n=6)$. The other babies died between three months to nine months of age $(n=5)$.

There did not seem to be any connection between the timing of the diagnosis and lifespan except for those diagnosed prenatally. Three out of four of the babies with a prenatal diagnosis of a life-limiting congenital condition died within the first four days of life. It is possible that this number was a reflection on the time the prenatal diagnosis allowed the parents to make decisions regarding resuscitation efforts at birth. The fourth baby with a prenatal diagnosis had a heart defect underwent surgery to ameliorate the problem but died at three months of age despite efforts to keep the baby alive.

The death of the baby occurred equally at two sites: in the home $(n=5)$ and the hospital $(n=5)$. One baby died at an in-patient hospice $(n=1)$. The hospital deaths occurred in two different types of units, the ICU $(n=3)$ and the mother's postpartum room $(n=2)$. However, one baby who died in the mother's postpartum room went to that room after ventilator withdrawal and lived less than an hour after arrival. 


\section{Procedures}

The investigator used grounded theory to investigate the parents' experience of parenting an infant who died of a life-limiting congenital anomaly. As previously discussed, grounded theory, as a method, consists of non-sequential steps. The following discussion presents data collection and analysis sequentially even though this did not occur because of the back and forth nature of grounded theory.

\section{Data Collection}

Data collection proceeded once a person agreed to participate. The interview was unstructured and opened with a broad general or "spill” question to allow the participant to share what was relevant relative to the general topic. The initial proposed spill question was "Tell me about being the baby's name mom or dad”. However, after a couple interviews, the initial question was replaced by "Please tell me your story". Then the initial spill question and additional follow up questions such as “Tell me more about .....” elicited clarification and/or elaboration. The investigator also asked a few demographic questions.

Conduct of the interviews occurred both in-person $(n=9)$ and via telephone $(n=3)$. The site of the in-person interviews was ultimately the decision of the participant. The most common location for the in-person interviews was the participant's home $(n=5)$. Participants wanted to show the investigator pictures and personal items of the child and so wanted to meet in their home. This particular environment provided rich data in addition to the spoken word. Other sites for in-person interviews included a private office at the participant's place of work $(n=2)$, the investigator's office $(n=1)$ and a fast food restaurant $(n=1)$. Telephone interviews took place at a time agreed upon by both parties. 
As previously noted, Glaser strongly discourages taping of interviews. This investigator previously conducted a pilot study of this study in which taping of an interview occurred. Field notes were first created without listening to the tape and then again after listening. Upon comparison, the field notes were very similar. As a result, the investigator received permission from the dissertation committee to not tape interviews in this study. However, the investigator wrote brief notes during the interviews to ensure the accurate recording of facts.

The writing of field notes immediately followed the interview. The investigator left the interview, found a private spot, and wrote field notes on a password protected laptop computer. Field notes included what the participant said as well as observations of non-verbal behavior and the environment as applicable. Analysis of the data followed. However, given the cyclical nature of grounded theory, data collection continued until saturation.

\section{Analysis}

Analysis of data began right after completion of the first field note. This analysis occurred simultaneously and continuously throughout the study. During initial open coding the investigator coded each line of the field notes. Concepts and categories emerged through the process of constant comparison. Memoing allowed the investigator to put to paper ideas regarding theoretical sampling and the relationships among the concepts and categories. The core category of living within the paradox emerged. Living within the paradoxes became the framework of the grounded theory, guided theoretical sampling, and spurred the generation of more memos. Sorting commenced once data collection finished and saturation occurred. Writing the theory followed sorting and spurred more memos and additional sorting. The outcome of this process was the grounded theory of Parents Living within the Paradoxes of Infant Death from Life-Limiting Anomaly. 


\section{Data Trail}

A data trail is an additional method to ensure rigor. In fact, it is a method suggested by some writers on the topic of rigor in qualitative research (Morse et al, 2002; Rolfe, 2006). The investigator labeled each field note and memo with a unique identifier. The identifiers were the date of the field note or memo followed by an F or M respectively. When the writing of more than one memo in a day occurred, the investigator added a lower case “a” as an additional identifier for the first memo. Subsequent memos identifiers on the same day progressed through the alphabet. Therefore, a third memo written on October 10, 2009 received an identifier of 101009Mc. Glaser does not specifically suggest the use of a data trail but does not condemn its use (A. Nathaniel, personal communication, June 11, 2009).

\section{Timeline}

The investigator obtained approval for the study from the investigator's dissertation committee in November of 2009. The Institutional Review Board (IRB) committee of West Virginia University labeled the proposed study as exempt in March of 2010. The Marshall University IRB concurred the same month. Data collection started upon approval by the IRB and continued until May of 2012 when saturation occurred.

\section{Protection of Participants}

The protection of participants is a vital concern. Upon approval by the dissertation committee, the investigator submitted the proposed study to the Institutional Review Board for Protection of Human Subjects of West Virginia University (see appendix B). Submission to the Marshall University IRB also occurred since the investigator is an employee of Marshall University. 
The investigator took a number of steps to protect participants in this study. First, potential participants received a letter informing them of their right to refuse to participate in all or any part of the study. The letter also outlined methods to protect privacy, such as the separation of identifying information from data. Also included in the letter was a statement that any publications or public presentations of findings of the study will not include their name without permission. During conduct of the study, all information was stored on a passwordprotected personal computer. The investigator kept any identifying information separate from the data.

The topic of the study was of a sensitive nature. There was concern that participants could experience distress recalling the life and death of their baby. The investigator planned to provide the participant with referral to a support group or a counselor. Payment of any counselor would have been the responsibility of the participant. None of the participants requested information about a support group or counselor from the investigator. Some were already seeing a counselor.

\section{Summary}

There is scant research exploring palliative/end-of-life care for dying infants and their families in order to address and improve care at the end-of-life. This current study used classic grounded theory as developed by Glaser and Straus. Use of a classic grounded theory was appropriate since little information existed about this phenomenon. This investigator followed the iterative steps of data collection, coding, theoretical sampling, memoing, sorting, and writing (Glaser, 1978). Throughout conduct of the study, the participants' rights were protected. The outcome of the study was a substantive theory: Parents Living within the Paradoxes of Infant Death from Life-Limiting Anomaly. 


\section{CHAPTER FOUR: RESULTS}

In this chapter, this author presents a grounded theory of Parents Living within the Paradoxes of Infant Death from Life-Limiting Anomaly (living within paradoxes). This grounded theory evolved from analysis of interview data collected from participants who had experienced the death of an infant from a life-limiting congenital anomaly. Analysis of the interview data used classic grounded theory methodology as described in Chapter 3.

\section{Summary of Living within Paradoxes}

In the first stage of living within paradoxes, the parent-to-be/parent is living in innocence. Living in innocence abruptly ends with the cutting point of getting the bad news. Getting the bad news occurs when the parent learns that there is a major health issue with the baby. This cutting point may happen prenatally or after birth.

The stage of being a good mom/dad in the new reality starts after getting the bad news. During the stage of being a good mom/dad in the new reality, the following processes occur: seeking answers, squeezing a lifetime into a moment, and letting go. These processes may be sequential but are typically iterative.

A second cutting point occurs at the death of the baby. Going on is the stage that follows the death of the baby. Saying goodbye, seeking meaning in the baby's life, keeping his/her memory alive, and living life are processes that take place during the going on stage. As with the second stage, the processes may be sequential or iterative. Figure 1 is a model of the stages and cutting points of the theory of living within paradoxes. 
Figure 1. Parents Living within the Paradoxes of Infant Death from Life-Limiting Anomaly consists of three stages with two cutting points.

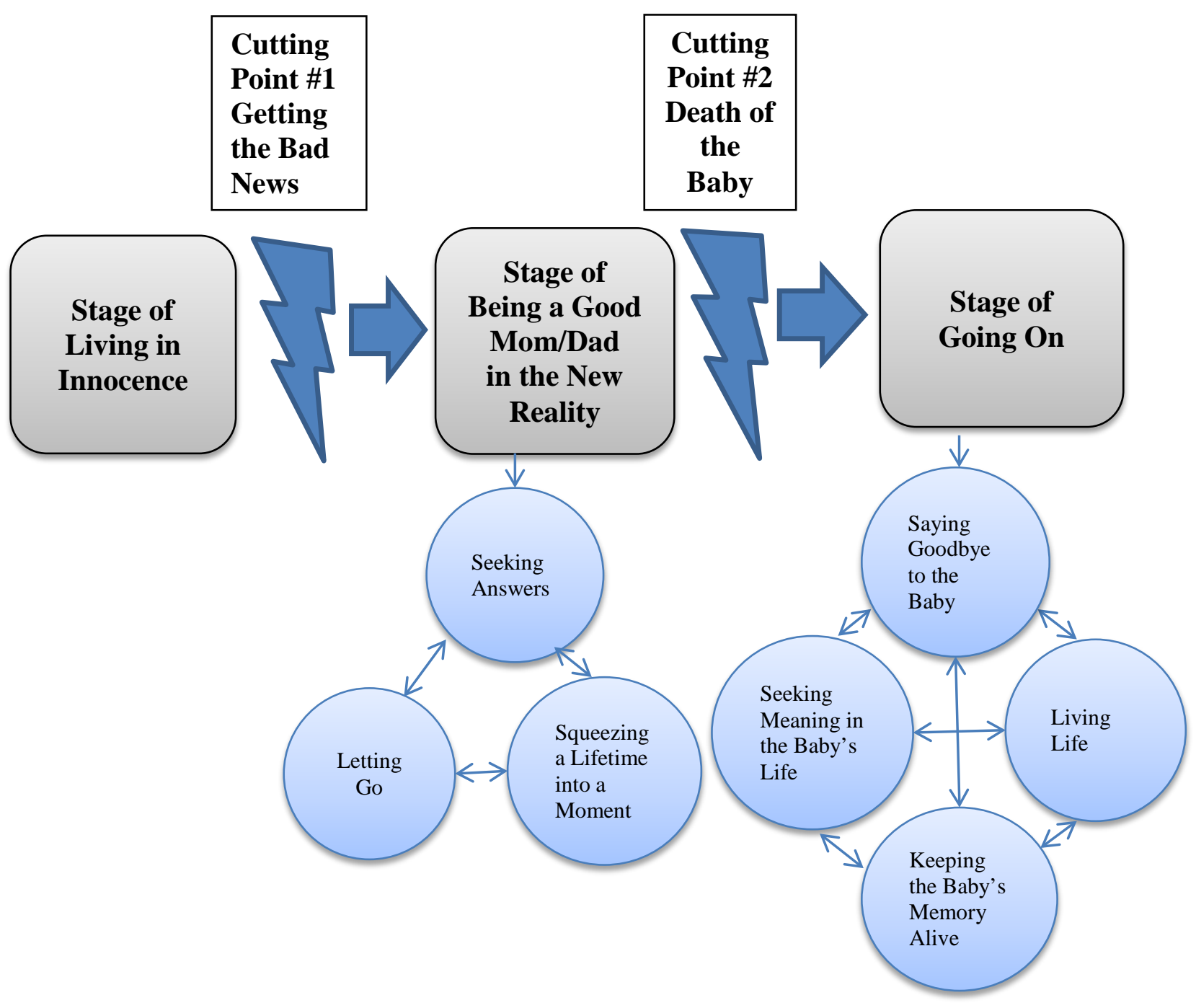

\section{Parents Living within the Paradoxes of Infant Death from Life-Limiting Anomaly}

Living within paradoxes is how parents go through the life crisis of giving birth to, parenting, and losing to death an infant with a life-limiting congenital anomaly. Paradox in this study is defined as "something (as a human being, phenomenon, state of affairs, or action) with seemingly contradictory qualities or phases” (Paradox, 1993, p.1636). Being the parent of a new 
baby who is going to die while still so new to life is the first paradox of the theory. From this first paradox others paradoxes arise.

The parent experiences many paradoxical situations and feelings throughout this time. Hope may be present in the midst of this seemingly hopeless situation. Being separated from the baby as well as being alone with the baby occurs. The parent may exhibit behaviors consistent with being a good mom or dad, but not feel like the baby’s mom or dad. Given an uncertain life expectancy, the parent tries to squeeze a lifetime of being with a baby into a short time. Memories evoke both happiness and sadness. Further discussion of these and other paradoxes takes places during discussion of the stages and processes of the theory.

While sharing their stories, the participants repeatedly shared about paradoxical situations and emotions related to being the parent of an infant with a life-limiting congenital anomaly. One participant summed up the paradox of hope in the midst of a hopeless situation by saying "Every night we thought she was going to die, every morning we thought she was going to live”. Paradox was evident when participants had a smile on their face and tears in their eyes when sharing a specific memory about the baby. Other specific instances of paradox shared by the participants follow later in the discussion of the theory.

\section{Stage of Living in Innocence}

Living in innocence is the first stage of the theory of living within the paradoxes. During this stage the pregnant woman or father-to-be awaits the birth of a healthy baby or the new parent enjoys a seemingly healthy baby. The physical discomforts related to pregnancy or childbirth are minimized or tolerated because the outcome, a healthy baby, is worth it. The parent feels optimistic and plans for a future that includes the new baby. These plans include opportunities, such as travel and schooling, that the parent wants to provide to enrich the baby's 
life, even though those are years away. The parent may fantasize about the kind of person the baby will grow up to be and the wonderful and constructive things he/she will do in life. It is a time full of hopes and dreams. However, this stage ends abruptly with the diagnosis of a lifelimiting congenital anomaly or getting the bad news. That diagnosis may occur during pregnancy or after delivery of the baby.

Most of the participants described the pregnancy as normal or the baby as healthy. The pregnancy/new baby was greatly desired by most participants. A couple participants discussed using some form of reproductive technology to conceive. For a couple participants, the pregnancy was not planned. Planned or not, the participants assumed that the outcome of the pregnancy would be a healthy baby. The participants talked briefly about this time, focusing instead on the rest of the story.

\section{Cutting Point One: Getting the Bad News}

The stage of living in innocence ends abruptly with getting the bad news. Getting the bad news occurs when the parent learns that there is a major health issue with the baby. This cutting point may happen during pregnancy or after the birth of the baby.

Getting the bad news is a sentinel experience. It is the moment when the ideal of the perfect baby leaves and forces a new reality on the participants. Anticipation becomes anxiety, fear, guilt and dread. Joy is replaced by sadness and grief. Dreams and plans are forever altered.

All participants talked about getting the bad news. A few participants discussed sensing something was wrong prior to getting the bad news. However, this did not change the devastation felt when getting the bad news. 


\section{Stage of Being a Good Mom/Dad in the New Reality}

The stage of being a good mom/dad in the new reality starts with getting the bad news. Living in innocence is forever gone. The parent is now left with the new and awful reality that the baby will or is likely to die. It is not something the parent can avoid. The new reality must be faced. The parent must deal with the demands that are present for all new parents of a baby. However, there are additional and unique challenges that follow the diagnosis of a life-limiting congenital anomaly in the baby. This is the stage of being a good mom/dad in the new reality. The stage may last for minutes, hours, days or months. Being a good mom/dad in the new reality always ends with the second cutting point: the death of the baby.

Three major processes occur within the stage of being a good mom/dad in the new reality. Seeking answers, squeezing a lifetime into a moment, and letting go are the three major processes. Other properties occur within the three major processes. Discussion of the properties follows in subsequent sections. Figure 2 is a depiction of the stage.

A paradox of this stage is that some parents do not feel like a mom or dad despite demonstrating parenting behaviors during the processes within being a good mom/dad in the new reality. This seems especially true if the baby spends a majority of a brief life in the NICU. "I did not feel like her mom, I only got to change her diaper and give her one bath” stated one participant. A father stated "Sometimes I felt like her dad". 
Figure 2: Stage of Being a Good Mom/Dad in the New Reality: A stage with 3 processes

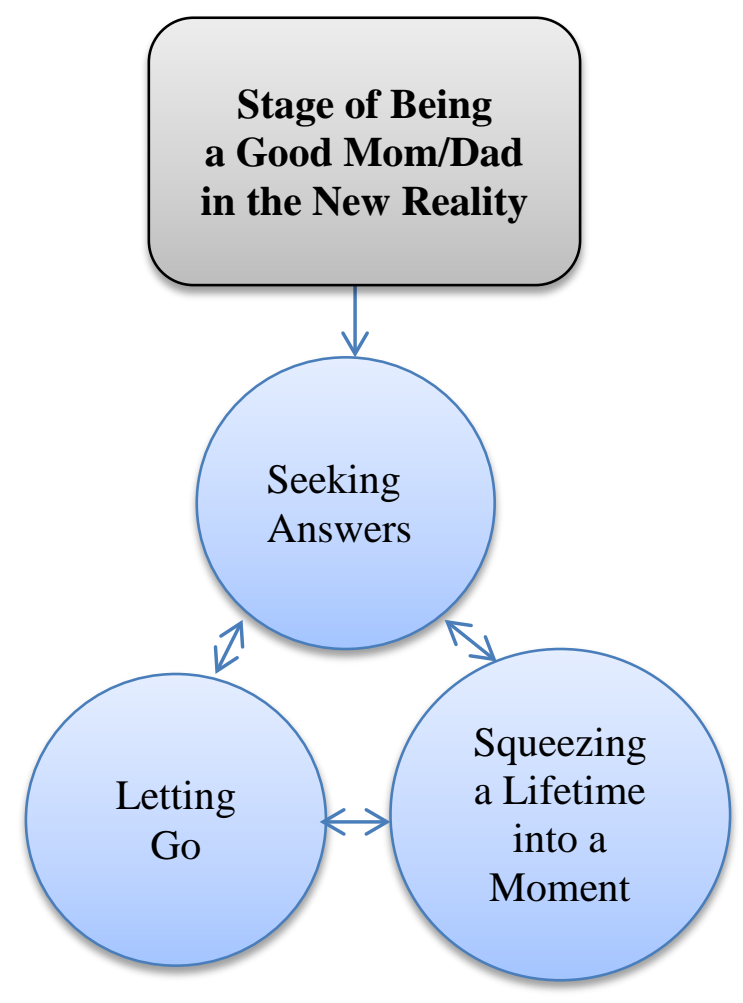

\section{Seeking Answers}

The parent, being a good mom/dad, starts the process of seeking answers to address the issues raised by getting the bad news. Therefore, seeking answers is typically the first process that occurs in the stage of being a good mom/dad in the new reality immediately after getting the bad news. However, the process of seeking answers is iterative and happens as long as the baby lives. During the process of seeking answers, three related properties occur: maintaining hope, being separated from the baby, and making decisions. Figure 3 depicts the process of seeking answers and the three properties of this process. 
Figure 3: Seeking Answers: A process with 3 properties

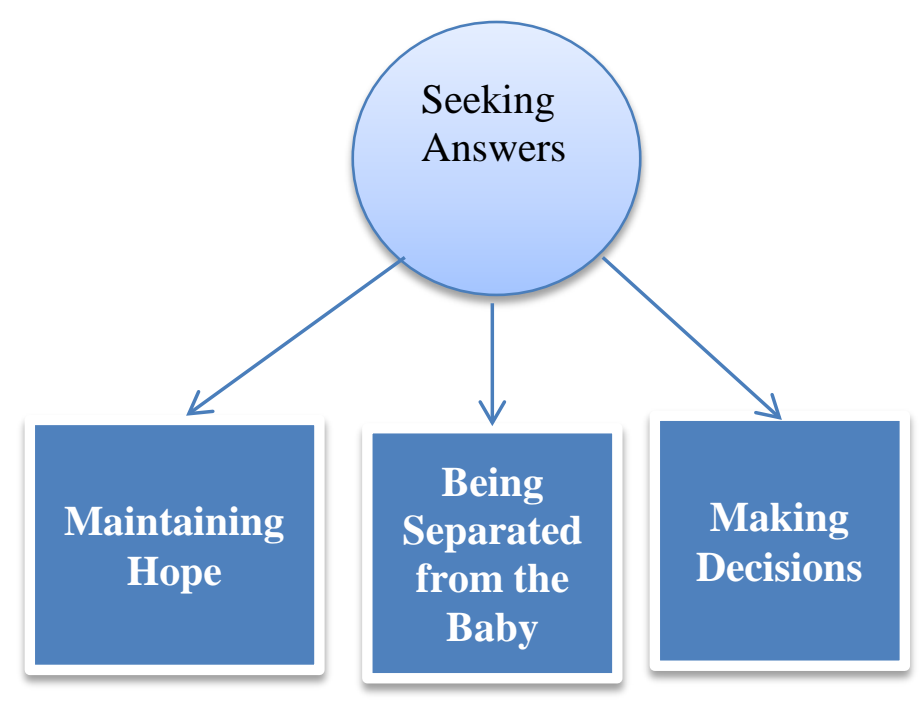

Seeking answers may mean trying to find out what is wrong and to save the baby.

Transfer and admission to specialized units is another means of seeking answers. How to explain the situation to the siblings of the baby and help those children cope is another aspect of this process. A final means of seeking answers is finding how to best care for a dying baby.

Referral to a high-risk perinatal or neonatal/pediatric specialist is an element of seeking answers. A referral to these specialists usually occurs when problems arise in the woman's pregnancy and/or the baby develops problems after birth. These specialists may be local or in locations far from home. The parent goes to the specialist seeking answers about what is wrong with the pregnancy and/or baby. Hope exists that the specialist can make everything all right. All participants received or sought referral to high-risk perinatal or neonatal/pediatric specialists. One participant described searching the internet for the best pediatric heart doctors and decided to go there for care even though it was eight hours from home. Another called a specialist 
several hundred miles away every day for three months trying to get an appointment even though the baby was receiving care from a group of specialists closer to home. One participant was still seeking answers far from home the week before her son’s death.

Transfer and admission to specialized units, high risk antepartum for the still pregnant woman and neonatal/pediatric intensive care units for the baby, may take place. Admission of the baby to the NICU/PICU is very common. These transfers and admission are another means of seeking answers. The parent or baby may spend a great deal of time in these units which also may be far from home. Some babies spend the majority or all of a brief life in the NICU. One participant spent five weeks in a high-risk antepartum unit three hours from home away from her husband and children. All of the babies, except one, spent a portion of their brief lives in an intensive care unit. One baby had at least seven surgeries and spent all five months of life in a NICU. Another baby, who only lived 3 days, spent all but the last hour of life in a NICU.

The parent may have concerns about the living children who are siblings of the baby. Seeking answers may include questions on how and what to tell the baby's sibling (s) about the baby's condition. There may concerns about the logistics of who will take care of the siblings if the parent has to go far away for care. The child(ren) may be very young with a limited ability to understand death because of the developmental stage. The parent may need assistance in trying to prepare the siblings for the baby's death. Many of the participants had other living children and wrestled with these issues. One participant shielded her very young children from the reality of the situation while another participant asked the local hospice to come to the siblings’ classrooms and discuss death. Yet another participant asked for the sibling's input about bringing the baby home to die. 
Seeking answers also means searching how to best care for a baby that is likely to die. This type of seeking answers occurs once the fetus/baby has a diagnosis of a life-limiting anomaly and the parent experiences letting go. Options for end-of-life care for the baby may include staying in the NICU/PICU, in-patient or home hospice care or rarely care on the regular mother-baby unit. Many of the participants received hospice care. One participant and her family sought out services from a perinatal hospice that provided care to the participant and family during the pregnancy and through the birth and death of the baby.

Maintaining hope. A property within seeking answers is maintaining hope. The parent hopes that somehow that something can be done and that the baby will live. Some parents never give up hoping for a miracle despite a grim prognosis. Others claim to know that the baby will not come home. However, hope is implicit in the lengths that a parent goes to save the baby. There is paradox inherent in maintaining hope in the midst of a seemingly hopeless situation.

Only two of the participants overtly stated never giving up hope. Spending five weeks in a high-risk antepartum unit away from a husband and children was just one example of the lengths gone to save a baby. Another participant took the baby to a large nationally recognized children's hospital five hours away from home just a week before the baby’s death. The baby was under the care of hospice at the time.

Being separated from the baby. Being separated from the baby often occurs as a consequence of seeking answers. Admission and transfer to specialized units means separation from the baby. Distances, unit policies, severity of the baby's illness and/or health complications of the mother are sources of the separation. No matter the cause, the separation is a source of stress. The paradox in this situation is being a good mom/dad in the new reality to save the baby leads to being separated from the baby. 
A baby born with a life-limiting congenital anomaly may require extensive resuscitation and immediate transport to the NICU right after birth. As a result, the parent may only get a quick glance and touch of the baby prior to transport. If the baby's condition is serious enough to warrant transfer to a tertiary center, the parent may be in a different hospital or even different city from the baby. Thus the parent experiences being separated from the baby. Participants talked about seeing the baby only briefly right after birth because the baby was quickly taken to the NICU for care. Some babies were transferred to an intensive care unit in another hospital. While some of these referral hospitals were in the same town, others were several hours away. One participant's baby was transferred twice because the baby was too sick for the first referral hospital.

Distance is not the only sources of separation for parents whose babies are in the NICU/PICU. Despite a philosophy of family-centered care and open visitation policies, most NICU/PICU's present barriers to a parent who want unlimited access to the baby. Often the baby's condition is so critical and tenuous that a simple touch by a parent is too much stimuli for the baby. One participant could not hold her baby because the baby’s hydrocephalus was so severe. To hold the baby would cut off the baby’s airway.

The condition of the mother is also a potential source of separation from the baby. A cesarean delivery or complications prior to, during or after delivery may require a hospital stay of a day or two. The complications and hospital stay keep the mother from being able to be at the bedside of baby transferred to another facility. One participant described not waking up for several hours after a cesarean delivery secondary to having general anesthesia. Yet another described almost dying during surgery due to blood loss which delayed being able to go to the NICU to see the baby. One physician made a participant promise to not go to the referring 
hospital two hours away for a couple of days after discharge. It was a promise that the participant honored.

Alternatively, once diagnosed with a life-limiting congenital anomaly, the parent may opt for the baby to receive care in an inpatient hospice. This too may be a source of separation from the baby since the location of inpatient hospices tend to be urban. One family experienced separation from the baby secondary to placement in an inpatient hospice almost two hours from home. The father and mother described going to visit the baby every few days. Job and home responsibilities and finances kept the family from being with the baby full time. This family was not with the baby when death occurred.

Making decisions. One result of the knowledge gained from seeking answers is the need for making decisions. Being a good mom/dad in the new reality requires that parents make many decisions over the course of the pregnancy and/or baby's short life. Some of these decisions are literally life and death ones.

Physicians often offer those that receive prenatal diagnosis of a life-limiting congenital anomaly the option of elective termination of the pregnancy. The parent must decide whether to terminate or continue the pregnancy with the knowledge that the baby will die anyway. One participant stated "He gave us options. Termination was one; I would not kill my baby. So we continued the pregnancy”. Another participant, who would not terminate, chose perinatal hospice instead.

Another decision that a parent with a prenatal diagnosis of a life-limiting anomaly may make is about resuscitation efforts at birth. The parent has to decide whether to allow or refuse heroic resuscitation of the baby at delivery. Resuscitation does not guarantee that the baby will live to adulthood, but buys time to allow confirmation of the life-limiting anomaly. Then the 
parent has knowledge with which to make further decisions regarding the baby's care. The paradox of this decision is that the parent in effect is thinking about death at the time of birth. Refusing resuscitation occurs after the parent experiences letting go. A couple participants chose to not allow resuscitation at delivery. For one participant, the decision not to resuscitate was part of a detailed birth plan about the baby’s care.

A parent with a prenatal diagnosis of life-limiting congenital anomaly may also opt to receive care from a perinatal hospice. Perinatal hospice is a newer model of care that provides support and care to a parent/family who do not want to abort a fetus diagnosed with a lifelimiting anomaly. However, that option is not universally available. One participant did opt for perinatal hospice, but had to travel eight hours from home to receive that care.

A parent who has a baby diagnosed with a life-limiting congenital anomaly after birth may decide at some point to either take the baby off the ventilator or have the physicians write a Do Not Resuscitate (DNR) order. This decision may take place right after diagnosis of the lifelimiting anomaly or may occur after a long struggle to save the baby. The parent is letting go. One participant upon getting the diagnosis said "I told them to take her off the vent, bathe her and bring her to me". Another reflected on the decision by saying "We decided to make her a DNR-it was not an easy decision but she was so sick”.

Some babies do not die within a few hours of being taken off the ventilator. That situation requires more decision making. The parent has to decide to stay at the hospital or seek hospice care. Hospice care is an option for some families but not all. However, to accept hospice care leads to another decision. The parent now has to decide whether to opt for hospice care at home or in an inpatient hospice. Most participants chose home hospice care while one family chose inpatient hospice. 
The decision whether to feed the baby is important to the parent. Parents insist on feeding the baby so the baby does not experience hunger or starve to death. This is true even if the baby is only likely to live for a day or two. Feeding is a life-sustaining behavior. So feeding a dying baby is paradoxical. Some babies eat well until right before death. However, the baby may be too sick or weak to breast feed or take a bottle. So feeding sometimes requires the use of an orogastric feeding tube, a syringe or finger. The parent has to decide about breast milk or formula. All but one participant discussed the issue of feeding the baby. A few pumped so that the baby could have breast milk.

\section{Squeezing a Lifetime into a Moment}

Squeezing a lifetime into a moment is a vital process that occurs during the stage of being a good mom/dad in the new reality. During this process the parent literally tries to squeeze a lifetime of being with the baby into whatever brief time the baby is alive. That time may be minutes, hours, or months (this study only included parents with babies that died between 48 hours and 15 months of life). Consequently, this process may occur over a very brief or a more extended period of time. No matter how long this period may be, it is never enough time. The parent wants more time with the baby.

Three main properties occur during squeezing a lifetime into a moment. Keeping vigil, creating memories, and seeking normalcy are those three properties. These properties do not occur in any particular order within squeezing a lifetime into a moment and often occur simultaneously. Two sub-properties occur during the property of seeking normalcy: being alone with the baby and taking the baby home to die. Figure 4 is a depiction of the process of squeezing a lifetime into a moment with the 3 properties and 2 sub-properties. 
Figure 4: Squeezing a Lifetime into a Moment: A process with 3 properties and 2 sub-properties

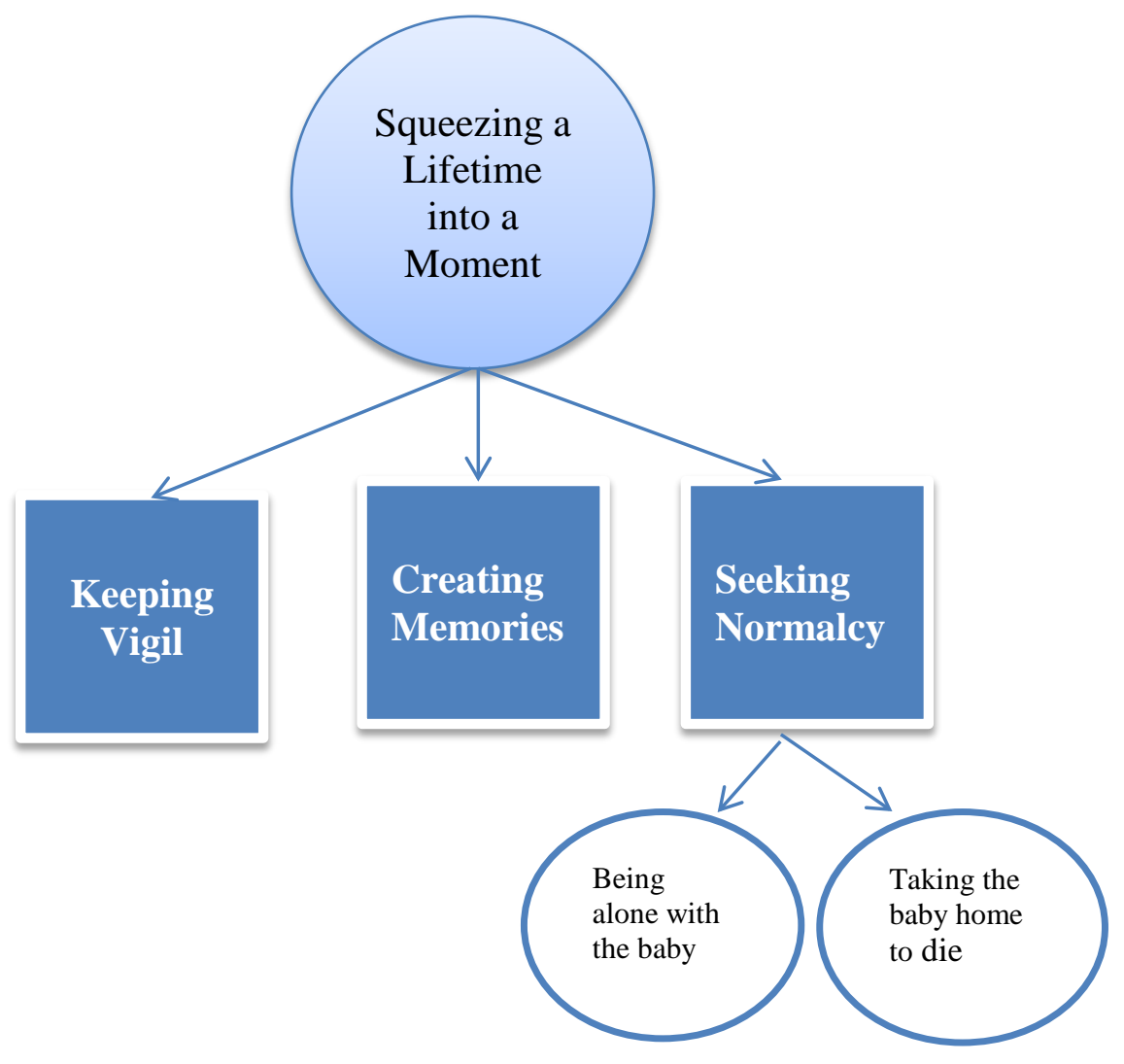

Squeezing a lifetime into a moment is another paradox in the theory of living within paradoxes. How does one squeeze a whole lifetime into a short period of time? Despite the obvious contradiction, the parent tries to do just that.

Keeping vigil. Central to the process of squeezing a lifetime into a moment in the stage of being a good mom/dad in the new reality is being with the baby. Keeping vigil is a hypervigilant state of being with the baby. The parent never allows the baby out of sight. If and when the parent sleeps, others receive the appointment to watch the baby. Other behaviors may include closely watching the baby breathe and holding the baby all the time. This differs from what an anxious new mom exhibits. The anxious mom is still living in innocence and assumes 
there will be a lifetime with the baby. Whereas the parent in the process of squeezing a lifetime into a moment knows there is a future without the baby.

The parent keeping vigil watches for any sign that there is improvement or worsening of the baby’s condition. Reinforcement of maintaining hope occurs if the baby demonstrates any improvement or remains stable. Conversely, as the parent sees the baby's condition worsening, the process of letting go begins. Any changes seen during keeping vigil may require the parent to revisit making decisions.

All participants talked about keeping vigil. "I did not sleep much while she was here. I watched her all the time" said one participant. Some participants talked about sitting at the baby's bedside in the NICU/PICU for hours, only leaving to get some sleep. Babies at home were not left alone and either slept with the parent or was held by someone as the parent slept.

Creating memories. Creating memories is another property in squeezing a lifetime into a moment in the stage of being a good mom/dad in the new reality. This property may occur anytime during this stage. Since the parent has an unknown and limited time with the baby there is an urgent need for creating memories that will last a lifetime. Once the baby is gone, the parent will only have the memories.

The parent creating memories does so by spending time with the baby. Others spending time with the baby may include the baby’s siblings, grandparents, other extended family and friends. During this time, the parent will hold, dress, feed, and/or provide other types of care for the baby. Siblings, others family member and friends may hold the baby as well, but not too long as the parent wants all the time possible with the baby. Family members, hospital staff, or a volunteer photographer from the "Now I Lay Me Down to Sleep” program can take pictures of 
the baby with the parent, family and friends. The parent may also opt to create a special memory by having the baby baptized or dedicated to God during this time.

Creating memories may involve creation of mementos, which become important in the process of keeping the baby's memory alive during the stage of going on. Pictures are one type of memento already mentioned. Other mementos created during creating memories may include making a casting of the baby's hands or feet or putting hand or footprints on a card or other item. Items used or worn by the baby may also become mementos later.

Creating memories was done by all participants. All discussed having siblings, family, and friends hold and spend time with the baby. Picture taking was also a method of creating memories done by all participants. Two participants made sure this author knew that the baby was baptized or dedicated to God. One participant's detailed birth plan made sure that the baby's older siblings were able to see the baby right after birth. All participants showed the author at least one memento of the baby.

There are a few paradoxes present in this process. One is that the memory of the baby will live longer than the baby was on earth. Another paradox is that the parent is trying to create positive memories of a time that is, at best, negative. Lastly, sharing the baby with others during creating memories is contradictory to the parent's desire of being alone with the baby.

Seeking normalcy. The third property within squeezing a lifetime into a moment is seeking normalcy. As with keeping vigil and creating memories, seeking normalcy may occur anytime in the process of squeezing a lifetime into a moment during the stage of being a good mom/dad in the new reality. Seeking normalcy is the attempt by the parent to find or create a sense of order in an out-of-control situation. In that attempt one finds yet another paradox. 
Seeking normalcy is, in part, the process whereby the parent overlooks obvious outward signs of the baby’s problems. The manifestations of seeking normalcy may include comments about how much some part of the baby, such as toes or eyes, resembles the other parent or someone else in the family. This focus on the normal occurs without immediate acknowledgment of any obvious physical manifestations of the life-limiting congenital anomaly. One participant discussed seeing the baby for the first time and thinking that the baby looked just like her husband without mentioning the absence of both ear lobes.

There is an element of denial of the situation in this aspect of seeking normalcy. Denial may give the parent time to process the reality of the situation. This seems especially true if getting the bad news occurs immediately after the birth of the baby.

Another aspect of seeking normalcy is when the parent desires the baby to exhibit a specific behavior usually seen in a healthy baby. The desired behavior may be for the baby to cry, to suck/nurse, or to open his/her eyes. This search for normalcy may partly explain what motivates a parent's decision to want the baby be fed. Whatever the behavior, the parent searches for a sign that the baby is normal. A couple of participants talked about being bothered that the baby never cried. Others talked about hearing the baby cry only once. The husband of another participant begged the baby to open her eyes just once. This participant was convinced that the baby saw her husband when the baby opened one eye right after the request.

Seeking normalcy also occurs as the parent performs basic baby care tasks such as changing a diaper or feeding the baby. These seemingly mundane tasks take on a new significance when the baby is sick in the NICU and/or not expected to live. Often the performance of these little tasks is what the parent remembers after the baby is gone. For some 
parents, doing these tasks are indicators of being a good mom or dad. All participants talked about some aspect of performing basic baby care.

Finally, seeking normalcy may include the being in or creating an environment that is more home-like than the highly technical intensive care unit. The parent wants the time spent with the baby to be of good quality. To have this quality time, the parent may demand being alone with the baby or opt for taking the baby home to die.

Being alone with the baby. During the property of seeking answers the parent may experience being separated from the baby. As discussed previously, the baby's condition may require transfer or admission to a NICU/PICU which restricts the parent's access to the baby. In response to this separation during the property of seeking normalcy, the parent claims being alone with the baby. Being along with the baby and being separated from the baby are two paradoxical properties within the theory.

It is a challenge, even a contradiction of terms, for the parent to experience being alone with the baby in the intensive care unit. There are always all types of health care personnel around. Sometimes the structure of the NICU impedes the ability of a parent to be alone with the baby. Many older NICU's are still one large single room with little privacy. Parents who have a dying baby may be relegated to a small private area away from the main NICU or may just have rolling curtains placed around the baby's area. Many newer NICUs are set up with private rooms that make being alone with the baby more likely.

Being alone with the baby may occur after letting go takes place and heroic measures are never initiated or stop. The baby may or may not be actively dying. Being alone with the baby is in some aspects similar to the processes of keeping vigil and creating memories within squeezing a lifetime into a moment. 
Finally, being alone with the baby may only occur once the baby is actively dying and/or after taking the baby home to die. Even at home the parent may need to claim alone time with the baby. Well-meaning family and friends there to support the parent may impede on the parent's alone time with the baby.

Many participants talked about being alone with the baby as something special, especially those in the hospital in NICUs. There was never time alone because of all the health care personnel around. Time alone was cherished. One participant stated, "The day she died, they took her off the vent and I sat with her all day. I did not want anyone else there. I knew she was not doing well, I just wanted her to myself”. For those at home it was important to claim alone time with the baby apart from the family. "We passed him around to the family there for them to say goodbye to him. Then we took him to our room to have private time. We laid him on our bed between us” related one participant.

Taking the baby home to die. A property that may occur as an element of seeking normalcy is taking the baby home to die. Taking the baby home to die does not occur with all families, but is a significant event for those that do. The event is full of uncertainty and often scary for the parent. Taking the baby home to die seems to represent the parent taking ownership of the baby. At home, the parent does not have to share the baby with the nursing and medical staff.

Taking the baby home to die may present challenges because of the care and observation the baby's condition requires. Paradoxically, in some ways the baby requires more care than a healthy baby, in other ways less. The parent needs support with these care needs, no matter how simple. Home health or hospice may provide this support. 
Babies with some life-limiting congenital anomalies have variable lifespans. These babies may not be actively dying at discharge from the hospital and may live for a few hours, days, or months. The severity of symptoms and care required also may vary among these infants. Some babies require tube feedings every three to four hours, while others may need oxygen, suctioning, and medication administration in addition to feeding. Some parents feel capable of providing this care whereas others feel overwhelmed. One baby with a neuromuscular disorder required oxygen and a feeding tube. That participant shared that it was easy to take care of the baby at home and had the help of hospice. Another participant felt uncomfortable about the baby's many care needs, particularly care of the tracheostomy. That baby ended up in back in the hospital and ultimately in an inpatient hospice.

The other situation of taking the baby home to die occurs when the baby lingers when taken off the ventilator. The parent is given the option to take the actively dying baby home or stay in the hospital. Some parents want to bring the baby home to die; others chose to stay in the hospital. One participant decided to take the baby home to die after a nurse in the NICU said going home was an option. Another participant sought the permission of the baby's siblings prior to bringing the baby home to die. None of the participants who brought the baby home to die voiced regret over the decision.

The process of letting go often precedes the parent taking the baby home to die. However, as discussed later in this chapter, letting go may not be a one-time process. So letting go may occur after the parent takes the baby home. As the parent takes the baby home to die, squeezing a lifetime into a moment becomes primary. The paradox of this situation is that this property occurs as a part of seeking normalcy. There is nothing normal about taking the baby home to 
die. Most families who bring a baby home from the hospital expect the baby to thrive and grow. These parents know that the baby is going to die.

\section{Letting Go}

Letting go occurs when the parent allows for withdrawal of heroic measures and the initiation of palliative or hospice care. The process of letting go also occurs when a parent decides during the prenatal period to forgo the initiation of resuscitation measures at birth. So letting go may occur prior to birth or any time after the birth of the baby. The parent understands that it is futile to initiate or continue heroic medical intervention to save the baby's life. The end result is the same, the baby dies. Instead of cure, the focus of care becomes the prevention or ease of suffering. Quality of life versus quantity of life becomes primary.

Two participants let go prior to delivery. One sought hospice care for the baby and took the baby home to die. The other obtained the services of a perinatal hospice; that baby died in the hospital during the process of discharge to home. Letting go occurred post-delivery for the others. Some babies went home to die while others died in the hospital. A couple participants shared telling the baby that it was ok to go.

Letting go may not occur just once. Rather the parent may vacillate between the paradoxical processes of maintaining hope and letting go. This vacillation may occur if the baby experiences a good hour, day, or week. The parent who vacillates looks for any little sign that the doctors are wrong. The less symptomatic and longer the baby lives, the more likely that this vacillation will occur. One participant epitomized this paradox when sharing the following:

He had the best week, a wonderful week. I began to wonder if I should give up on him since he was fighting so hard to stay. So we packed up and drove to the Cleveland Clinic. After being admitted, they sent the 
palliative care team in to see us instead of the specialist. We decided to go home.

This baby was already under hospice care at home and died within the week.

During the process of letting go the parent shifts into the process $f$ seeking answers and property of making decisions. The parent seeks answers and makes decisions about how and where to best provide care for the baby who is actively dying or will die sometime in the future. Additionally, the process of squeezing a lifetime into a moment takes on a sense of urgency as the parent realizes that time left with the baby is very short.

\section{Cutting Point Two: The Death of the Baby}

The death of the baby is the second cutting point in the theory of living in paradoxes. The baby takes a final breath and a heart stops beating. Death occurs. The stage of squeezing a lifetime into a moment ends.

Death is at times a paradoxical moment. The parent wants the baby to stay alive but also wants the suffering to stop. So when death occurs, the parent experiences feelings of grief and sadness. However, the parent may also feel relief that the baby's suffering is over.

All participants, except one couple, discussed being with the baby at the moment of death. When discussing the moment of death, all cried or became tearful. One participant shared that she wanted to scream at the moment of the baby's death but could not. "I was so happy that her battle was over. I knew she could breathe” was the statement of another participant. Yet, tears welled up in this participant eyes when sharing about the moment of the baby's death. 


\section{The Stage of Going On}

The stage of going on begins with the death of the baby. Going on does not mean getting over the loss of the baby. Rather, going on involves doing what needs to be done to live life. The parent goes back to work, a marriage, and parenting (if applicable). However, life is forever changed.

The first process that happens in the stage of going on is saying goodbye to the baby. Seeking meaning in the baby's life, keeping mementos, and living life are processes that occur iteratively after saying goodbye. All four processes occur throughout the stage of going on. Please see figure 5 for the depiction of the stage of going on.

Figure 5: Going On: A stage with 4 processes.

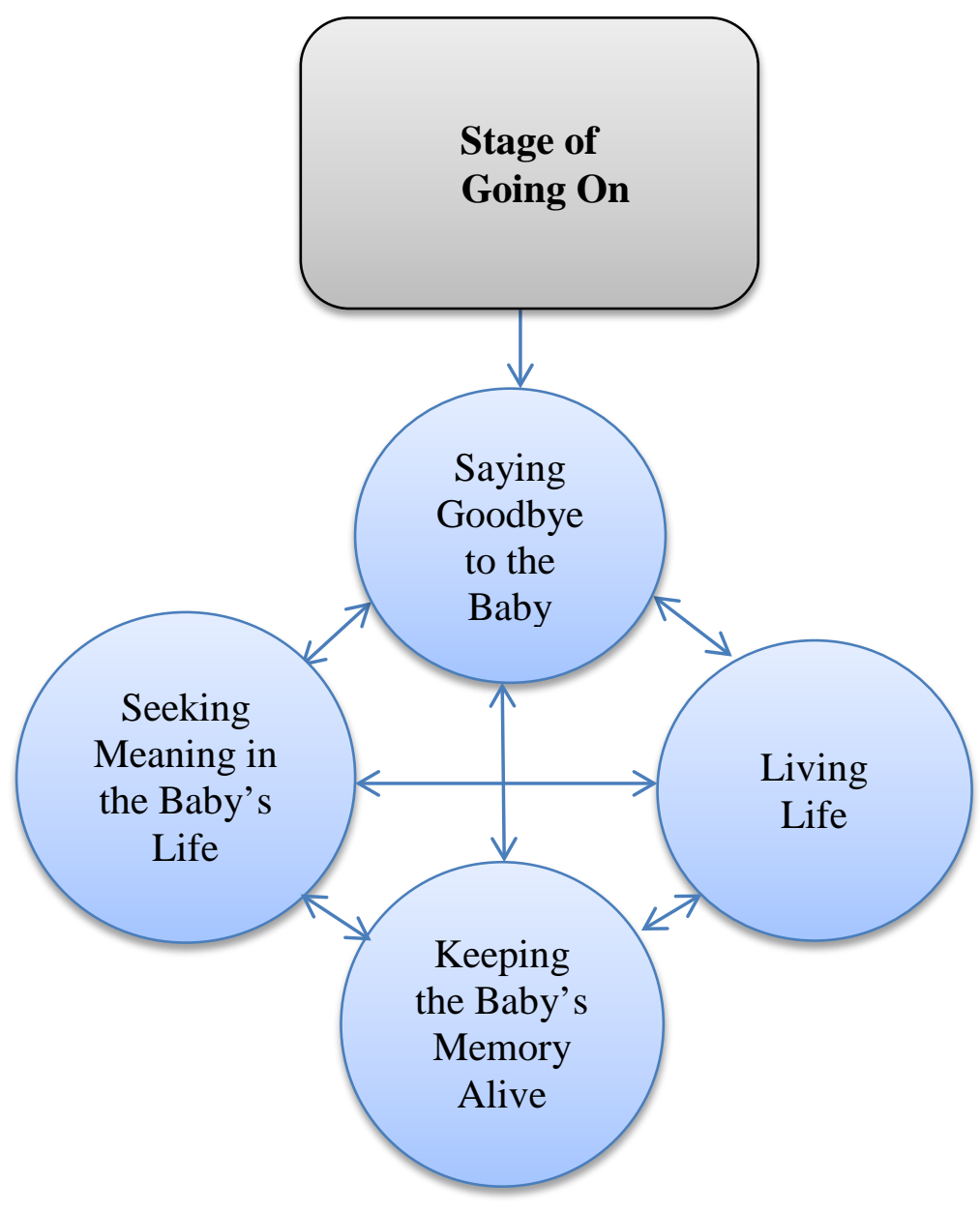




\section{Saying Goodbye}

Saying goodbye is the first process that occurs after the death of the baby. This process also continues throughout the stage of going on. Parents react to the death of the baby and feel the loss. During this time the parents prepare for a physical separation from the baby.

A first separation occurs when the baby goes to the morgue or to the funeral home.

Leaving the hospital without the baby is a difficult experience for the parent. It is not natural to deliver a baby and go home with empty arms. For those parents who care for the dying baby at home, it is hard to surrender the baby to another. Prior to this first separation, the parent may bathe, dress or hold the baby.

Participants whose baby died in the hospital talked about how hard it was to leave the hospital with empty arms. "The hardest thing to do was walk out of that hospital without her" stated one participant. The participant, who opted for perinatal hospice, shared that the family had not planned for the funeral despite planning everything else so carefully.

Surrendering the body of the baby to personnel from the funeral home is hard for the parent when the baby dies at home. The parent wants to keep the baby at home among family. Allowing the funeral home personnel to take the baby means the process of squeezing a lifetime into a moment is over. One participant stated, "I felt jealous, they were going to get to be the last people to hold her”. Another kept the baby at home for an additional 24 hours prior to taking the baby to the funeral home. "The funeral home came and got her. I wish I would have held her longer" was the desire voiced by a participant who really only got to hold the baby after death.

The burial or cremation of the baby's body is a final physical separation from the parent. The baby is no longer readily available to the parent. It means that the parent will not ever be able to hold the baby again. 
The funeral or memorial service is part of the final physical separation. This service may provide the parent with validation that the baby's life had meaning. Attendance at the funeral or memorial services by family, friends and the health care professionals who cared for the parent and/or baby is part of this validation. The parent appreciates the presence and support of others. This presence seems to validate that the baby was valued even though here for a short time.

Some participants discussed the service held after the baby’s death. One stated, "I always hated wakes and funerals, but I needed it. I could feel his coldness and smell of chemicals and let go of that little shell. He was not there”. A couple of participants talked about how much it meant to have nurses who cared for her and/or the baby come to the funeral services. One found it remarkable that people from church, who were previously unknown, came to the baby’s funeral.

Saying goodbye is a very sensory experience. The parent holds and touches the baby prior to the first and second separation. Some participants dressed the baby in every outfit prior to that first separation. Others bathed, took pictures and held the baby. One participant shared sniffing the baby from head to toe after death in order to remember the baby’s smell

Saying goodbye is a paradox. The parent goes through the motions of saying goodbye; however the deceased baby never totally leaves. The baby lives forever in the parent's memories and heart. Additionally, parents hang on to and/or display items related to the baby such as pictures, blankets, and plaster footprints. These physical reminders are very present even if the baby is not. One participant stated, "I still think I am their mom and they are still my daughters". Yet another participant asserted, “C. is a very real presence in our home”. 


\section{Seeking Meaning in the Baby’s Life}

Seeking meaning in the baby's life is a process that occurs during the going on stage. The parent tries to make sense of the loss by seeking meaning in the baby's life. The baby was here for a reason. The parents talk about the baby impacting others and making a difference in the world, even if here for a short while. Therefore the baby's life had a purpose. This effort seems rooted in some faith in the order of the universe. Something good has to come out of something so awful.

One way a parent seeks meaning in the baby's life is through crediting the baby with contributions of the personal growth of the parent and others. While going through this life and death experience with the baby, the parent may discover or develop personal skills. These skills enable the parent to manage and cope with the life and death situation. The parent may hear from others that the baby contributed to personal growth of that person. Participants discussed becoming stronger or having more patience during and after the experience. One mother stated “Every day I did something I could not have done before. He made me a better person”. One shared that the baby brought a woman to God.

Seeking meaning in the baby's life also involves attributing changes in the institutions in which the baby received care as evidence of the baby's impact. Somehow the baby made a difference in the hospital or hospice. Creation of a lounge for parents of NICU patients occurred after the death of one baby. The lounge was named after the baby. Another baby was an inpatient hospice’s first pediatric patient. After that baby's death, the hospice added more pediatric beds. The parents attributed that change to their baby. A nurse shared with a participant that the relationship between a physician and nurses improved after working together to care for 
the dying baby of the participant. Figure 6 depicts the process of seeking meaning in the baby's life and the property of helping others.

Figure 6: Seeking Meaning in the Baby’s Life: A process with 1 property

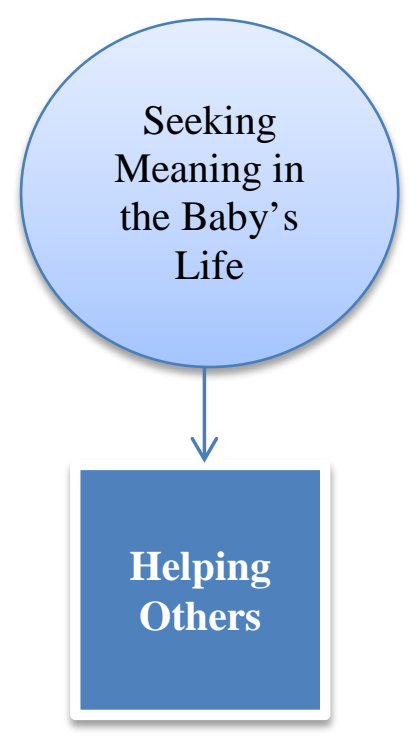

Helping others. An element of seeking meaning in the baby's life is by the parent helping others. Through helping others, the parent ensures that the baby's life was not meaningless. Helping others takes many forms. The parent may volunteer to serve as a personal contact should a person in the area deliver a baby with the same condition or another one that is life-limiting. Making monetary contributions to hospitals, hospices or to foundations specific to the disorder that took the baby's life is another means of helping others.

The internet is a powerful and common outlet for helping others. The parent may develop a personal web site to help other bereaved parents. Organizations, such as the Trisomy 13 Foundation, have web sites to educate and assist parents of babies with certain rare disorders. 
The parent may become active on the private sharing areas of a site to help other parents who are going through similar challenges.

One participant discussed overseeing a fund that helps NICU parents with small expenses such as a tank of gas or food. Another has a web site devoted to helping other parents who experienced the death of a baby. This participant also sent little name bracelets to bereaved parents. Participants also discussed being active on web sites specific to the baby's condition; on these sites the participants were able to help others experiencing a similar situation. Publication of a book about the baby's life was the means by which one participant sought to help others.

Helping others is also a paradox. Parents find that helping others helps them to heal. The participant with the web site stated that the outreach helped her heal, as did the participants who were active on disorder specific websites. The participant who wrote the book to help others stated that the experience assisted in the healing process.

\section{Keeping the Baby's Memory Alive}

Going on also includes keeping the baby’s memory alive. Parents do this by telling their story and keeping mementos. These two properties make the baby's life real. Keeping the baby's memory alive is paradoxical for the parent. Remembering the baby is both painful and joyful. Parents both smile and cry while telling their story and sharing mementos. It is as if the parents are afraid that the baby will be forgotten and that all the pain was for nothing. See figure 7 for a depiction of the process of keeping the baby's memory alive, the property of telling their story with sub-properties of reciting the facts and feeling silenced, and the property of keeping mementos. 
Figure 7: Keeping the Baby’s Memory Alive: A process with 2 properties and 2 sub-properties

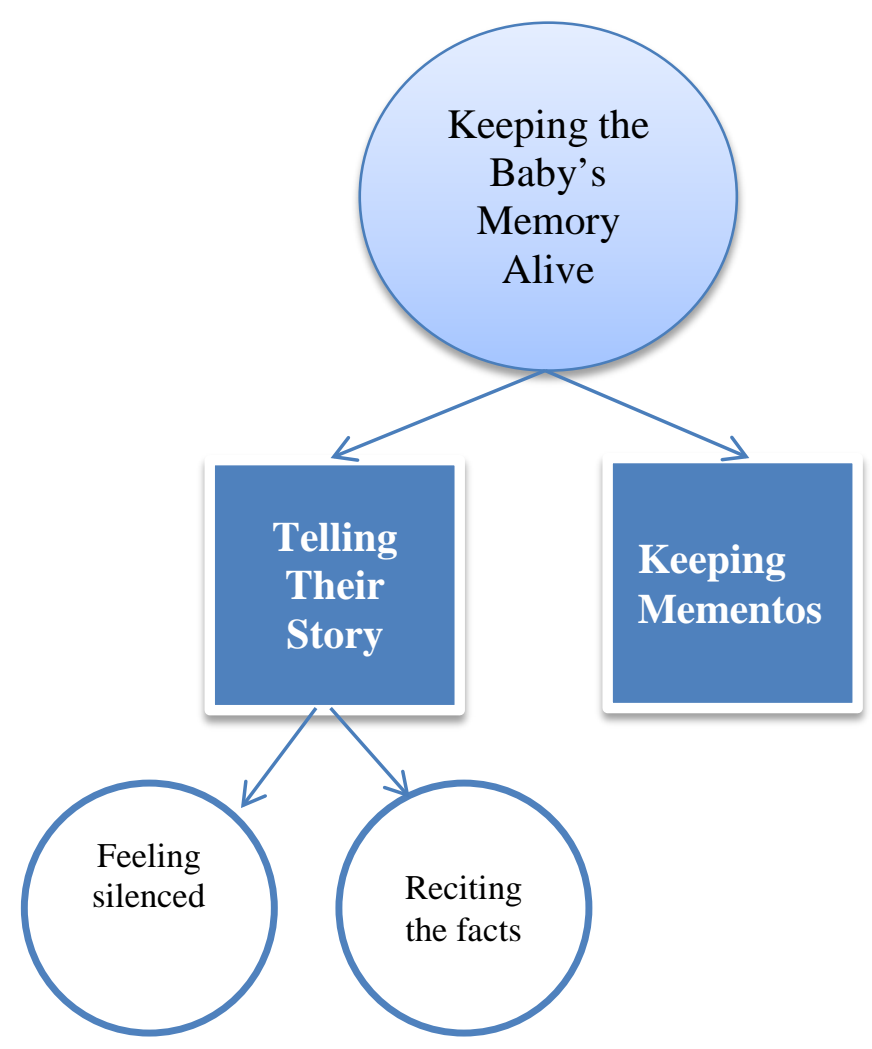

Telling their story. One property of keeping the baby's memory alive is telling their story. The parent wants to tell the story of the life and death of their baby. The baby's life is validated if someone knows the baby’s story. However, telling their story brings both pain and joy to the parent. Parents seem willing to experience the pain because the alternative is to forget, which is not possible. So they tell their story. All participants were very willing to share their story. One stated "I have been looking forward to this all day". Another wrote and published a book about the life and death of the baby.

Reciting the facts. Parents want to tell their story, but doing so is painful. Many resort to reciting the facts when telling their story. Reciting the facts involves presenting elements of their experience in very clinical terms. This method of sharing seems to protect the parent from 
painful feelings. Participants shared very specific clinical aspects of their and the baby's care such as how many milliliters (ml’s) of fluid was drawn off the baby's head or the types of surgery performed.

Feeling silenced. Parents want to tell their story. However, factors exist that make sharing a challenging proposition. The death of a baby is, at best, an uncomfortable subject and practically taboo in this society. Well-meaning family and friends fear that talking about the baby will upset the bereaved parent. Family and friends listen to the story about the baby, but only for so long. The bereaved parent gets the message that others do not want to hear about the baby and feels silenced. Feeling silenced is paradoxical to telling their story.

Many participants discussed feeling silenced when wanting to talk about their dead baby. One participant who stated "I just love talking about N." also said "No one else wants to hear me talk about her anymore". Another participant said "It does not upset me to talk about my dead child, but it can clear a room. Our friends all know about J. Nothing you can say can make it Ok. People are scared to talk about it”. "I was not going to sweep him under the rug” was the assertion of another participant.

Keeping mementos. The other way to keep the baby's memory alive is through keeping mementos. A memento is an item(s) that reminds the parent of the baby and takes many forms. Pictures of the deceased baby are the most common keepsake. The parent may display the photo of the deceased baby in the family home. Photos may also be in a scrapbook created by or for the parent. Family photos shot during creating memories property of squeezing a lifetime into a moment may be part of the scrapbook. The scrapbook may also contain hand/foot prints of the baby or a lock of the baby’s hair. Other mementos include clothing worn by the baby, blankets, a casting of the baby's hands and feet, hand crafted items made by family and friends, and even 
medical equipment such as the baby's blood pressure cuff. These mementos symbolize the reality of the baby's existence. Keeping mementos like saying goodbye is a sensory experience. If the parent touches, sees, or smells something that belonged to the baby, the baby existed.

All participants had something that belonged to the baby. Some had boxes full of mementos. One participant lost everything that belonged to the baby in a house fire except for a footprint card that serendipitously happened to be in a book in her office. Most participants proudly displayed pictures of the deceased baby along with pictures of the living children on the wall of their homes. In one family, the little boys wrapped themselves up in their deceased sisters' blankets. A couple participants reported opening a plastic bag which contained clothing worn by the deceased baby in order to smell the baby.

Keeping mementos is also paradoxical for the parents. The presence of items that belonged to the baby validates the baby's reality. However, those same mementos also reinforce the fact that the baby cannot use the items because he/she is no longer here. So mementos are both comforting and painful. One participant stated "I love to talk about her but cannot go through her things".

\section{Living Life}

The death of a baby does not mean that life stops. Life will never be the same but the parent must go on living life. Surviving and subsequent children, marriages, relationships, careers and other elements of life require the attention of the bereaved parent. Embedded in the process of living life are two properties, having another baby and getting professional help. Figure 8 depicts the process of living life and the two properties. 
Figure 8: Living Life: A process with 2 properties

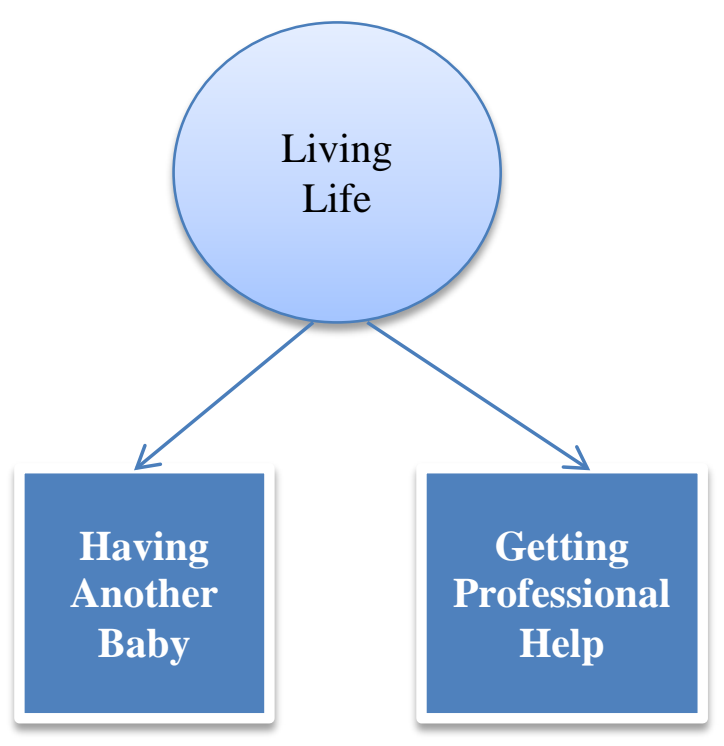

The bereaved parent may not want to get out of bed after the death of the baby. Feelings of sadness, guilt, and grief sap the energy of the parent. But the surviving children have needs that must be met. These needs are a powerful force that impels the bereaved parent to get out of bed each day. Several participants described not wanting to get out of bed after the baby died. However, the needs of the living children were the impetus to do so. "My $3 \frac{112}{2}$ year old was my saving grace. I do not know what I would have done if I did not have to get up every day to take care of him" said one participant. Another participant said "I have to keep going because of my kids”. Others made similar statements.

The baby's illness and subsequent death is a significant stressor. It has to impact the parents' relationships and those relationships with others. However, few of the participants talked about the relationship with the husband or significant other. One talked about the anger felt by her husband and reminded him of the need to lean on each other. Most of the participants 
were still in the marriage into which the baby was born. Only one participant was divorced from the deceased baby's father. The unmarried couple remained friends, but not romantically involved.

The other relationships in the parent's life are impacted by the baby's life and death. Family and friends immediately gather to provide help and support to the parent undergoing the birth, brief life and death of the baby. However, that help and support may have a time limit as discussed in feeling silenced. New relationships also develop during this time especially if the parent becomes involved with other parents on the private sharing area of web sites specific to the baby's condition. Many of the participants talked about how supportive and helpful family and friends were during the immediate crisis of the baby's diagnosis and subsequent death. Also shared was the discomfort of those same people when the participant still wanted to talk about the baby. The relationships developed on the web sites were valuable to some of the participants.

Going back to work may also help the parent get out of bed in the morning. Work may serve to divert attention to something other than the loss. One participant shared "I wanted to come back to work right away, but the doctor made me wait until 6 weeks. I was fine when I was busy, but when I stopped I was a mess”. Another participant, who worked with special needs children under the age of three, was unable to work after the prenatal diagnosis of a lifelimiting congenital anomaly. After the baby’s death, this participant was able to return to work. However, another participant, who worked as a pediatric nurse, ultimately left pediatrics and nursing a couple years after the death of the baby.

Having another baby. A major concern of living life is the decision about having another baby. Having another baby is a major risk for these parents. There is the chance that the 
life-limiting congenital anomaly will occur in a subsequent baby. Even a miniscule likelihood of recurrence of the life-limiting anomaly is too much of a risk for some parents. These parents decide to never try to get pregnant again.

Other parents want to have another baby, but feel fear and trepidation. Genetic counseling is an option some bereaved parents pursue prior to conception. Extensive prenatal testing such as chorionic villus sampling or serial ultrasound are also options for the parents. Pregnancy is no longer assumed to result in the birth of a healthy baby. Living in innocence is forever lost for these parents.

Participants fell on both sides of this decision. One shared "I will never have another child. They don't know enough about teratoma and I won't put another child through that. I won't go through that, it would kill me”. Some participants wanted to get pregnant right away but were advised to wait at least six months. A surprise pregnancy was discovered by one participant as her baby was getting worked up and diagnosed with a life-limiting congenital anomaly. One participant had a second baby with the same rare congenital heart defect as the baby who had died. Despite this, she chose to get pregnant again after the second death. Yet another participant explored getting pregnant through embryo adoption.

Those participants who chose to get pregnant again all had extensive prenatal testing. This testing occurred despite many stating that elective termination was not an option, a paradox. The participant with a second baby with a rare heart defect made different choices for the care of the baby than that for the first baby. Hospice care was chosen instead.

Having another baby for these parents is not replacing the deceased baby. Rather the new baby is a validation that life is good. One participant stated “J. brought laughter back into our home”. 
Getting professional help. The death of a baby is a major negative life event. So it is not surprising that a parent may require professional help to deal with the loss. Concerns about surviving children may also impel the parent to seek the input of a professional. That professional help may take the form of pastoral care, bereavement or other mental health counseling.

The professional serves as a safe person with whom to talk about the baby since the parent feels silenced. The parent may be more willing to share feelings with that professional than with family or friends. Should the parent experience depression, anxiety or other treatable conditions, the licensed mental health professional can prescribe medications. Talking to a pastor can help the parent address any spiritual concerns and questions.

Many of the participants discussed talking to their pastor. Those talks with the pastor provided comfort. One participant sought the services of a counselor once and felt relief. Others participants were still in therapy and on antidepressants and other medications for depression and/or anxiety.

Another professional from whom a parent will seek help is a genetic counselor. In some cases the parent is a carrier for an anomaly, but in others the genetic aberrancy just occurs randomly. This professional can provide some guidance about the likelihood of a specific congenital anomaly recurring. The parent can then weigh options concerning another pregnancy. A couple participants did share working with a genetic counselor.

The process of getting professional help is paradoxical with the process of helping others. Here the parent receives help from others rather than giving help. Both are means for healing. 


\section{Conceptual Hypotheses}

Glaser (1998) states “a substantive theory is an integrated set of conceptual hypotheses that account for much of the behavior seen in the substantive area” (p.3). The conceptual hypotheses for this study include the following:

1. The stage of living in innocence ends with the cutting point of getting the bad news.

2. The stage of being a good mom/dad in the new reality begins after the cutting point of getting the bad news.

3. Seeking answers is the first process in the stage of being a good mom/dad in the new reality.

4. Maintaining hope, being separated from the baby, and making decisions are properties that occur in the process of seeking answers within the stage of being a good mom/dad in the new reality.

5. Squeezing a lifetime into a moment and letting go are processes that occur after and iteratively with the process of seeking answers within the stage of being a good mom/dad in the new reality.

6. Keeping vigil, creating memories and seeking normalcy are properties that occur in the process of squeezing a lifetime into a moment within the stage of being a good mom/dad in the new reality.

7. Being alone with the baby and taking the baby home to die are sub-properties that occur in the property seeking normalcy which is in the process of squeezing a lifetime into a moment within the stage of being a good mom/dad in the new reality.

8. The stage of being a good mom/dad in the new reality ends with the cutting point of the death of the baby. 
9. The stage of going on begins with the cutting point of the death of the baby.

10. The process of saying goodbye to the baby occurs first in the stage of going on.

11. The processes of seeking meaning in the baby's life, keeping the baby's memory alive, and living life are processes that occur after and iteratively with the process of saying goodbye to the baby within the stage of going on.

12. Helping others is a property of the process of seeking meaning in the baby's life within the stage of going on.

13. Telling their story and keeping mementos are properties of the process of keeping the baby's memory alive within the stage of going on.

14. Feeling silenced and reciting the facts are sub-properties of the property of telling their story in the process of keeping the baby's memory alive within the stage of going on.

15. Having another baby and getting professional help are properties of the process of living life within the stage of going on.

\section{Summary}

Parents living within the paradoxes of infant death from life-limiting anomaly is a new grounded theory that emerged out of the stories of bereaved parents whose baby died of a lifelimiting congenital anomaly. Paradox is the core category of this study. In this study, paradox was defined as "something (as a human being, phenomenon, state of affairs, or action) with seemingly contradictory qualities or phases” (Paradox, 1993, p 1636.). Being the parent of a new baby who is going to die while still so new to life is the first paradox of the theory. From this first paradox others paradoxes arise. Within the theory of living within paradox there are many paradoxes which follow: 
1. The parent maintains hope in the midst of a hopeless situation.

2. The parent seeks answers to save the baby which leads to separation from the baby.

3. The parent does not feel like a mom/dad despite acting as a mom/dad.

4. The parent thinks about death at the moment of birth.

5. The parent attempts to squeeze a lifetime into a moment.

6. Memories of the baby evoke both sadness and joy.

7. The parent seeks answers to save the baby and lets go.

8. The parent provides sustenance to a baby that is unsustainable.

9. The memory of the baby lasts far longer than the baby was on earth.

10. The parent tries to create positive memories of a time that is painful.

11. The parent shares the baby with others but wants to be alone with the baby.

12. The parent attempts to create a sense of order in an out-of control situation.

13. The parent seeks normalcy in an abnormal situation.

14. The parent desires being alone with the baby in the midst of the busyness of the NICU.

15. The parent takes a newborn home to die.

16. The baby needs more care than a normal newborn in some ways and less care in other ways.

17. The parent maintains hope and lets go.

18. The parent wants the baby to live but also wants the suffering to stop.

19. The parent feels sadness and relief at the baby’s death.

20. The parent says goodbye to a baby that never really leaves.

21. The parents finds healing in helping others.

22. The parent feels silenced but wants to tell their story. 
23. Mementos provide comfort and cause pain.

24. The parent goes on living life despite not wanting to face life.

25. The parent seeks professional help but helps others.

The theory of living within paradoxes consists of three stages with two cutting points. During the stage of living in innocence the expectant/new parent enjoy the pregnancy or new baby, unaware that life is about to take a dramatic turn. Breaking the bad news is the cutting point that ends the stage of living in innocence and forces the parent into the stage of being a good mom/dad in the new reality. During the stage of being a good mom/dad in the new reality the parent is seeking answers which often results in the need for making decisions; maintaining hope and being separated from the baby occur too. Squeezing a lifetime into a moment also happens during being a good mom/dad in the new reality. The parent demonstrates squeezing a lifetime into a moment by keeping vigil at the baby's bedside, creating memories of the limited time with the baby, and seeking normalcy in this abnormal situation. While seeking normalcy, the parent craves being alone with the baby and makes the decision about taking the baby home to die. Letting go happens as the parent realizes the inevitability of death. Squeezing a lifetime into a moment comes to an end at the death of the baby, the second cutting point. The parent moves into the stage of going on where the parent experiences saying goodbye to the baby. Helping others is one means by which the parent is seeking meaning in the baby's life. Keeping the baby's memory alive is vital to the parent and happens by keeping mementos and telling their story. While telling their story, the parent may do so by reciting the facts and may experience feeling silenced when others do not want to listen. Finally the parent must go on living life which may require decisions about having another baby and may include seeking professional help. 


\section{CHAPTER FIVE: DISCUSSION}

The grounded theory of Parents Living within the Paradoxes of Infant Death from LifeLimiting Anomaly (living within paradoxes) is a new theory. This theory describes the how a parent goes through experience of the pregnancy, birth, diagnosis, life, and death of an infant with a life-limiting congenital anomaly. In this chapter, the investigator presents the following: (a) a summary of the theory, (b) a comparison of the grounded theory of living within paradoxes with the published literature, (c) a critique of the theory, and (d) implications for nursing education and practice, and research.

\section{Summary of Living within Paradoxes}

The grounded theory of living within paradoxes consists of three stages and two cutting points. In the first stage of living within paradoxes, the parent-to-be/parent is living in innocence. Living in innocence abruptly ends with the cutting point of getting the bad news. Getting the bad news occurs when the parent learns that there is a major health issue with the baby. This cutting point may happen prenatally or after birth.

The stage of being a good mom/dad in the new reality starts after getting the bad news. During the stage of being a good mom/dad in the new reality, the following processes occur: seeking answers, squeezing a lifetime into a moment, and letting go. These processes may be sequential or iterative.

A second cutting point occurs at the death of the baby. Going on is the stage that follows the death of the baby. Saying goodbye, seeking meaning in the baby's life, keeping his/her memory alive, and living life are processes that take place during the going on stage. As with the second stage, the processes may occur simultaneously and in sequential or iterative manner. 


\section{Comparison of the Theory with the Literature}

It is customary in grounded theory research to search out relevant theoretical and empirical literature during the theorizing process. Glaser (1978) suggests that a review of the literature only occur after the theory is "sufficiently grounded and developed" (p. 31). This review allows the researcher to "relate the theory to the literature through the integration of ideas” (Glaser, 1978, p. 31). Glaser also suggests that reading of the extant literature allows the theorist to have increased theoretical sensitivity. In this section, this author will examine and analyze the empirical and theoretical literature for relevance to living within paradoxes.

\section{Empirical Research Literature}

There is little research about palliative and end-of-life care for neonates in general and even less for infants with life-limiting congenital anomalies specifically. Nevertheless, it is important to examine how that research compares to the grounded theory of living within paradoxes. This author refers the reader back to the literature reviewed in chapter 2 . In addition to the original literature review, it is customary in grounded theory research to search out literature that is found to be relevant when gathering data and theorizing progress. Therefore, as is appropriate to the method, this chapter also presents pertinent literature that was not previously reviewed. A discussion of how the research literature relates to the grounded theory of living within paradoxes follows. In the subsequent sections, this author placed the stages, processes, and properties of the theory in italics to distinguish those aspects of the theory from the findings of other authors' work.

Withdrawal of Life Support. The literature about withdrawal of life support exhibits some relevance to the theory of living within paradoxes. Many of the participants of this study had made the decision for withdrawal of life support for their baby. Other participants chose not 
to initiate these measures at all (see chapter 4). The relevance appears in the stage of being a good mom/dad in a new reality. Parents, in partnership with the health care team, are the ones making decisions about withdrawal of life support. The decision to withdraw life support often comes as maintaining hope wanes and letting go takes place. Conversely, the researchers of the withdrawal of life support studies used retrospective chart audit to look at documentation only. So while there may be relevance in withdrawal of life support and the theory, it is difficult to make that assertion based on findings from a chart audit.

Many of the findings of an international study by Williams et al. (2009), not included in the original literature review because of the strict inclusion criteria, supports living within paradoxes. Williams et al. (2009) interviewed 11 sets of bereaved parents as a first step toward development of a tool to measure end-of-life care after withdrawal of life-sustaining treatment (WLST) in a NICU. The parents’ views guided the development of survey questions to be asked of health professionals. Survey questions were organized into the following content domains: communication, quality of care, quality of life, shared decision-making, WLST process, and bereavement care (Williams et al., 2009). Many of the items correlate with aspects of the theory of living within paradoxes. Many of the communication items (give parents the right amount of hope and information giving) correlate with the property of maintaining hope and the process of seeking answers. The shared decision-making domain is identical to making decisions in the theory. Almost all of the items in WLST process domain are relevant to the process of squeezing a lifetime into a moment. Bereavement care incorporates many aspects of the stage of going on. Items in the quality of care and quality of life domains are very health care worker focused and do not correlate with the theory. The infants of the Williams et al. study participants died of prematurity as well as life-limiting congenital anomalies after non-initiation of life-sustaining 
treatment or WLST. While there are some differences between the causes of death, the deaths were expected in both studies. It is interesting that the tool will measure health care professionals’ perceptions of end-of-life care rather than those of the parents.

Palliative/End-of-life care. Living within paradoxes is central to the subject of palliative and end-of-life care for dying neonates and infants. It is unknown if any of the participants had a palliative care consult. This investigator did not specifically ask about this. However, over half of the infants of the participants of this current study received hospice care $(n=7)$. This number contradicts other authors who found or estimated the percentage to be between 9-20\% (Friebert and Huff, 2009; Last Acts, 2002; Leuthner et al., 2004; Zwerdling, 2000). Almost half of the infant in this current study died at home $(n=5)$. That is contrary to the findings of Leuthner, Boldt and Kirby (2004) who found that only $4 \%$ of the infants died at home. These findings about hospice care and dying at home in this study probably do not reflect a great increase in the number of infants dying at home. Rather the number reflects this investigator’s source of participants which was from a hospice.

A study by Widger and Picot (2008) supports the theory of living within paradoxes even though the inclusion criteria of the two studies were different. Widger and Picot sought bereaved parents' perception of the quality of end-of-life care for their baby or child. Thirty-eight families were interviewed via telephone with a tool developed by the investigators, no data was provided about the reliability of the tool. Widger and Picot reported that the parents' overall satisfaction with end-of-life care was $84.6 \%(n=33)$. Almost all of the bereaved parents (95\%) reported having ample opportunity to ask questions and have information repeated. Some parents reported the following communication issues: not getting enough information $(n=7)$, not knowing who to ask for help ( $\mathrm{n}=6)$, and difficulty understanding the health professional $(\mathrm{n}=10)$ 
(Widger \& Picot, 2008). In the area of relationships, almost all of the parents reported being treated with courtesy and compassion $(n=36)$. All parents who had a baby die wanted and received mementos, conversely not all parents who had a child die did. The communication issues mentioned by Widger and Picot coincide with the process of seeking answers and property of making decisions in living within paradoxes. The property of creating memories and process of keeping mementos of living within paradoxes are congruent with the parents wanting mementos in the Widger and Picot study. The Widger and Picot study included bereaved parents of children older than 15 months with a variety of conditions including stillbirth. Therefore, there is limited congruence between the participants of this current study and of the Widger and Picot study.

Findings of a study by Branchett and Stretton (2012) also support the theory of living within paradoxes. The two British investigators asked 57 participants of an online support group for stillbirth and neonatal death to answer two questions. The questions were related to (a) telling health professionals about the experience of the loss of a baby or experience of palliative care and (b) what was 'good' about the palliative care received during that experience (Branchett \& Stretton, 2012). Eight themes arose from data analysis and indicated what aspects of care were most important to the bereaved parents. Creating memories, empathy, time and space, practical help and understanding, sensitivity, communication with parents, accurate record keeping and communication, in utero, and support afterwards comprised those themes (Branchett \& Stretton, 2012). The first theme creating memories mirrors the process of creating memories in the theory. Discussion of the theme of creating memories included the importance of mementos and normalcy which coincide with the properties of keeping mementos and seeking normalcy in living within paradoxes respectively. Aspects of the need for time and space are 
similar to the sub-property of being alone with the baby. The need for information in seeking answers was implicit in the theme of communication with parents. Finally, the property of living life and sub-property of getting professional help corresponds with the theme of support afterwards. The other themes in the Branchett and Stretton study do not correspond to aspects of the theory of living within paradoxes. Rather, the themes are aspects of palliative and end-of-life care identified as important by the bereaved parents. In contrast this theory describes how parents go through the process of having an infant with a life-limiting congenital anomaly.

Parents. The findings of the study of bereaved parents by Brosig et al. (2007) support the grounded theory living within paradoxes. Brosig et al. described strategies bereaved parents used to cope: seeking support from family and/or a bereavement support groups, focusing on the surviving children, memorializing the dead infant in some manner, turning to God, and giving back by monetary donations or volunteering personal time to help other children. These strategies are similar to the processes and properties in the stage of going on in the theory. Seeking support in the Brosig et al. study correlates with the process of seeking support in the theory. The process of seeking meaning in the baby's life receives support from the strategies of memorializing the dead baby and giving back. Keeping the baby's memory alive also is congruent with memorializing the dead baby. The process of helping others is practically identical to giving back.

Many of the stages, processes, and properties of the grounded theory of living within paradoxes mirror categories and themes applied to parent's bereavement experiences by Tan et al. (2011). The process of seeking answers and properties of maintaining hope and making decisions are similar to the themes of entertaining possible outcomes and decisions they can live with, themes of the Tan et al study. Memory making with themes of remembering time spent 
together and physical memory making supports many aspects of the theory: creating memories, being alone with the baby, seeking normalcy, and keeping the baby's memory alive. The processes of living life and helping others in the theory correlate with the categories of wide network of support and altruism respectively. The Tan et al. study begins at diagnosis which may be before delivery or after birth. Conversely, the grounded theory of living within paradoxes expands beyond the Tan et al. study by including the time before getting the bad news when everything was fine and the parent was living in innocence.

One finds correlations between the themes of the Lathrop and VandeVusse study of bereaved mothers and elements of living within paradoxes. Keeping the baby's memory alive, keeping mementos, and saying goodbye to the baby correlate with the theme of continuity. Lathrop and VandeVusse (2011) reported that the mothers expressed “continuing feelings of love” (p. 25) for the baby. In contrast, only one parent stated overtly about loving the baby in this current study. The investigator in the present study made a point of avoiding the term love during interviews in an attempt to allow participants to voice their experience from their own perspective without the burden of preconceived notions. Thus, feelings of love were not voiced by the participants of this study despite demonstrating loving behaviors during the short life of their babies. Personal growth is integral to both the theme of evolving changes and the process of seeking meaning in the baby's life. The theme of transient phases parallels the cutting points of getting the bad news and the death of the baby as well as the property of saying goodbye in the theory. There are many correlates in the properties in the stage of going on and the theme of connecting past to present. Lathrop and VandeVusse also used the word paradox to describe the mothers' conflicting feelings and thoughts over time. The participants of the two studies differ in two important ways. All the participants of the Lathrop and VandeVusse study had a prenatal 
diagnosis of lethal anomaly and received perinatal palliative/hospice care during pregnancy.

Whereas, only some of the participants had a prenatal diagnosis and only one participant of this study had perinatal hospice care.

Living within paradoxes receives support from the results of a study by Black and Sandelowski (2010) which examined the personal growth of men (n-6) and women $(n=12)$ after diagnosis of severe fetal anomaly. Black and Sandelowski analyzed ethnographic interview data using the Posttraumatic Growth Inventory (PTGI). Organization of the dimensions of change occurred in the following manner: (a) relating to others, (b) new possibilities, (c) spiritual development, (d) appreciation of life, and (e) personal strength (Black \& Sandelowski, 2010). Black and Sandelowski reported that most of the participants (n-18) reported positive growth; the rest reported negative change $(n=3)$, a mixture of positive and negative $(n-1)$ or no change $(n=2)$. Some of those changes are congruent with aspects of the theory of living within paradoxes. Helping others in the theory of living within paradoxes has some congruence with the change of having more compassion for strangers. Both positive spiritual changes (seeking meaning from the experience) and growth in personal strength closely align with the process of seeking meaning in the baby's life. Future parenting, an aspect of new possibilities, is almost identical to the sub-property of having another baby. This author finds no obvious correlate of the domain of appreciation for life in the theory of living within paradoxes. There was a significant difference in the participants of the two studies. Some of the participants in the Black and Sandelowski study had terminated the pregnancy upon diagnosis whereas none of this current study had.

The grounded theory of living within paradoxes is an important addition to the literature because this theory more comprehensively describes the experience of bereaved parents who 
experience the death of a baby with life-limiting congenital anomalies than any of the reviewed studies. Results of many of the reviewed studies had components that supported the theory of living within paradoxes. However, most of the studies reviewed only looked at the bereavement experience of the parent with the exception of one study that looked at the time from diagnosis to birth. In contrast, this current study explored the entire experience of the parent from pregnancy to beyond the death of the baby.

\section{Theoretical Literature}

Once review and analysis of the empirical literature occurred, it was time for the review and analysis of the theoretical literature relevant to living within paradoxes. This author faced a quandary when looking at the theoretical literature related to the experience of parents whose neonate/infant died from a life-limiting congenital anomaly. Theoretical literature about death and dying, bereavement, and/or parenting all could conceivably be relevant. Paradox was also an obvious option. Therefore, a smattering of all may be found in this section.

Death and Dying. The death and dying theories selected with which to compare the theory of living within paradoxes are classics. As the original grounded theories, the theories of awareness of dying and time for dying by Glaser and Straus were obvious choices for inclusion in this discussion. The five stage of dying by Kubler-Ross became a much cited and debated theory. These three theories and the theory of living within paradoxes share the characteristic of being grounded in the experience of the participants, rather than any preconceived ideas.

Awareness of dying. Glaser and Straus (1965) analyzed the context of awareness of dying in the hospital setting. During this study, researchers observed and interviewed patients, family members, and hospital staff including nurses and doctors. Four contexts of awareness emerged from the analysis of data: closed awareness, suspicion awareness, mutual pretense, or 
open awareness (Glaser \&Strauss, 1965). Glaser and Strauss theorized that the type of awareness present impacts the behaviors of and interactions among hospital personnel, the patient's family, and the patient.

Elements of the theory of living within paradoxes suggest coherence with elements of awareness of dying. Specifically, it seems that open awareness may occur with the cutting point of getting the bad news and continues throughout the stage of being a good mom/dad in a new reality until the cutting point of death of the baby. However, open awareness implies that the patient is aware that he/she is dying. The dying patient in this current study is an infant who does not have the cognitive ability to understand what this means. One may wonder if open awareness is possible in this situation. Conversely, the argument could be made that if one practices family-centered care, then open awareness is possible since the family is the patient or client.

Suspicion awareness is a possibility for that parent who "has a feeling something is wrong” during the stage of living in innocence. Closed awareness could conceivably occur in the stage of living in innocence if a health care provider withheld bad news until the results of testing confirmed the diagnosis of a life-limiting congenital anomaly. However, just the order for special tests may trigger suspicion awareness in the parent. It seems unlikely that mutual pretense could occur in these particular situations in the current health care climate. Therefore, awareness of dying does have limited applicability with the theory of living within paradoxes.

Time for dying. The book “Time for Dying” followed “Awareness of Dying”. In the book, Glaser and Strauss (1968) described perceived trajectories of dying with two properties: time and shape. Dying may occur slowly or rapidly, a function of time. The property of shape may be demonstrated by a sudden decline in health or a more insidious downward course 
characterized by exacerbations and remissions. Glaser and Strauss postulated the notion of "death expectations" or the combination of certainty and time of dying with the following possibilities: "certain death at a known time, certain death at an unknown time, uncertain death but a known time when certainty will be resolved, and uncertain death and an unknown time when the question will be resolved” (p. 8). The interactions of health care personnel, families and the dying patient are impacted by the perceived trajectories and death expectations (Glaser \& Strauss, 1968).

Living within paradoxes is relevant to time for dying in several ways. Time is a critical factor. The parents of infants with life-limiting congenital anomalies want more time. Because the dying trajectories of neonates/infants with life-limiting congenital anomalies vary, some infants die within minutes of birth while other live hours, days, weeks, or months. It is because of the limited time that the parent seeks to squeeze a lifetime into a moment.

Shape is also an element critical to living within paradoxes. A rapid decline severely decreases the time parents spend being alone with the baby, creating memories, and seeking normalcy. Letting go may happen sooner because it is obvious the baby is dying. Conversely, a more insidious course allows for more time in the stage of being a good mom/dad in a new reality and all the process and properties of the stage. Maintaining hope occurs more with the slower decline because the baby has good hours or days and may ultimately lead to more difficulty in letting go.

Finally, death expectations exemplify the characteristics of many life-limiting congenital anomalies. Some life-limiting congenital anomalies are incompatible with life with a certain death at a known time. An uncertain death and an unknown time when the question will be resolved may characterize other life-limiting congenital anomalies, such as some heart defects. 
The death expectation impacts on all aspects of the stage of being a good mom/dad in the new reality, but especially the process of seeking answers and properties of decision making and maintaining hope. Many aspects of living within paradoxes find relevance in time for dying.

Kubler-Ross. Another classic theorist in death and dying is Dr. Elizabeth Kubler-Ross. Kubler-Ross (1969) theorized five stages of death and dying: denial, anger, bargaining, depression and acceptance. Implied in the stages is the idea that the dying person goes through the stages in a unidirectional manner. Like Kubler-Ross’s theory of dying, the grounded theory of living within paradoxes consists of stages. There is an element of unidirectional motion in the stages of living within paradoxes as the parent moves from one stage to the next. However that movement to the next stage only happens because of the occurrence of the cutting points: getting the bad news and death of the baby. The parent has no choice but to move to the next stage. This differs from unknown motivators that cause the dying person to move into the next stage of dying in Kubler-Ross's theory. Some reject the unidirectional aspect of Kubler-Ross’s theory and suggest the dying person passes iteratively through the five stages (Copp, 1998). Within two of the stages of living within paradoxes, a number of processes and properties occur in an iterative manner which contrasts with Kubler-Ross's sequential structure. Finally there exists a major difference between the two theories, that of focus. Kubler-Ross's theory focuses on the experience of the dying person; whereas living within paradoxes focuses on the experience of the parents who have a baby dying of a life-limiting congenital anomaly. Thus, the theory of living within paradoxes has limited applicability with the theory of dying by Kubler-Ross..

Bereavement/Grief. There are many theories about bereavement and grief in the literature. This author chose to compare the theory of living within paradoxes with the bereavement and grief theorists most often cited in the literature. The order of the discussion 
follows the trend in the literature from unidirectional stages/tasks models to a more iterative model.

Lindemann. Lindemann (1944), one of the first modern grief theorists, developed the theory of grieving after the study of individuals bereaved as the result of disasters. Bereaved persons experience the following five phases: (a) somatic disturbances, (b) preoccupation with the image of the deceased, (c) guilt, (d) feelings of hostility or anger, and (e) difficulty in carrying out every day routines (Lindemann, 1944). Lindemann suggested that psychological coping with the loss required that the bereaved person become emotionally detached from the deceased and adapt to a world without the deceased. Also identified by Lindemann were parameters for normal and pathological grief. Many of those identified as pathological then, are now considered normal aspects of grieving (Buglass, 2010). The phases of Lindemann seem to be more symptoms than phases of grief to this author. Therefore it is impossible to compare the symptoms of grief with the theory of living within paradoxes.

Parkes. The theory of grieving by Parkes (1998) consists of four phases: numbness, pining, disorganization and despair, and reorganization. Numbness occurs shortly the death of a loved one as the newly bereaved person represses feelings of great distress (Parkes, 1998). During pining, the bereaved does the everyday of life but experiences "transient episodes of separation distress", also known as pangs of grief (Parkes, 1998, p.3). Parkes theorized that the bereaved person repeatedly reviews the events surrounding the loss during the phase of disorganization and despair. Finally, reorganization occurs as the bereaved person lets go of the attachment to the deceased and reviews and revises their worldview (Parkes, 1998). The four phases do not always occur in a linear manner but may arise at times of significance, such as the anniversary of the death. 
Parkes (1998) noted that the process of grieving includes the conflicting urges (paradox) to look back and look forward during the last two phases of grieving. This paradox is similar to the paradox seen in the processes of saying goodbye to the baby and keeping the baby's memory alive in the stage of going on. Some aspects of Parkes' reorganization bear resemblance to the process of living life. One major difference between the two studies is that the participants of the present study did not seem to let go of their attachment as suggested by Parkes. The iterative nature of the phases of Parkes theory of grieving is similar to the iterative nature for the processes within the stage of going on in living within paradoxes. The theory of grieving is somewhat congruent with aspects of the living within paradoxes, particularly the stage of going on.

Worden. Worden (2002) proposed four tasks that the bereaved person must work through for resolution of grief to occur. Instead of stages espoused by earlier theorists, Worden used tasks to indicate the active nature of grief work. Those four tasks occur in a sequential manner and include the following: (a) accept the reality of the loss, (b) work through and experience the pain of grief, (c) adjust to an environment without the deceased person, and (d) withdraw emotionally from or relocate the deceased and move on (Worden, 2002).

Worden (2002) suggested that the bereaved must acknowledge the loss at both an intellectual and emotional level for completion of the first task. That first task is particularly congruent with the process of saying goodbye to the baby in the stage of going on. According to Worden, the second task of working through the pain means just that, working through the pain of grief as well as any other feelings related to the loss such as anxiety or guilt. This task is inherent in all the processes and properties of the stage of going on. Worden suggests that the third task involves external, internal and spiritual adjustments; all of which one finds in the stage 
of going on. The last task requires the bereaved person to move on with life while maintaining emotional bonds to the deceased (Worden, 2002). Worden’s paradoxical notion of moving on and staying connected is very congruent with the notion of paradox in the theory of living within paradoxes. Worden's grief model is consistent with aspects of the last stage of the theory of living within paradoxes.

Dual Process Model. Stroebe and Schut (2010) described the dual process model of coping with bereavement (DPM) as "a taxonomy to describe ways that people come to terms with the loss of a loved one” (p. 277). Within the DPM are two opposing stressor, lossorientation and restoration-orientation. When a bereaved person focuses on, appraises or processes some aspect of the loss, that person is experiencing loss-orientation (Stroebe \& Schut, 2010). Conversely, focus on stressors to reorient self in a world without the deceased is restoration-orientation (Stroebe \& Schut, 2010). Stroebe and Schut noted that the bereaved person either attends to (confronts) or avoids the two stressors in a dynamic manner (oscillation) which changes over time. Coping with bereavement occurs in the process of attending to or avoiding the two stressors.

Living within paradoxes and DPM share a most obvious concept, that of paradox. The multiple paradoxes of living within paradoxes have already been discussed. Stroebe and Schut (2010) suggested that paradox presents in the DPM as the bereaved person oscillates between the opposing forces of attending to and avoidance. The DPM is congruent with the stage of going on in living within paradoxes. Oscillation between attending to and avoidance is similar to the iterative occurrence of the processes in the stage of going on. The process of keeping the baby's memory alive and properties of telling their story and keeping mementos are consistent with the loss-orientation of the DPM. Congruence exists in the restoration-orientation of the DPM with 
seeking meaning in the baby's life and living life. Therefore, the two theories do share some commonalities.

Parenting. The current study examines a situation in which bereavement occurs.

However, this study is about more than bereavement. The parent is being the mom or dad to the baby, even if for a very short time. Therefore it is important to examine some of the theoretical literature related to parenting.

Becoming a mother. The middle range theory of becoming a mother (BAM) was once known as maternal role attainment. Mercer (2004) suggested the name change was needed to fully capture "the continued expansion of self as a mother" (p. 231). Four stages occur as a woman becomes a mother: (a) commitment, attachment, and preparation (pregnancy), (b) acquaintance, learning, and physical restoration (first 2 to 6 weeks following birth), (c) moving toward a new normal ( 2 weeks to 4 months), and (d) achievement of the maternal identity (around 4 months) (Mercer, 2004, p. 231). Many aspects of the environment (family, friends, community and society) influence a woman as she becomes a mother.

Mercer's theory is particularly relevant to the stage of living in innocence. It is during living in innocence that the expectant mother is completing the tasks of commitment, attachment and preparation. Getting the bad news during the prenatal period may alter the tasks of the first stage. Attachment of the woman to the fetus may be impacted. Additionally, preparation changes from getting ready for a healthy baby to making funeral plans. Attainment of the other stages again depends upon the timing of getting the bad news. Should diagnosis of the baby's life-limiting congenital anomaly occur after the baby is four months of age, the mother may fulfill the fourth stage and achieve a maternal identity. Conversely, if the diagnosis of the lifelimiting anomaly and/or death occurs prior to that fourth month, the mother may not achieve 
stage two or three, let alone maternal identify. Becoming a mother has limited congruence with living within paradoxes since BAM is only applicable to females, whereas living within paradoxes applies to both genders.

Safeguarding precarious survival. In the theory of living within paradoxes one finds correlates with some aspects of the constructivist grounded theory of safeguarding precarious survival: parenting children who have life-threatening heart disease. In a study by Rempel and Harrison (2007), parents of children with hypoplastic left heart syndrome exemplified “extraordinary parenting” which manifested in the need to "simultaneously safeguard their child, themselves, and their couple relationship” (p. 827). Involving others, an aspect of safeguarding precarious survival of the child as described by Rempel and Harrison, correlates with the property of living life. The sub-properties of being alone with the baby and taking the baby home to die are similar to elements Rempel and Harrison's strategy of taking charge. Safeguarding survival of self and couple included a strategy of normalizing. Normalizing possesses an obvious similarity to the process of seeking normalcy. The process of maintaining hope resembles trusting in some ways.

Many of the other strategies described by Rempel and Harrison (2007) do not correlate with the grounded theory of living within paradoxes. The strategy of taking charge noted by Rempel and Harrison was not found in the theory of living within paradoxes. In this current study there initially was a similar theme, taking ownership of the baby. However, that theme was not consistent with all of the participants and was thus subsumed into seeking normalcy. One wonders if there may not have been time for this to occur for all of the participants of this study, given the very short lifespan of some of the babies. Rempel and Harrison had many 
strategies related to the dynamics between the parents such as directing their minds, buffering severity, trusting and staying on the same page.

One would expect that there would be differences between the two studies. The infants/children of the parents in the Rempel and Harrison (2007) study were or had been seriously ill, but were still living. In contrast, all of the infants of the participants in this study were all deceased. An additional source of the differences arose from the fact that Rempel and Harrison interviewed couples. This investigator interviewed one couple; the rest of the participants of this study were the mother of the deceased baby. Thus, safeguarding precious survival has limited congruence with the theory of living within paradoxes.

Paradox. Living within paradoxes is the core category of this current study. Paradox is a phenomenon common to all humanity. As a consequence there is a vast amount of literature devoted to this concept. Struggling with paradoxes is the basic social psychological process (BSP) that emerged from a grounded theory study of the spiritual development of women with cancer. Paradox is also a central feature of Parse's theory of humanbecoming. Therefore, it is important to discuss the relevance of struggling with paradoxes and theory of humanbecoming with the theory of living within paradoxes.

Struggling with paradoxes. Tuls Halstead and Hull (2001) used grounded theory to explore the process of spiritual development in women with cancer. Struggling with paradox is a three phase basic social process that emerged in this study. The three phases included: deciphering the meaning of cancer for me, realizing human limitations, and learning to live with uncertainty (Tuls Halstead \& Hull, 2001). The women moved through the phases in a sequential manner, although there was some overlap between phases. It is within each of the phases that one finds the paradoxes. The focus of the paradoxes from each phase follows: 
- Deciphering the meaning of cancer for me: possibility of death, distress, vulnerability and maintaining connection

- Realizing human limitations: confronting death, asking difficult questions, and letting go

- Learning to live with uncertainty: uncertainty, redefining meaning, and identifying spiritual growth. (Tuls Halstead \& Hull, 2001, p. 1534).

“I am alive/does the cancer diagnosis mean that I'm dying?” is an example of a paradox from phase I (Tuls Halstead \& Hull, p. 1537). The authors noted that resolution of the paradoxes may or may not occur. Rather the women learned to live with or let go of the paradoxes.

There are some similarities to the theory of struggling with paradoxes and living within paradoxes. First, the most obvious similarity is the presence of paradox. Both theories consist of 3 unidirectional stages/phases. In the theory of living within paradoxes, movement from one stage to the next was forced upon the parent by the cutting points. Conversely in Tuls Halstead and Hull's struggling with paradoxes, movement from one phase to the next was not always forced upon the woman and there were no cutting points, rather there was overlap between the phases. Both theories do share the process/dimension of letting go. Helping others was a means of redefining meaning in struggling with paradoxes and seeking meaning in the baby's life in living within paradoxes. The most pertinent commonality is the presence of death. In both theories the participants face death. Women in struggling with paradoxes face the possibility of their own death whereas the parent in living within paradoxes experiences the actual death of a baby. So while there are some similarities between the two theories, the reality of death is a major difference.

Parse. The theory of humanbecoming by Parse is a grand nursing theory in which paradox is a significant concept. Parse's theory describes the humanuniverse's process of 
becoming. Evolution of the theory occurred over time in which Parse merged the word human and becoming and human and universe to reinforce the notion of "indivisible co-creation" (Parse, 2007, p. 308). Parse's (2007) theory consists of three principles with four postulates. Those three principles include the following: (a) structuring meaning is the imaging and valuing of languaging, (b) configuring rhythmical patterns of relating is the revealing-concealing and enabling-limiting of connecting-separating, and (c) cotranscending with possibles is the powering and originating of transforming (Parse, 2007, p. 309). Parse asserts that illimitability, paradox, freedom and mystery are the four postulates that "permeate" the three principles of the theory (p. 309). Time and space limitations prevent a full discussion of Parse's theory. Rather this author focuses on paradox in Parse's theory.

As stated above, paradox "permeates the three principles” of the theory (Parse, 2007, p. 309). Parse (2007) defines paradox as "the intricate rhythm expressed as a pattern preference. Paradoxes are not opposites to be reconciled or dilemmas to be overcome but, rather are lived rhythms” (p. 309). Being separated from/being alone with the baby, maintaining hope/letting go, or helping others/receiving help are just a few of the paradoxes in living within paradoxes that one could view as Parse does, as rhythms of living. Conversely, the parent may view being separated from the baby as a major dilemma that needs to be overcome in order to experience being alone with the baby. This author theoretically sees much congruence in paradox as used and defined by Parse and the theory of living within paradoxes. However, at the personal level, it would be hard to see some of these paradoxes as something other than a situation needing reconciliation or a dilemma to overcome. 


\section{Critique of the Theory}

\section{Adequacy}

Glaser (19780 states that the criteria by which to judge any substantive grounded theory are fit, work, relevance and modifiability. Glaser and Strauss wrote about the first three criteria in the book, Discovery of Grounded Theory (Glaser, 1978). Modifiability is a newer addition to grounded theory based on Glaser's experience of using grounded theory over time.

Fit. Fit occurs if the categories of the theory fit the data (Glaser, 1978). The core category, stages, processes and properties of the grounded theory of living within paradoxes all arose from the data, which were the participants' stories. No aspect of the theory was the result of forcing any preconceived ideas of this author into the theory. Rather, the absence of some properties and the appearance of others were a surprise to this author. Thus, the theory meets the criteria of fit.

Work. Work refers to the ability of the theory to explain, predict and interpret what is happening in the area of study (Glaser, 1987). The grounded theory of living within paradoxes allows one to explain and interpret the experience of a parent who experiences the death of a baby from a life-limiting congenital anomaly and is willing to talk about that experience. Additionally, one may make predictions about what behaviors a parent may exhibit while going through this experience. Meeting the criteria of work allows for the use of the theory by health care professionals who provide care to the families of babies with life-limiting congenital anomalies. For example, a nurse in the NICU may make an extra effort to assist the parent in the creation of memories because that nurse anticipates the importance of mementos later to the bereaved parent. 
Relevance. Relevance is the idea that the theory is conceptually about the core concerns of the participants or area of study (Glaser, 1978). All aspects of the grounded theory of living within paradoxes arose from the perceptions and stories of the participants. This author had some preconceived ideas about finding how the parent of a baby with a limited life expectancy attached to and let go of the baby at the same time. However, this particular paradox was not mentioned by the participants. However, letting go was a process the participants mentioned and thus became a part of the theory.

Another preconceived idea was about loving the baby. This author assumed that the parent would talk about how much the baby was loved. The assumption was so strong that the spill question for the pilot study on this same topic was “Tell me about learning to love your baby”. The participants of that pilot study looked perplexed and stated they did not know how to answer that. At that point, this investigator asked the participants to tell their story. That experience resulted in a change to the current spill question. In this current study only one participant overtly said that she loved her baby. None of the other participants talked about loving or bonding with the baby despite displaying behavior indicative of both. Therefore this theory reflects the participants' concerns and meets the criteria of relevance.

Modifiability. Finally, a theory is modifiable if new data provides the impetus to modify the theory. Grounded theories are not verifiable but are rather modifiable to reflect an everchanging world (Glaser, 1978; Glaser, 1998). The theory underwent modification throughout the process of data collection, coding, memoing, sorting and writing the theory. The first stage of living in innocence was originally called living in blissful ignorance. This author's first attempt at sorting had squeezing a lifetime into a moment as the second stage of the theory. However, an additional sort and further reflection allowed emergence of being a good mom/dad in the new 
reality as the second stage with squeezing a lifetime into a moment as a process within that second stage. These modifications are just two made during the grounded theory process; others occurred. Thus, the criterion of modifiability is met.

\section{Limitations}

There are no methodological limitations in this study. Conduct of the study occurred in a manner consistent with classic ground theory methodology. However, there are four characteristics that present as opportunities for further research.

The participants were a relatively homogenous group. All participants were female, except for one male. Additionally, most of the participants currently lived in the same state except for two. Therefore it is important to discover if the grounded theory of living within paradoxes reflects the experience of fathers of babies with life-limiting congenital anomalies and of parents who live in other locales.

The participants were volunteers willing to talk about their painful experience. The theory may just reflect the experience of parents willing to talk about their experience and not those who still cannot talk about their loss. It is unclear how to include those unwilling or unable to share, but their input could potentially modify the theory.

Many of the participants utilized some form of hospice care $(n=7)$. This is not consistent with the literature that suggests that anywhere from 9-20\% of dying infants receive adequate end-of-life care (Feudtner et al, 2002; Friebert \& Huff, 2009; Leuthner, Boldt, \& Kirby 2004; NHPCO, 2001; Zwerdling et al, 2000). In this study, over half of the babies had hospice care. This high percentage reflects the author’s participant recruiting source rather than a genuine increase in access to palliative and end-of-life care for babies. Therefore it would be important 
in another study to recruit more participants who did not have the benefit of palliative or hospice care.

This study only included participants whose babies lived 48 hours to 15 months of age. Many babies who die of life-limiting congenital anomalies only live a few hours after birth. Thus it would be important to explore the experience of parents whose babies lived less than 48 hours to see if that experienced mirrored those who babies lived longer.

\section{Implications for Nursing}

The grounded theory of living within paradoxes is a new theory that has the potential to impact nursing. That impact may be seen in the arenas of education, practice and research. There is a caveat to the application of the theory in these arenas. The nurse must be mindful that this theory describes the experience of a parent who is willing and able to discuss parenting an infant with a life-limiting congenital anomaly. Therefore, the theory may not apply to a parent who is unwilling or unable to discuss their experience. A discussion of the implications for each arena of nursing follows.

\section{Education}

There are significant implications for nursing education that pertain to the theory of living within paradoxes. For the purposes of this discussion, nursing education refers not only to the basic educational preparation of nurses but also the continuing education of practicing nurses. Nursing education at all levels must do a better job of educating nurses, student or practicing, about palliative/end-of-life care in general and specifically about perinatal loss.

Undergraduate students. Students in nursing programs need more exposure to death and dying in the classroom and in clinical settings. An older study by Ferrell, Virani, and Grant (1999) found that only $2 \%$ of nursing textbook content pertained to end-of-life. Given the 
expansion of knowledge in nursing and an already crowded curriculum, it is unlikely that this statistic has changed.

Mallory (2003) opined that nursing education lacks an "effective, efficient approach to educating students about end-of-life care” (Mallory, 2003, p. 306). Therefore a different means is needed. Mallory (2003) demonstrated the effectiveness of the End of Life Nurse Education Consortium (ELNEC) education program along with clinical/lab experiences on improving the attitude of undergraduate nursing students' toward care of the dying. Further discussion of ELNEC occurs later in this section. Smith-Stoner (2009) used simulation to educate nursing students about end-of-life care. Additionally, the simulation experience allowed the student to explore personal ideas and feelings about caring for a dying patient (Smith-Stoner, 2009).

The previous strategies, while effective, only address the general issues of end-of-life care. The specific issue of perinatal loss is not covered. The students in the maternal-child course at the site of this author's employment hear a two hour presentation from a labor and delivery nurse who has special interest in perinatal loss. It is unknown if students in other undergraduate programs across the nation get the same opportunity. There is a separate pediatric palliative care course by ELNEC which includes content related to the death and dying of infants. Baby and child simulators exist. The use of the ELNEC-pediatric palliative course (PPC) and simulators could increase undergraduate students' knowledge of perinatal loss. The theory of living within paradoxes could provide a theoretical framework with which to organize content about perinatal loss in a maternal-child nursing course. Discussion of the living within paradoxes could also occur in discussions of grounded theory in undergraduate nursing research courses. 
Practicing nurses. It is the rare nurse who has not cared for a dying patient. Even those in maternal-child nursing are not exempt from exposure to death and dying. Nurses caring for dying infants and their families must have a working knowledge of the physical and psychospiritual aspects of dying and the theoretical in order to provide appropriate palliative/end-of-life care. Familiarity with the various bereavement, parenting and developmental theories as well as the theory of living within paradoxes is vital.

Practicing nurses are acutely aware of the need for more education about palliative/endof-life care for infants. In a study exploring barriers and facilitators of end-of-life care in the NICU, $100 \%$ of the surveyed nurses agreed with the need for palliative care education but only 46\% reported receiving that education (Wright, Prasun and Hilgenberg, 2011). Caitlin and Carter (2002) stated that an essential element for optimal palliative/end-of-life care for a dying baby and the family was an educated, compassionate, and consistent staff. Therefore, it is vital that orientation for nurses who regularly provide care to the dying infant and their families include competencies related to palliative/end-of-life care. In order to maintain a current knowledge base and skills, palliative/end-of-life care should be included as a competency in the annual competency check-offs required of nurses who care for critically ill and dying infants. The ELNEC-PPC program, simulation and discussion of the theory of living within paradoxes are options to provide basic and refresher education to these nurses. It is important to utilize nurses, who are identified as proficient in delivery of compassionate palliative/end-of-life care, mentor less experienced nurses during the process of caring for a dying infant.

The NICU or PICU is not the only place where dying infants receive nursing care. The data show that most infants die in either a NICU or PICU (Carter et al., 2004; Brandon, Docherty, \& Thorpe, 2007; Feudtner, et al., 2011; Feudtner et al., 2002; Leuthner, Boldt, \& 
Kirby, 2004; Zwerdling et al., 2000). Infants who die in other places such as labor and delivery, post-partum, emergency room, a regular pediatric unit, or home are the recipients of nursing care. Nurses working in out-patient primary care may provide care to an infant with life-limiting congenital anomaly or the expectant mother carrying a fetus with a life-limiting congenital anomaly. Hospice nurses possess basic palliative/end-of-life care knowledge, but may rarely care for dying infants. It is vital that nurses in these other areas learn about perinatal palliative/end-of-life care. Since these nurses have less experience in caring for a dying infant, it is important that a more experienced nurse be available as a mentor so delivery of optimal palliative/end-of-life care occurs.

ELNEC. The American Association of Colleges of Nursing (AACN) and the City of Hope National Medical Center developed the ELNEC program in 2000 to educate nursing students and practicing nurses about palliative care using a train the trainer format. Over 14,000 nurses have attended the train-the-trainer program, those nurses in turn have trained an estimated 400,000 other nurses (AACN, 2012). Additional programs evolved from the original program. Currently there are ELNEC programs for specific patient populations: pediatric, geriatric, critical care, oncology and veterans. There is also a graduate program by which graduate faculty educate advanced practice nursing students about palliative care. The pediatric program provides education about dying children from newborns to adolescents. However this author envisions a program specific for families expecting the birth of a baby with a life-limiting congenital anomaly, an ELNEC-perinatal. This author boldly suggests that the theory of living within paradoxes could provide the theoretical foundation for such a program. 


\section{Practice}

The theory of living within paradoxes has relevance for nurses in many sites of practice with NICU and PICU being the most obvious choices. However, living within paradoxes has application to nurses practicing in primary care offices, regular pediatric unit, labor and delivery, high-risk antepartum and post-partum units, home health, hospice, palliative care, and even emergency room. Advanced practice nurses, such as certified nurse midwives (CNM), neonatal nurse practitioners (NNP), pediatric nurse practitioners (PNP), and family nurse practitioners (FNP) could also utilize living within paradoxes in the primary or acute care setting as a conceptual framework for practice when caring for the pregnant patient carrying a fetus with a life-limiting congenital anomaly or the baby diagnosed with a life-limiting congenital anomaly. Since most infants still die in the NICU, most of the discussion focuses on the implications for practice in the NICU. However, many of the principles guiding care for the dying infant in those units can be extrapolated for use in the other settings in which infants with life-limiting congenital anomalies die.

Protocols/Position Statements/Papers. The theory of living within paradoxes provides evidence for many of the nursing interventions suggested in the papers, position statements, and protocols about palliative/end-of-life care for an infant. For ease of discussion, all of the prescriptive protocols, position statements and general how-to-care for papers will be called protocols. According to Kendall and Guo (2008), most bereavement care that occurs in NICU's has been an "intuitive response to a perceived need" (p.131). This author would argue that this statement is true for many of the palliative/end-of-life protocols. These protocols address issues under the general categories of communication, environment, parental activities, bereavement, withdrawal of life support, pain and symptom control, and prolonged dying process, (Carter \& 
Bhatia, 2001; Catlin \& Carter, 2002; De Lisle-Porter \& Podruchny, 2009; Gale \& Brooks, 2006; NANN, 2010). The first four aspects listed above primarily pertain to the parents and find support in the theory of living within paradox.

Communication. Living within paradoxes provides evidence for the interventions related to communication. Effective communication is vital as parents are getting the bad news and afterwards while those parents are seeking answers and making decisions. Parents need honest and sensitive communication from health care professionals about palliative care while seeking normalcy and during the process of letting go while anticipating the second breaking point of death of the baby. The importance of communication continues through the stage of going on. The nurse who attends the infant's funeral assists the parent in saying goodbye to the baby and communicates to the parent that the baby's life had meaning (seeking meaning in the baby's life). Follow-up phone calls or visits serve as means of getting professional help. During these phone calls the parent should not experience feeling silenced, but rather should be free to tell their story. Thus living within paradoxes supports the significance of communication.

Environment. The NICU environment is one that often forces the parent to experience being separated from the baby when the parent is seeking normalcy and wants to be alone with the baby. Wright et al. (2011) reported that that only 38\% of the surveyed nurses thought the physical environment was ideal for provision of palliative care. Therefore, most of the protocols addressed the need for creation of an optimal environment for the infant's death. Environment refers to both location of care and creation of a milieu.

Some protocols developers suggest offering to move the dying infant out of the NICU to the mother's postpartum room, regular pediatric environment, or even to home is possible (Caitlin \& Carter, 2002; De Lisle-Porter \& Podruchny, 2009; Gale \& Brooks, 2006). Moving the 
baby to the mother's postpartum room meets the parental need of seeking normalcy and being alone with the baby. The last option of taking the baby home mirrors the sub-property of taking the baby home to die. These options all require the parent to participate in making decisions and assume the occurrence of letting go.

Caitlin and Carter (2002) assert that the "location is not as important as the mind-set of the persons involved in end-of-life care” (p. 189). That statement refers to the creation of a milieu appropriate for optimal palliative/end-of-life care. Flexibility of visiting policies, privacy, soft or minimal lighting, minimal noise, a bed for the parents, and provision of snacks are a few of the ways to create a peaceful environment within the NICU and any other unit (Catlin \& Carter, 2002; De Lisle-Porter \& Podruchny, 2009; Gale \& Brooks, 2006). Other ways to achieve an appropriate environment include having an educated, compassionate, and consistent staff to provide care to the dying baby and the family (Caitlin \& Carter, 2002). These milieu interventions may make the parent more comfortable while keeping vigil, assist with seeking normalcy and creating memories during the process of squeezing a lifetime into a moment.

Parenting activities. In the category of parenting activities, one finds significant relevance to living within paradoxes, specifically to the processes and properties in the stage of being a good mom/dad in a new reality. Parenting activities include giving the parents pictures of the infant, taking family photos, touch, kangaroo care, dressing, changing diapers, pumping to provide breast milk, having the baby baptized/dedicated and bathing and dressing the infant (Carter \& Bhatia, 2001; Catlin \& Carter, 2002; De Lisle-Porter \& Podruchny, 2009; Gale \& Brooks, 2006; Gold, 2007; Kavanaugh \& Moro, 2006; Leuthner, 2004; Romesberg, 2007; Toce, 2000; Walden, Sudia-Robinson \& Carrier, 2001). One assumes that parents should be able to touch or hold their baby. However, this does not always happen when an infant's condition is 
critical and unstable. It is often only when letting go occurs that the parent is allowed to hold the baby. There is also support for many of the parent activities in the process of saying goodbye to the baby in the stage of going on.

Some of the activities suggested may seem like insignificant gestures, such as encouraging parents to touch, dress and/or hold their baby. However, these seemingly little gestures have implications for a lifetime of memories. One of the paradoxes discussed in chapter four had to do with the parent not feeling like a mom or dad despite being a good mom/dad in the new reality. NICU nurses play a significant role in the promotion of parental attachment (Hopwood, 2010). Therefore it is incumbent upon the nurse to make sure the parent has the opportunity to make some sort of connection to the baby. The parenting activities are vital to helping the parent feel like a mom or dad and are supported by the theory of living within paradoxes.

Mementos. The creation and giving of mementos to parents expecting and/or experiencing the death of an infant deserves special mention in this discussion. All the protocols discuss the importance of mementos (Carter \& Bhatia, 2001; Catlin \& Carter, 2002; De LislePorter \& Podruchny, 2009; Gale \& Brooks, 2006; Gold, 2007; Kavanaugh \& Moro, 2006; Leuthner, 2004; NANN, 2010; Romesberg, 2007; Toce, 2000; Walden, Sudia-Robinson \& Carrier, 2001). Lisle-Porter and Podruchny (2009) specifically suggested giving the parent a memory box that contains meaningful items and/or items related to the hospitalization. The memory box may contain the expected: a blanket in which the infant was wrapped, hand/footprints, a lock of hair, clothing the baby wore and pictures/disc with pictures. Items related to the hospitalization such as are the infant's blood pressure cuff, a dirty diaper, cord clamp, phototherapy mask or the identification band are the more unexpected items placed in the 
memory box that seem to hold meaning to bereaved parents (De Lisle-Porter \& Podruchny, 2009). Being able to have something tangible that belonged to the baby was important to parents whose baby died of life-limiting anomalies (Brosig et al, 2007). Lambert-Fink (2012) found that women who experienced stillbirth had lower grief scores two years post loss if they received a memory box and had the opportunity to talk about the loss.

Creating memories and keeping mementos occur in two different stages of living within paradoxes. It is during the process of squeezing a lifetime into a moment, while the baby is still alive, that creating memories occurs. One aspect of creating memories is making mementos. Keeping mementos is a property of the process of keeping the baby's memory alive after the baby's death. It is imperative that nurses proactively create opportunities for creating memories and mementos while the baby is still alive. Living within paradoxes provides more evidence of the vital importance of mementos to bereaved parents.

Bereavement care. The last category of care specifically targeted to parents in the protocols is that of bereavement care. This care may start after the cutting point of getting the bad news and during the stage of being a good mom/dad in the new reality since grieving starts after the cutting point of getting the bad news. However, most bereavement care takes place after the cutting point of the death of the baby in the stage of going on. Bereavement care should be congruent with the parents’ preferences in spiritual/religious and cultural domains (Catlin \& Carter, 2002; De Lisle-Porter \& Podruchny, 2009; Gale \& Brooks, 2006). Attendance at a memorial or funeral service by the nurse(s) who cared for the baby is valued by parents (Brosig et al., 2007). Service attendance by the nurse assists the parent in saying goodbye to the baby and seeking meaning in the baby's life. 
Other bereavement care interventions include: follow-up phone calls at specific intervals and on significant days, introducing the parent/family to support groups (online and/or live), and hosting an annual memorial service specific for perinatal loss and inviting the parents (Caitlin \& Carter, 2002; Gale \& Brooks, 2006). These activities serve to assist the parent in saying goodbye to the baby and seeking meaning in the baby's life. The follow-up phone calls and support groups also provide the opportunity for the parent to keep the baby's memory alive by telling their story. As previously discussed, many parents experience feeling silenced when wanting to talk about the deceased baby. The phone call ensures that at least one person will listen. Peers in a support group also will listen and share the common history of the loss of a baby with the parent. The support group also provides the paradoxical opportunities and processes of helping others and receiving help. Therefore, the theory of living within paradoxes provides evidence for many of the bereavement interventions.

Barriers. Many barriers impede optimal end-of-life care for the dying neonate/infant and the family. NICU nurses report inadequate staffing, a non-conducive environment, technological imperatives, parental expectations/demands, moral distress and lack of education as barriers to effective end-of-life care (Kain, 2011; Kohlberg Wright, Prasun, \& Hilgenberg, 2011). All of these issues were addressed by the authors of the various protocols. One need only look at the publication dates of the protocols and the barrier studies to see that things have not changed. More must be done to remove the barriers to ensure that dying infants and their families received optimal end-of-life care.

\section{Research}

There is a great need for more research related to infants with life-limiting congenital anomalies and their families as evidenced by the dearth of published research. Some suggestions 
for additional research were already discussed in the limitations section of this chapter. There are many more possibilities for research. The theory itself may serve as a theoretical or conceptual framework for research in expected perinatal loss.

The stages and specific processes and properties of the theory provide a stepping off point for additional studies. More information is needed about the support and care needed by parents from the first breaking point of getting the bad news through the stage of going on. There is some published research that focuses on the bereavement process that occurs after the cutting point of the death of the baby in the stage of going on. However, as discussed earlier, much of that research lumps all types of perinatal loss together. More research is needed about what helps a parent of baby with a life-limiting congenital anomaly with going on after the expected death of a baby. But researchers also need to explore what is happening between the cutting points of getting the bad news and the death of the baby. There is so much that is unknown about this period of time. Recruitment of participants would be challenging. One could ask if it is right to distract the parent from the process of squeezing a lifetime into a moment in the stage of being a good mom/dad in the new reality to collect data. The information would be invaluable.

The conceptual hypotheses are also a potential source for additional research. One could use qualitative, quantitative or mixed methodologies to test the conceptual hypotheses listed in Chapter 4. This author will provide an example of potential research with the first conceptual hypothesis: the stage of living in innocence ends with the cutting point of getting the bad news. To test this hypothesis using quantitative methods, the researcher would administer the Beck depression inventory to women carrying a fetus with a life-limiting anomaly as well as women carrying a healthy fetus. The researcher would then compare the scores to see if there was a 
greater rate of depression in the mothers who had received bad news and were carrying a fetus likely to die. A phenomenological methodology may be used to explore the lived experience of being pregnant or getting the bad news. Time and space limitation prevent the discussion of each hypothesis and potential research.

The research questions that arise from living within the paradoxes are almost endless. What kind of support or information do parents need after getting the bad news How can nurses or other health care professionals help parents with decision making? What model of care best meets the needs of families facing the inevitable death of their baby? Is perinatal palliative/hospice care an effective model of care? What can NICU staff do to minimize the separation of the baby from the parent and meet the parental need to be alone with the baby? How can nurses assist in the creation of memories and sense of normalcy? How does a parent deal with the day-to-day when there is an uncertain amount of time with the baby? What supports and services need to be in place so parents can take their dying babies home? Many more researchable questions exist.

Additionally, research is needed to further develop the stages, processes, and properties of the theory. Further development of the theory would allow nurses and other health care practitioners to explain and predict the experience of parents of infants with life-limiting congenital anomalies. Greater understanding of the experience will allow for development and testing of interventions based on evidence.

Finally, one could expand the focus of investigation to individuals of all ages who are suffering from life-limiting illnesses. This author sees many correlates with the stages and processes of this theory and personal experience as a hospice nurse. Since paradox is a universal 
human experience, it is conceivable a formal theory of living within paradoxes could evolve from this grounded theory.

\section{Conclusions}

The theory of living within paradoxes is a new theory that describes the process of parenting an infant with a life-limiting congenital anomaly. There is some congruence with the findings of the scant research conducted and stages, processes, properties and sub-properties of living within paradoxes. One also finds relevance with this new theory and the existing theoretical literature in death and dying, bereavement/grief, parenting, and paradox. Emergence of living with paradoxes arose from the life experiences of participants whose baby died from a life-limiting congenital anomaly, rather than this author's preconceived notions. Given that emergence, living within paradoxes meets the criteria of fit, work, reliability and modifiability. This new theory has significance implications for nursing education, practice, and research.

There has been a significant increase in interest about infant palliative/end-of-life care in the last decade. The literature related to this topic is still mostly in the form of protocols and position papers, although there is more research devoted to this topic. Most of the research focuses on topics such as barriers to palliative/end-of-life care in the NICU, withdrawal of life support, and bereaved parents. There are even a few studies research exploring the experience of parents whose baby dies from life-limiting congenital anomalies. Despite the increased interest and research, there still exists a significant knowledge gap. The grounded theory of Parents Living within the Paradoxes of Infant Death from Life-Limiting Anomaly fills a unique and needed niche in the literature. Living within paradoxes describes the process of parents throughout the entirety of the experience, from healthy pregnancy to life after the death of the baby. In contrast, most of the research only explores the bereavement experience of the parent 
after the death of the infant. It is important for health care professionals to understand the entirety of the parent's experience in order to develop and test appropriate interventions to help at all points of the process, not just at and after death. The theory of living within paradoxes provides a conceptual framework for the development and testing of those interventions. There is much to do to ensure that babies dying from life-limiting congenital anomalies have optimal palliative/end-of-life care to ensure a good death. It is this author's hope that Parents Living within the Paradoxes of Infant Death from Life-Limiting Anomaly will serve as a starting place. 


\section{References}

Abe, N., Caitlin, A, \& Mihara, D. (2001). End of life in the NICU: A study of ventilator withdrawal. American Journal of Maternal-Child Nursing, 26(3), 141-146. Retrieved from http://journals.lww.com/mcnjournal/pages/default.aspx

Adams, D., Corr, C., Davies, B., \& Deveau, E. (1999). Children, adolescents, and death: Myths, realities, and challenges. Death Studies, 23(5), 443-463. doi:10.1080/074811899200957

Andressen, E. \& Toce, S. (2002). Research on the quality of death of children. Quality of life Newsletter 28:17-18, 2002. Retrieved from http://www.cardinalglennon.com

American Academy of Pediatrics Committee on Bioethics and Committee on Hospital Care (2000). Palliative care for children. Pediatrics, 106, 351-357. Retrieved from http://pediatrics.aappublications.org/content/106/2/351.full

Annells, M. (1996). Grounded theory method: Philosophical perspectives, paradigm of inquiry and postmodernism. Qualitative Health Research, 6(3), 379-393. doi:10.1177/104973239600600306

Annells, M. (1997). Grounded theory method, part I: Within the five moments of qualitative research. Nursing Inquiry, 4, 120-129. doi:10.1111/j.1440-1800.1997.tb00085.x

Arnold, J. \& Gemma, P. (2008). The continuing process of parental grief. Death Studies, 32(7), 658-673. doi:10.1080/07481180802215718

Barr, P. \& Cacciatore, J. (2008). Personal fear of death and grief in bereaved mothers. Death Studies, 32(5), 445-460. doi:10.1080/07481180801974752

Barrerra, M., D’Agostino, N., Schneiderman, G., Tallett, S., Spencer,L., \& Haovcevska, V. (2007). Patterns of parental bereavement following the loss of a child and related factors. Omega: Journal of Death \& Dying, 55(2), 145-167. doi:10.2190/OM.55.2.d 
Barrera, M., O’Connor, K. D’Agostino, N., Spencer, L., Jovcevska, V., et al. (2009). Early parental adjustment and bereavement after childhood cancer death. Death Studies, 33(6), 497-520. doi:10.1080/07481180902961153

Benoliel, J. (1996). Grounded theory and nursing knowledge. Qualitative Health Research, 6(3), 4106-428. doi:10.1177/104973239600600308

Belasco, J., Danz, P., Drill, A., Schmid, W., \& Burkey, E. (2000). Supportive care: Palliative care in children, adolescents, and young adults - models of care, interventions and cost of care: A retrospective review. Journal of Palliative Care, 16(4), 39. Retrieved from http://www.criugm.qc.ca/journalofpalliativecare/index.html

Bhatia, J. (2006). Palliative care in the fetus and newborn. Journal of Perinatology, 26, s24-s26. doi:10.1038/sj/jp7211468

Black, D. (1998). Bereavement in childhood. British Medical Journal, 316 (7151), 931 doi: 10.1136/bmj.316.7135.931

Black, B. \& Sandelowski, M. (2010). Personal growth after severe fetal diagnosis. Western Journal of Nursing Research, 32(8), 1011-1030. doi:10.1177/0193945910371215

Blumer, H. (1969). Symbolic interaction: Perspective and method. Englewood Cliffs, NJ: Prentice Hall.

Brackley, K., Kilby, M., Wright, J., \& Brawn, W. (2000). Outcome after prenatal diagnosis of hypoplastic left-heart syndrome: A case series. The Lancet, 1143(9236), 356. doi:10.1016/S0140-6736(00)02756-2

Branchett, K. \& Stretton, J. (2012). Neonatal palliative and end-of-life care: What parents want from professionals. Journal of Neonatal Nursing, 18, 40-44. Retrieved from www.elsevier.com/jneo 
Brandon, D., Docherty, S., \& Thorpe, J. (2007). Infant and child deaths in acute care settings: Implications for palliative care. Journal of Palliative Medicine, 10(4), 910-918. doi: 10.1089/jpm.2006.0236

Breeze, A., Lees, C., Kumar, A., Missfelder-Lobos, H., \& Murdoch, E. (2007). Palliative care for prenatally diagnosed lethal fetal abnormality. Archives of Diseases in Childhood: Fetal and Neonatal Edition, 92(1), F56-F58. doi: 10.1136?adc.2005.092122

Brosig, C., Pierucci, R., Kupst, M. \& Leuthner, S. (2007). Infant end-of-life care: The parents’ perspective. Journal of Perinatology, 27, 510-516. doi:10.1038/sj.jp7211755

Bryant, A. \& Charmaz, K. (2007). Grounded theory in historical perspective: An epistemological account. In A. Bryant \& K. Charmaz (Eds). The Sage Handbook of Grounded Theory (pp.31-57). Thousand Oaks, California: Sage.

Buglass, E. (2010). Grief and bereavement theories. Nursing Standard, 24(41), 44-47. Retrieved from nursingstandard.rcnpublishing.co.uk/

Caitlin, A. \& Carter, B. (2002). State of the art. Creation of a neonatal end-of-life palliative care protocol. Journal of Perinatology, 22(3),184-195. doi: 10.1038/sj/jp/7210687

Carter, B. (2007). Neonatal and infant death: What bereaved parents can teach us. Journal of Perinatology, 27(8), 467-468. doi:10.1038/sj.jp.7211777

Carter, B. S. \& Bhatia, J. (2001). Comfort/palliative care guidelines for neonatal practice: Development and implementation in an academic medical center. Journal of Perinatology, 21(5), 279-283. Retrieved from http://www.nature.com/jp/journal/v21/n5/pdf/7210582a.pdf 
Carter, B., Howenstein, M., Gilmer, M., Throop, P., France, D., \& Whitlock, J. (2004). Circumstances surrounding the deaths of hospitalized children: Opportunities for pediatric palliative care. Pediatrics, 114 (3), e361-e366. doi: 10.1542/peds.2003-0654-F

Cavaliere, T. (2007). Should neonatal palliative care take place at home rather than the hospital? Maternal-Child Nursing Journal, 32(5), 270.

doi:10.1097/01.NMC.0000287994.13179.43

Center for Disease Control (1999). Achievements in public health, 1990-1999: Healthier mothers and babies. Morbidity and Mortality Weekly Report, 48(38), 849-858. Retrieved from http://www.cdc.gov/mmwr/preview/mmwrhtml/mm4838a 2.htm.

Chandra, S. \& Spitzer, A. R. (2001). Congenital diaphragmatic hernia: Prenatal diagnosis, outcome and continuing morbidity in survivors. Clinical Pediatrics, 40(1), 57-58. doi: 10.1177/000992280104000110

Charmaz, K.(2000). Grounded theory: Objectivist and constructivist methods. In N.K. Denzin \& Y. S. Lincoln (Eds.). Handbook of Qualitative Research (2nd ed., pp. 509-535). Thousand Oaks, California: Sage.

Children's Hospice International (n.d.). Who we are. Retrieved from http://www.chionline.org/resources/about.phtml

Chivotti, R. \& Piran, N. (2003). Rigour and grounded theory research. Journal of Advanced Nursing, 44(4), 427-435. doi:10.1046/j.0309-2402.2003.02822.x

Cohen, L. M., Poppel, D. M., Cohn, G. M., \& Reiter, G. S. (2001). A very good death: Measuring quality of dying in end-stage renal disease. Journal of Palliative Medicine, 4(2),167-172. doi:10.1089/109662101750290209. 
Copp, G. (1998). A review of current theories of death and dying. Journal of Advanced Nursing, 28(2), 382-390. doi: 10.1046/j.1365-2648.1998.00794.x

Costello, J. \& Trinder-Brook, A. (2000). Children's nurses' experiences of caring for children in hospital. Paediatric Nursing, 12(6), 28-31. Retrieved from http://nursingchildrenandyoungpeople.rcnpublishing.co.uk/

Craig, F. \& Goldman, A. (2003). Home management of the dying NICU patient. Seminars in Neonatology, 8(2), 177-183. doi:10.1016/S1084-2756(02)00223-3

Curtis, J., Patrick, D., Engelberg, R., Norris, K., Asp, C., \& Byock, I. (2002). A measure of the quality of dying and death. Initial validation using after-death interviews with family members. Journal of Pain and Symptom Management, 24(1), 17-31. Retrieved from http://www.journals.elsevier.com/journal-of-pain-and-symptom-management/

Cutcliffe, J. (2000). Methodological issues in grounded theory. Journal of Advanced Nursing, 31(6), 1476-1484. doi:10.1046/j.1365-2648.2000.01430.x

Dastgiri, S., Gilmour, W. H., \& Stone, D. H. (2003). Survival of children born with congenital anomalies. Archives of Disease in Childhood, 88(5), 391-394. doi:10.1136/adc.88.5.391

DeCinque, N., Monterosso, L., Dadd, G., Sidhu, R., Macpherson, R., \& Aoun, S. (2006). Bereavement support for families following the death of a child from cancer: Experience of bereaved parents. Journal of Psychosocial Oncology, 24(2), 65-83. doi:10.1300/J077v24n02-05

De Lisle-Porter, M. \& Podruchny, A. (2009). The dying neonate: Family-centered end-of-life care. Neonatal Network, 28(2), 75-83. doi: 10.1891/0730-0832.28.2.75

DeSpelder, L. \& Strickland, A. (2002). The last dance: Encountering death and dying. Boston, MA: McGraw Hill. 
Downey, V., Bengiamin, M., Heuer, L., \& Juhl, N. (1995). Dying babies and associated stress in NICU nurses. Neonatal Network: The Journal of Neonatal Nursing, 14, 41-46. Retrieved from http://neonatalnetwork.metapress.com/content/121252

Duff, R. \& Campbell, A. (1973). Moral and ethical dilemmas in the special-care nursery. New England Journal of Medicine, 289(17), 890-894. Retrieved from http://www.nejm.org/

Ellison, S. \& Fuller, J. D. (1998). A good death? Finding a balance between the interests of patients and caregivers. Generations, 22, 87-91. Retrieved from http://www.asaging.org/publications

Emanuel, E. J. \& Emanuel, L. L. (1998). The promise of a good death. Lancet, 351, SII21. doi:10.1016/S0140-6736(98)90329-4

Engelder, S., Davies, K., Zeilinger, T., \& Rutledge, D. (2012). A model program for perinatal palliative services. Advances in Neonatal Care, 2(1), 28-36.

doi: 10.1097/ANC.0b013e318244031c

Eronen, M. (1997). Outcome of fetuses with heart disease diagnosed in utero. Archives of Disease in Childhood, Fetal and Neonatal Edition, 77(1), F41-46. doi:10.1136/fn.77.1.F41

Feeg, V. (2005). Challenging the assumptions for better pediatric palliative care. Pediatric Nursing, 31(2), 77\& 86. Retrieved from http://www.pediatricnursing.net/

Ferrell, B., Virani, R. \& Grant, M. (1999). Analysis of end-of-life content in nursing textbooks. Oncology Nursing Forum, 26(5), 869-876. Retrieved from http://www.ons.org/Publications/ONF/ 
Feudtner, C., Christakis, D. A., Zimmerman, F. J., Muldoon, J. H., Neff, J. M., \& Koepsell, T. D. (2002). Characteristics of deaths occurring in children's hospitals: Implications for supportive care services. Pediatrics, 109(5), 887-893. doi: 10.1542/peds.109.5.887

Feudtner, C., DiGiuseppe, D., \& Neff, J. (2003). Hospital care for children and young adults in the last year of life: A population-based study. BMC Medicine, 1(1), 1-13. doi:10.1186/1741-7015-1-3

Feudtner, C., Kang, T., Hexem, K., Friedrichsdorf, S., Osenga, K., Siden, H.,......Wolfe, J. (2011). Pediatric palliative care patients: A prospective multicenter cohort study. Pediatrics, 127(6), 1094-1101. doi: 10.1542/peds.2010-3225

Feudtner, C., Silveira, M., \& Christakis, D. A. (2002). Where do children with complex chronic conditions die? Patterns in Washington state, 1980-1998. Pediatrics, 109(6), 656-660. doi: 10.1542/peds.109.4.656

Feudtner, C. (2004). Perspectives on quality at the end of life. Archives of Pediatrics \& Adolescent Medicine, 158(5), 415. Retrieved from http://archpedi.jamanetwork.com/journal.aspx

Field, M. \& Behrman, R. (2003). When children die: Improving palliative and end-of-life care for children and their families. Washington, D.C.: National Academies Press.

Field, M. \& Cassel, C. E. (1997). Approaching death: Improving care at the end of life (summary). Retrieved from http://books.nap.edu/catalog/5801.html

Forbes, M. A. \& Rosdahl, D. R. (2003). The final journey of life. Journal of Hospice and Palliative Nursing, 5(4). 213-20. Retrieved from http://journals.lww.com/jhpn/pages/default.aspx 
Friebert, S. (2009). NHPCO facts and figures: Pediatric palliative and hospice care in America. Retrieved from NHPCO website: http://www.nhpco.org/files/public/quality/Pediatric_Facts-Figures.pdf

Friebert, S. \& Huff, S. (2009). NHPCO’s pediatric standards: A key step in advancing care for America’s children. NewsLine. Retrieved from NHPCO website: http://www.nhpco.org/files/public/quality/Peds-Standards_article_NL-0209.pdf

Gale. G., \& Brooks, A. (2006). Implementing a palliative care program in a newborn intensive care unit. Advances in Neonatal Care, 6(1), 37-53. doi: 10.1016/j.adnc.2005.11.004

Gazelle, G. (2003). A good death: not just an abstract concept. Journal of Clinical Oncology, 21, 95S-96S. doi:10.1200/JCO.2003.01.185.

Gold, K. (2007). Navigating care after a baby dies: A systematic review of parent experiences with health providers. Journal of Perinatology, 27(4), 230-237. doi:10.1038/sj.jp.7211676

Glaser, B. (1978). Theoretical sensitivity: Advances in the methodology of grounded theory. Mill Valley, California: The Sociology Press.

Glaser, B. (1998). Doing grounded theory: Issues and discussions. Mill Valley, California: Sociology Press

Glaser, B. (2006). The roots of grounded theory. The Grounded Theory Review, 5(2/3). Retrieved from http://groundedtheoryreview.com/

Glaser, B. \& Strauss, A. (1965). Awareness of dying. Mill Valley, CA: Sociology Press.

Glaser, B. \& Strauss, A. (1968). Time for dying. Mill Valley, CA: Sociology Press.

Hart, B., Sainsbury, P., \& Short, S. (1998). Whose dying? A sociological critique of the 'good death'. Mortality, 3, 65-77. doi:10.1080/713685884 
Heath, H. \& Cowley, S. (2004). Developing a grounded theory approach: A comparison of Glaser and Strauss. International Journal of Nursing Studies, 41, 141-150. doi:10.1016/S0020-7489(03)00113-5

Himelstein, B. (2006). Pediatric care for infants, children, adolescents and their families. Journal of Palliative Medicine, 9, 163-181. doi:10.1089/jpm.2006.9.163

Hodde, N. M., Engelberg, R. A., Treece, P. D., Steinberg, K. P., \& Curtis, J. R. (2004). Factors associated with nurse assessment of the quality of dying and death in the intensive care unit. Critical Care Medicine, 32, 1648-1653.

doi:10.1097/01.CCM.0000133018.60866.5F

Hopkinson, J. \& Hallett, C. (2002). Good death? An exploration of newly qualified nurses' understanding of good death. International Journal of Palliative Nursing, 8, 532-539. Retrieved from http://www.journals.elsevier.com/international-journal-of-nursingstudies/

Hopwood, R. (2010). The role of the neonatal nurse in promoting parental attachment in the NICU. Infant, 6(2), 54-58. Retrieved from http://www.infantgrapevine.co.uk/

Hutton, N. (2002). Pediatric palliative care: The time has come. Archives of Pediatrics \& Adolescent Medicine, 156(1), 9. Retrieved from http://archpedi.jamanetwork.com/journal.aspx

Initiative for Pediatric Palliative Care [IPPC] (2003). About IPPC. Retrieved from http://www.ippcweb.org/print/printabout.asp

Jeon, Y. (2004). The application of grounded theory and symbolic interactionism. Scandinavian Journal of Caring Sciences, 18(3), 249-256. doi:10.1111/j.1471-6712.2004.00287.x 
Kain, V . (2011). Exploring the barriers to palliative care practice in neonatal nursing: A focus group study. Neonatal, Paediatric and Child Health Nursing, 14(1), 9-14. Retrieved from www.npchn.com/

Kavanaugh, K \& Moro, T. (2006). Supporting parents after stillbirth or newborn death. American Journal of Nursing, 106(9), 74-79. Retrieved from http://journals.lww.com/ajnonline/pages/default.aspx

Kazak, A. \& Noll, R. (2004). Child death from pediatric illness: Conceptualizing intervention from a family systems and public health perspective. Professional Psychology: Research and Practice, 35, 219-226. doi: 10.1037/0735-7028.35.3.219

Keesee, N., Currier, J., \& Neimeyer, R. (2008). Predictors of grief following the death of one's child: The contribution of finding meaning. Journal of Clinical Psychology, 64(10), 1145-1163. doi:10.1002/jclp.20502

Kim, S. \& Lee, Y. (2003). Korean nurses' attitudes to good and bad death, life-sustaining treatment and advance directives. Nursing Ethics, 10(6), 624-637. doi:10.1191/0969733003ne652oa

Kochanek, K., Xu, J., Murphy, S. , Miniño, A., \& Kung, H-S. (2011). National vital statistics reports. Deaths: Final data for 2009 (Vol. 60, \#3). Retrieved from Center for Disease Control website: http://www.cdc.gov/nchs/data/nvsr/nvsr60/nvsr60_03.pdf

Kubler-Ross, E. (1969). On death and dying. New York, New York: Simon \& Schuster.

Lambert Fink, P. (2012). The relationships between perinatal loss interventions at the time of stillbirth and maternal grief. (Unpublished doctoral dissertation). Retrieved from West Virginia University, Morgantown, West Virginia. 
Last Acts. (2002). Care must improve from children with chronic and terminal conditions.

Retrieved from http://www.lastacts.org/statsite/37611a-mrc-newsrelease.html

Lathrop, A. \& VandeVusse, L. (2011). Continuity and change in mothers' narrative of perinatal hospice. Journal of Perinatal \& Neonatal Nursing, 25(1), 21-31.

doi: 10.1097/JPN.0b013e3181fa9c60

Leuthner, S. (2004). Palliative care of the infant with lethal anomalies. Pediatric Clinics of North America, 51(3), 747-759. doi:10.1016/j.pcl.2004.01.006

Leuthner, S., Boldt, A., \& Kirby, R. (2004). Where infants die: Examination of place of death and hospice/home health care options in the state of Wisconsin. Journal of Palliative Medicine, 7(2), 269-277. doi:10.1089/109662104773709396

Leuthner, S. \& Lanberg Jones, E. (2007). Fetal concerns program: A model for perinatal palliative care. Maternal Child Nursing Journal, 32(5), 272-278.

doi: 10.1097/01.NMC.0000287996.90307.c6

Leuthner, S. \& Pierucci, R. (2001). Experience with neonatal palliative care consultation at the Medical College of Wisconsin Children's Hospital of Wisconsin. Journal of Palliative Medicine, 4(1), 39-47. doi:10.1089/109662101300051960

Levy, C., Ely, E., Payne, K., Engelberg, R., Patrick, D., \& Curtis, J. (2005). Quality of dying and death in two medical ICUs. Chest, 127(5), 1775. doi: 10.1378/chest.127.5.1775

Li, J., Hansen Precht, D., Mortensen, P. B., \& Olsen, J. (2003). Mortality in parents after death of a child in Denmark: A nationwide follow-up study. The Lancet, 361(9355), 363- 367. doi:10.1016/S0140-6736(03)12387-2

Lincoln, Y \& Guba, E. (1985). Naturalistic inquiry. Beverly Hills, CA: Sage. 
Lindemann, E. (1944). Symptomatology and management of acute grief. The American Journal of Psychiatry, 151 (6 supp.), 155-160. Retrieved from ajp.psychiatryonline.org/

Lobo, M. (2005). Descriptive research is the bench science of nursing. Western Journal of Nursing Research, 27(1), 5-8. doi:10.1177/0193945904271298

Mak, J. M. H. \& Clinton, M. (1999). Promoting a good death: An agenda for outcomes research a review of the literature. Nursing Ethics, 6, 97-106. doi:10.1177/096973309900600202

Mallory, J. (2003). The impact of a palliative care educational component on attitudes toward care of the dying in undergraduate nursing students. Journal of Professional Nursing, 19 (5), 305-312. doi:10.1016/S8755-7223(03)00094-2

Mangurten, H.H. (1990). Home death and hospital follow-up of the dying infant. American Journal of Perinatology, 7(4), 302-306. Retrieved from http://www.nature.com/jp/index.html

Marcellus, L. (2005). The grounded theory method and maternal-infant research practice. Journal of Obstetric, Gynecologic, and Neonatal Nursing, 34(3), 349-357. doi: $10.1177 / 0884217505276053$

Mathews, T., MacDorman, M., \& Menacker, F, Mathews, T., MacDorman, M., \& Menacker, F. (2002). Infant mortality statistics from the 1999 period linked birth/infant death data set. Retrieved from www.cdc.gov/nchs/data/nvsr/nsvr50-04.pdf

McCann, T. \& Clark, E. (2003). Grounded theory in nursing research: Part 1-Methodology. Nurse Researcher, 11(2), 7-11. Retrieved from http://nurseresearcher.rcnpublishing.co.uk/ 
Meert, K., Briller, S., Schim, S., Thurston, C., \& Kabel, A. (2009). Examining the needs of bereaved parents in the pediatric intensive care unit: A qualitative study. Death Studies, 33(8), 712-740. doi:10.1080/07481180903070434

Mercer, R. (2004). Becoming a mother versus maternal role attainment. Journal of Nursing Scholarship, 36(3), 226-232. doi:10.1111/j.1547-5069.2004.04042

Milo, E. M. (1997). Maternal responses to the life and death of a child with a developmental disability: A story of hope. Death Studies, 21(5), 443-476. doi:10.1080/074811897201822

Milstein, J. M. (2003). Detoxifying death in the neonate:In search of meaningfulness at the end of life. Journal of Perinatology, 23(4), 333-336. doi:10.1038/sj.jp.7210903

Montgomery, P. \& Bailey, P. (2007). Field notes and theoretical memos in grounded theory. Western Journal of Nursing Research, 9(1), 65-79. doi:10.1177/0193945906292557

Moro, T., Kavanaugh, K., Okuno-Jone, S., VanKleel, J. (2006). Neonatal end-of-life care: A review of the research literature.. Journal of Perinatal \& Neonatal Nursing, 20(3), 262273. Retrieved from http://journals.lww.com/jpnnjournal/pages/default.aspx

Morse, J., Barrett, M., Mayan, M., Olson, K \& Spiers, J. (2002). Verification strategies for establishing reliability and validity in qualitative research. International Journal of Qualitative Methods, 1(2), 1-19. Retrieved from http://ejournals.library.ualberta.ca/index.php/IJQM/article/view/4603/3756

Mularski, R., Curtis, J. R., Osborne, M., Engelberg, R. A., \& Ganzini, L. (2004). Agreement among family members in their assessment of the quality of dying and death. Journal of Pain and Symptom Management, 28, 306-315. doi:10.1016/j.jpainsymman.2004.01.008 
Mularski, R. A., Heine, C. E., Osborne, M. L., Ganzini, L., \& Curtis, J. R. (2005). Quality of dying in the ICU:Ratings by family members. Chest, 128(1), 280-287 doi:10.1378/chest.128.1.280

Nathaniel, A. (2009). An integrated philosophical framework that fits grounded theory. Unpublished manuscript.

National Association of Neonatal Nurses. (2010). Palliative care for neonates and infants: Position statement \#3051. Retrieved from http://www.nann.org/uploads/files/Palliative_Care-final2-in_new_template_01-07-11.pdf

National Perinatal Association. (2009). Palliative care position paper. Retrieved from http://www.nationalperinatal.org/advocacy/pdf/Palliative-Care.pdf

National Center for Health Statistics. (2009). Fast stats: Infant health. Retrieved from http://www.cdc.gov/nchs/fastats/infant_health.htm.

National Consensus Project for Quality Palliative Care. (2009). Clinical practice guidelines for quality palliative care, $2^{\text {nd }} \mathrm{ed}$. Retrieved from http://www.nationalconsensusproject.org National Hospice and Palliative Care Organization. (2001). A call for change: Recommendations to improve the care of children living with life-threatening conditions. Retrieved from www.nhpco.org/files/public/ChIPPSCallforChange.pdf

Papadatou, D., Bellali, T., Papazoglou, I., \& Petraki, D. (2002). Greek nurse and physician grief as a result of caring for children dying of cancer. Pediatric Nursing, 28(4), 345-350. Retrieved from http://www.pediatricnursing.net/

Paradox. (1993). In P. B. Gove (Ed.), Webster's third international dictionary of the English language. Springfield, MA: Merriam Webster, Inc. 
Parkes, C. (1998). Bereavement in adult life. British Medical Journal, 316 (7134), 1-9. doi: 10.1136/bmj.316.7134.856

Parse, R.R. (2007). The humanbecoming school of thought in 2050. Nursing Science Quarterly, 20(4), 308-311. doi: 10.1177/0894318407307160

Patrick, D. L., Curtis, J. R., Engelberg, R. A., Nielsen, E., \& McCown, E. (2003). Measuring and improving the quality of dying and death. Annals of Internal Medicine, 139(5), 410-416. Retrieved from http://annals.org/journal.aspx

Pierson, C. M., Curtis, J. R., \& Patrick, D. L. (2002). A good death: A qualitative study of patients with advanced AIDS. AIDS Care: Psychological \& Socio-Medical Aspects of AIDS/HIV, 14, 587-598. doi:10.1080/0954012021000005416

Pierucci, R. L., Kirby, R. S., \& Leuthner, S. R. (2001). End-of-life care for neonates and infants: The experience and effects of a palliative care consultation service. Pediatrics, 108(3), 653-660. doi: 10.1542/peds.108.3.653

Plato (2009). The Socratic dialogues. (B. Jowett, Trans.). New York, New York: Kaplan Polit, D. \& Beck, C. (2004). Nursing research: Principles and methods. Philadelphia. PA: Lippincott, Williams \& Wilkins.

Rachael, B. (2006). Grieving the death of a child. British Medical Journal, 332(7542), 620-621. doi: $10.1136 / \mathrm{bmj} .332 .7542 .620$

Rempel, G. \& Harrison, M. (2007). Safeguarding precarious survival: Parenting children who have life-threatening heart disease. Qualitative Health Research, 17(6), 824-837. doi:10.1177/1049732307303164

Roberts, P., Priest, H.\& Traynor, M. (2006). Reliability and validity in research. Nursing Standard, 20, 41-45. Retrieved from http://nursingstandard.rcnpublishing.co.uk/ 
Robison, N. J., Hodgman, J. E., Barton, L., \& Pavlova, Z. (2003). Causes of nursery death beyond the neonatal period. Journal of Perinatology, 23, 142-147. doi:10.1038/sj.jp.7210863

Rogers, C., Floyd, F., Seltzer, M., Greenberg, J., \& Jinkuk, H. (2008). Long-term effects of the death of a child in parents' adjustment in midlife. Journal of Family Psychology, 22(2), 203-211. doi:10.1037/0893-3200.22.2.203

Rolfe, G. (2006). Validity, trustworthiness and rigour: Quality and the idea of qualitative research. Journal of Advanced Nursing, 53(3), 304-310. doi: 10.1111/j.13652648.2006.03727.x

Romesberg, T. (2004). Understanding grief: A component of neonatal palliative care. Journal of Hospice and Palliative Nursing, 6(3), 161-170. Retrieved from http://journals.lww.com/jhpn/pages/default.aspx

Rushton, C. H. \& Catlin, A. (2002). Pediatric palliative care: The time is now! Pediatric Nursing, 28, 57. Retrieved from http://www.pediatricnursing.net/

Ryan-Nicholls, K. \& Will, C. (2009). Rigour in qualitative research: Mechanisms for control. Nurse Researcher, 16(3), 70-77. Retrieved from http://nurseresearcher.rcnpublishing.co.uk/

Sandler, I., Kennedy, C., \& Shapiro, E. (2004). Parental grief and palliative care require attention. Archives of Pediatrics \& Adolescent Medicine, 158(6), 590. Retrieved from http://archpedi.jamanetwork.com/journal.aspx

Salomon, L., Belouet, C., Vinant-Binam, P., Sicard, D., \& Vidal-TrӨcan, G. (2001). A terminal care support team in a Paris university hospital: Care providers' views. Journal of Palliative Care, 17(2), 109-16. Retrieved from http://www.liebertpub.com/jpm 
Scott, E. S. (2002). Fetal, perinatal, and infant death with congenital renal anomaly. Archives of Disease in Childhood, 87(2),114. doi:10.1136/adc.87.2.114

Smith-Stoner, M. (2009). Using high-fidelity simulation to educate nursing students about endof-life care. Nursing Education Perspectives, 30 (2), 115-120. Retrieved from http://www.nln.org/nlnjournal/index.htm

Steinhauser, K. E., Bosworth, H. B., Clipp, E. C., McNeilly, M., Christakis, N. A., Parker, J. , Tulsky, J. (2002). Initial assessment of a new instrument to measure quality of life at the end of life. Journal of Palliative Medicine, 5(6), 829-841. doi:10.1089/10966210260499014

Steinhauser, K. E., Clipp, E. C., McNeilly, M., Christakis, N. A., McIntyre, L. M., \& Tulsky, J. A. (2000). erspective. In search of a good death: Observations of patients, families, and providers. Annals of Internal Medicine, 132, 825-832. Retrieved from http://annals.org/article.aspx?articleid=713475

Stephenson, J. 2000). alliative and hospice care needed for children with life-threatening conditions. Journal of the American Medical Association, 284(4), 2437-2438. doi:10.1001/jama.284.19.2437

Stroebe, M. \& Schut, H. (2010). The dual process model of coping with bereavement: A decade on. Omega, 61(4), 273-289. doi:10.2190/OM.61.4.b

Sumner, L., Kavanaugh, K., \& Moro, T. (2006). Extending palliative care into pregnancy and the immediate newborn period: State of the practice of perinatal palliative care. Journal of Perinatal and Neonatal Nursing, 20(1), 113-116. Retrieved from http://journals.lww.com/jpnnjournal/pages/default.aspx 
Tan, J., Docherty, S., Barfield, R., \& Brandon, D. (2012). Addressing parental bereavement support needs at the end of life for infants with complex chronic conditions. Journal of Palliative Medicine, 15(5), 579-584. doi:10.1089/jpm.2011.0357

Tan, G., Totapally, B., Torbati, D., \& Wolfsdorf, J. (2006). End of life decisions and palliative care in a children's hospital. Journal of Palliative Medicine, 9(2), 332-342. doi:10.1089/jpm.2006.9.332

Ternestedt, B., Andershed, B., Eriksson, M., \& Johansson, I. (2002). A good death: Development of a nursing model of care. Journal of Hospice and Palliative Nursing, 4, 153-160. Retrieved from http://journals.lww.com/jhpn/pages/default.aspx

Toce, S. (2000). Applying hospice principles to the care of the high-risk newborn. In Children's International Project on Palliative/Hospice Services (CHIPPS), Compendium of Pediatric Palliative Care, pp. 14-21.Alexandria, VA.: National Hospice and Palliative Care Organization.

Toller, P. (2008). Bereaved parents' negotiation of identity following the death of a child. Communication Studies, 59(4), 306-321. doi:10.1080/10510970802467379

Tuls Halstead, M. \& Hull, M. (2001). Struggling with paradoxes: The process of spiritual development in women with cancer. Oncology Nursing Forum, 28(10), 1534-1544. Retrieved from http://www.ons.org/Publications/ONF/

Vig, E. K., Davenport, N. A., \& Pearlman, R. A. (2002). Good deaths, bad deaths, and preferences for the end of life: A qualitative study of geriatric outpatients. Journal of the American Geriatrics Society, 50(9), 1541-1548. doi: 10.1046/j.1532-5415.2002.50410.x

Vig, E. K. \& Pearlman, R. A. (2004). Good and bad dying from the perspective of terminally ill men. Archives of Internal Medicine, 164(9), 977-981. doi:10.1001/archinte.164.9.977 
Walden, M., Sudia-Robinson, T., \& Carrier,C. (2001). Comfort care for infants in the neonatal intensive care unit at end of life. Newborn and Infant Nursing Reviews, 1(2), 97-105. doi:10.1053/nbin.2001.25436

Wall, S. \& Partridge, J. (1997). Death in the intensive care nursery: Physician practice of withdrawing and withholding life support. Pediatrics, 99(1), 64-70. doi: 10.1542/peds.99.1.64

Walters, G. (2004). Is there such a thing as a good death? Palliative Medicine, 18, 404-408. doi: 10.1191/0269216304pm908oa

Welch, S.(2008). Can the death of a child be good? Journal of Pediatric Nursing, 23(2), 120125. doi:10.1016/j.pedn.2007.08.015

Whitfield, J., Siegel, R., Glicken, A., Harmon, R., Powers, L., \& Goldson, E. (1982). The application of hospice concepts to neonatal care. American Journal of Diseases of Children, 136, 421-424. Retrieved from http://archpedi.jamanetwork.com/issues.aspx

Widger, K. \& Picot, C. (2008). Parents’ perceptions of the quality of pediatric and perinatal endof-life care. Pediatric Nursing, 34(1), 53-58. Retrieved from www.pediatricnursing.net

Williams, C., Cairnie, J., Fines, V., Patey, C., Schwarzer, K., ...\& Kirpalani, H. (2009). Construction of a parent-derived questionnaire to measure end-of-life care after withdrawal of life-sustaining treatment in the neonatal intensive care unit. Pediatrics, 123(1), e87-e95. doi:10.1542/peds.2007-2950

Wright, V., Prasun, M., \& Hilgenberg, C. (2011). Why is end-of-life care delivery sporadic? A quantitative look at the barriers to and facilitators of providing end-of-life care in the neonatal intensive care unit. Advances in Neonatal Care, 11(1), 29-36.

doi: 10.1097/ANC.0b013e3182085642 
Wood, J. \& Milo, E. (2001). Fathers' grief when a disabled child dies. Death Studies, 25(8), 635661. doi:10.1080/713769895

Worden, J. (2002). Grief counseling and grief therapy: A handbook for the mental health practitioner, $3^{\text {rd }}$ ed. New York, New York: Springer.

Workman, E. (2001). Guiding parents through the death of their infant. Journal of Obstetrical, Gynecological, and Neonatal Nursing, 30(6), 569-573. doi: 10.1111/j.15526909.2001.tb00003.x

Yam, B. M. C., Rossiter, J. C., \& Cheung, K. Y. S. (2001). Caring for dying infants: Experiences of neonatal intensive care nurses in Hong Kong. Journal of Clinical Nursing, 10(5) ,. 651-9. doi: 10.1046/j.1365-2702.2001.00532.x

Yoon, P., Olney, R., Khoury, M., Sappenfield, W., Chavez, G., \& Taylor, D. (1997). Contribution of birth defects and genetic diseases to pediatric hospitalizations. A population-based study. Archives of Pediatric and Adolescent Medicine, 151, 1096-1103. Retrieved from http://archpedi.jamanetwork.com/journal.aspx

Zwerdling, T., Davies, S., Lazar, L., Crawford, B., Tucker, L., Boughner, A. \& Richter-Beck, L. (2000). Unique aspects of caring for dying children and their families. American Journal of Hospice and Palliative Care, 17(5), 305-311. doi:10.1177/104990910001700507 


\title{
Appendix A
}

\section{WestVirginiaUniversity}

\author{
School of Nursing
}

Dear Research Participant:

Thank you for your interest in the research study entitled A Grounded Theory Study of Parenting an Infant with a Life-Limiting Congenital Anomaly. Susan Welch, PhD candidate is conducting the study under the direction of Alvita Nathaniel, PhD, Assistant Professor in the School of Nursing at West Virginia University.

The purpose of this study is to learn more about the experience of being the parent of a child who died with a life-limiting congenital anomaly. You must be 18 years of age or older to participate. Participation in this study is voluntary. If you decide to participate, you may refuse to answer any question or may discontinue the interview at any time. If you experience sad or unhappy feelings and would like to talk to a counselor after the interview, Susan Welch will be glad to offer you a telephone number or referral for counseling services.

All information about you obtained from your participation will be kept confidential. No information that can identify you will be kept with the data that is being collected. In any publications that result from this research, neither your name nor any information from which you might be identified will be published without your consent.

If you have questions about this research, contact Susan Welch at 304-696-2631 (office), 304-638-8215 (cell) or welchs@marshall.edu or Dr. Alvita Nathaniel at (304) 347-1224 or anathaniel@hsc.wvu.edu. For more information about your rights as a research participant, you can also contact the Executive Secretary of the West Virginia University Institutional Review Board at 304-293-7073.

An acknowledgement of this research protocol is on file in the offices of the West Virginia University Institutional Review Board.

Thank you for you willingness to participate.

Sincerely:

Susan Welch, MS,RN

Research Office

6400 Health Sciences South PO Box 9640

Morgantown. WV 26506-9640
Equal Opportunity/Affirmative Action Institution 


\title{
Appendix B
}

\section{West VurginiaUniversity. \\ Office of Research Compliance}

\section{Expedited-IRB Protocol-Exemption}

\author{
To: $\quad$ Nathaniel, Alvita \\ From: WVU Office of Research Compliance \\ Date: $\quad$ Tuesday, March 09, 2010 \\ Subject: Exemption Acknowledgement
}

Tracking \#: $\mathrm{H}-22253$

Title: $\quad$ A Grounded Theory Study of Parenting an Infant with a Life-Limiting Congenital Anomaly

The above-referenced study was reviewed by the West Virginia University Institutional Review Board (IRB) and was granted exemption in accordance with 45 CFR 46.101(2).

This protocol was reviewed using the following:

Exemption Checklist (210r)

This research study was granted an exemption in accordance with Research that involves educational tests, survey procedures, interview procedures or observation of public behavior [45 CFR 46.101(2)].

The following documents have been acknowledged for use in this study and are available in the BRAAN system:

Locations of Research

Offsite Location Details

Attachments - Other (e.g., community hospital, nursing home, long-term care facility, private residence, etc.)

irb offsite location[1].doc Explanation of off-site site.

Surveys, Questionnaires, Interviews

Attachments

irb demographic form[1][1].doc

irb interview guide.doc

Advertisements

Advertisement 
Attachments - Newspaper recruitment ad dissertation recruitment ad.doc Newspaper

Miscellaneous Attachments

Attachments

Dissertation referral list.doc A list a bereavement service providers for participants of the study.

dissertation study letterhead letter[1].doc Letter explaining the study to participants

Thank you.

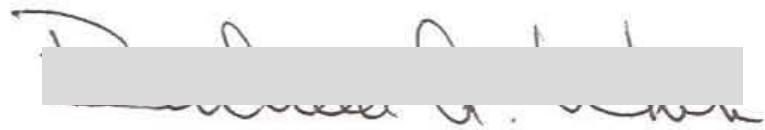

Board Designee: White, Barbara

Letter Sent By: White, Barbara, 3/9/2010 10:17 AM

Once you begin your human subject research, the following regulations apply:

1. Any modifications to the study protocol must be reviewed and acknowledged by the IRB prior to implementation.

2. You may not use a modified form until it has been acknowledged by the IRB. 


\title{
IRB PROTOCOL
}

\author{
Tracking \#: $\quad \mathrm{H}-22253$ \\ Version 2 \\ Exempt \\ RESEARCH TEAM \\ PRIMARY STUDY CONTACT \\ Is someone from the research team, other than the $\mathrm{PI}$, to be designated as the \\ primary contact for communications with the IRB (e.g. research nurse, study \\ coordinator, $\mathrm{PI}$, etc)? \\ Yes \\ PRIMARY CONTACT \\ Primary Contact Name: \\ Welch, Susan \\ Email: \\ swelch1@mix.wvu.edu \\ Mailbox \#: \\ (no answer) \\ Phone: \\ 304-733-0117 \\ Fax: \\ (no answer) \\ Department: \\ (Select only one. If you do not see your department in the list, please contact the \\ Research Compliance Office.) \\ If your school has departments, click on the + beside the school to view the list \\ of departments. Do not put a check in the school box if there are departments in \\ the school. \\ NURSING - Research \\ CONFLICT OF INTEREST \\ Do you or your immediate family have a financial \\ interest related to the research? \\ No \\ If Yes, attach a completed WVU Conflict of Interest Ad Hoc Disclosure Form (Fo \\ und Here). If available also attach an approval from the WVU Conflict of Interest \\ Committee and an approved Conflict Management Plan. - file attachment \\ - (no attachments)
}


IRB PROTOCOL

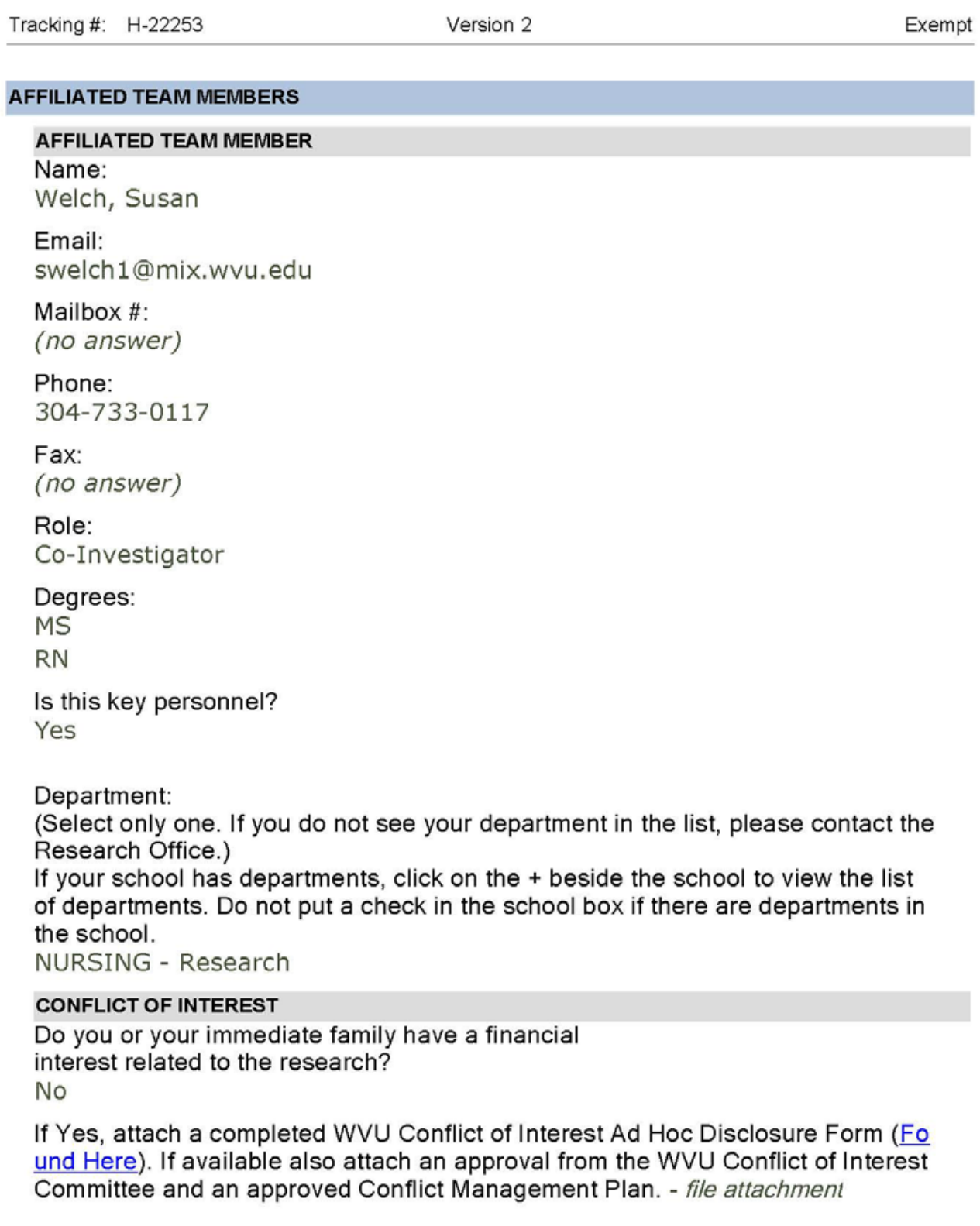




\section{IRB PROTOCOL}

Tracking \#: $\quad \mathrm{H}-22253$

Version 2

Exempt

- (no attachments)

Page 5 of 29 


\section{IRB PROTOCOL}

Tracking \#: $\mathrm{H}-22253$

Version 2

Exempt

\section{HUMAN SUBJECT RESEARCH}

HUMAN SUBJECTS RESEARCH

The following are NOT considered research:

- Class Projects

- Single Case Studies

- Program Evaluation, Quality Improvement, Needs Assessment

Does the protocol meet the federal definition of "Research"? OHRP Human Subject

Regulations Decision Charts

Yes

RESEARCH ON LIVING PERSONS

Does the research involve living person(s)?

Yes

COLLECTION OF INDIVIDUAL INFORMATION

Are you collecting information from individual person(s) as part of this research?

Yes 


\section{IRB PROTOCOL}

Tracking \#: $\mathrm{H}-22253$

Version 2

Exempt

\section{FUNDING SOURCE}

IRB FEE

WVU charges for IRB review for all projects that are supported by an industry sponsor. For the IRB Fee Schedule Click Here. For invoicing infomation please contact the Research Compliance office at 293-7073.

Has IRB fee been paid?

Not Applicable

FUNDING SOURCE(S)

Is there funding available for this research protocol?

No

Are there any grants for this research?

No

Attach any grant applications - file attachment

- (no attachments) 


\section{IRB PROTOCOL}

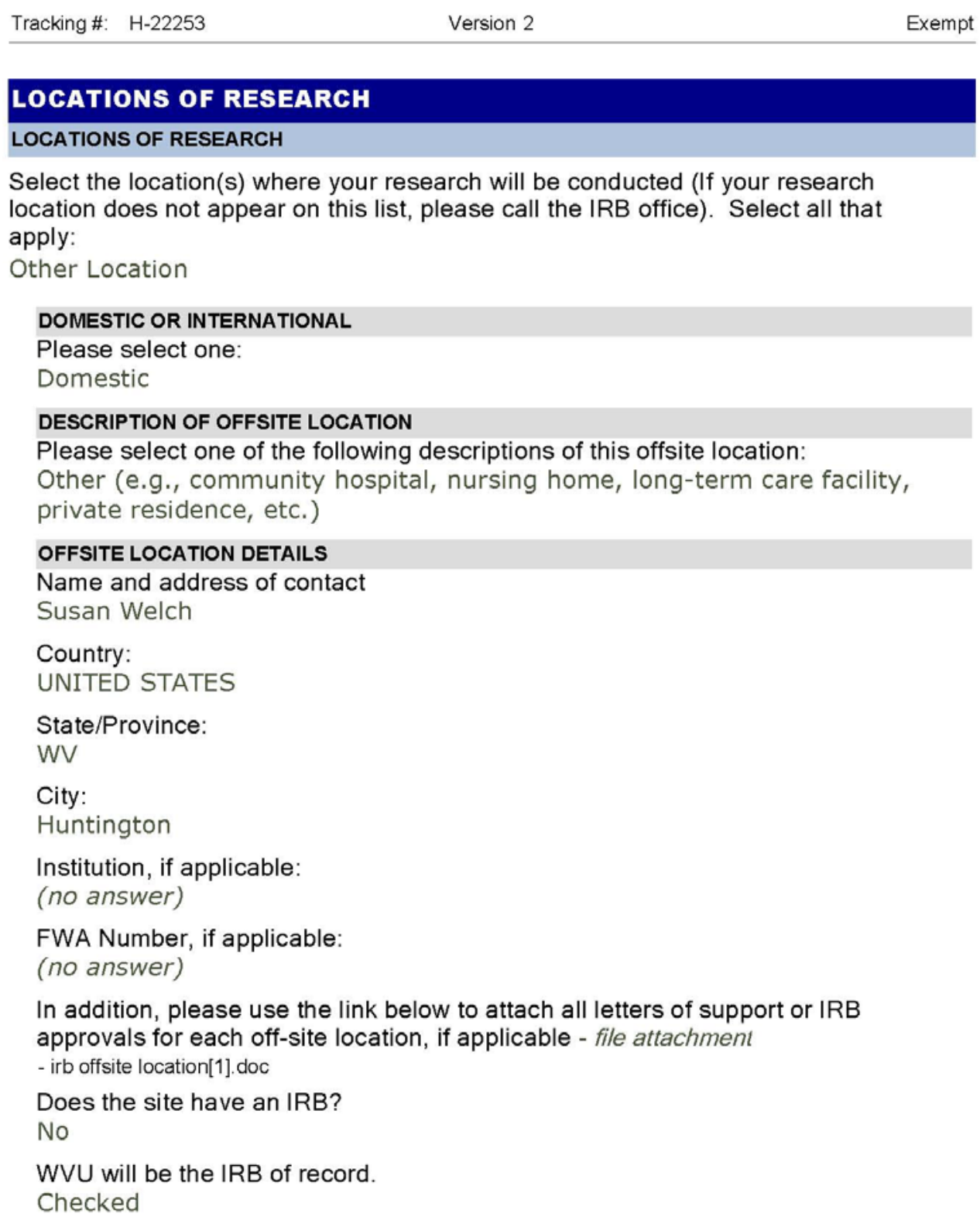


IRB PROTOCOL

\section{FACILITY}

Decribe how your facility is adequate to conduct this research. Include details on protecting participant safety, confidentiality, and privacy as appropriate.

The participant may chose the chose the site of the interview. Anticipated sites may include the participant's home, hospice conference room, church parlor, or public library conference room. The research associate will arrange meeting sites other than the participant's home to maintain confidentiality for the participant. Privacy will be maintained as all interviews will occur behind closed doors with only the research associate and participant present.

Describe the availability of medical or psychological resources that participants might need as a consequence of the research.

The research associate will suggest the participant contact their clergy, a counselor at the nearest hospice or a perinatal loss support group as psychological resources should the need arise. 


\section{IRB PROTOCOL}

Tracking \#: $\quad H-22253$

Version 2

Exempt

\section{EXEMPTION DETERMINATION}

EXEMPT FROM IRB REVIEW

Select all that apply:

Research that involves educational tests, survey procedures, interview procedures, or observation of public behavior [45 CFR 46.101(2)]

Describe how your research meets the requirements of the exemption category you selected above:

This study involves unstructured interviews with participants. No data indentifying the participant will be retained with the field notes. Inadvertant identification of a participant would not pose any legal, social or financial risk to the participant 


\section{IRB PROTOCOL}

\section{LAY SUMMARY}

LAY SUMMARY

Provide a lay summary (6th grade reading level) for this study:

End-of-life care for infants and very young children who die of lethal birth defects and their families is less than optimal. Little research has explored the experience of families as they care for an infant who will live only a short time. Parent(s) who had a baby die of from a birth defect will be asked to talk about their experience as the parent of that baby. The research associate will create notes after the interview and later will look for common themes in those notes. 


\section{IRB PROTOCOL}

\section{PURPOSE AND OBJECTIVES}

PURPOSE AND OBJECTIVES

What is the research question? What are the specific aims?

The purpose of this research is to increase understanding of the process of being the parent of an infant that will die of a life-limiting congenital anomaly within the first year of life. A substantive theory will be outcome of the research. That theory then can help health care providers develop and deliver appropriate interventions and resources for parents who are experiencing or experienced the death of an infant from life-limiting congenital anomalies. 


\section{IRB PROTOCOL}

Version 2

Exempt

\section{DESIGN}

DESIGN

Categorize your research (Select all that apply):

Surveys, etc. - Interview

Surveys, etc.

Is this a Clinical Trial?

No

Page 13 of 29 
IRB PROTOCOL

Include a literature review and explain the scientific rationale for the study.

Every year about 27,000 neonates and infants die in the United States with congenital anomalies as the leading cause of death (Field \& Behrman, 2003; National Hospice and Palliative Care Organization, 2001). A very small percentage of dying babies receive palliative or end-of-life services and most die in intensive care units ( Feudtner et al, 2002; Last Acts, 2002; Leuthner, Boldt, \& Kirby, 2004; Zwerdling et al, 2000). Inadequate palliative care negatively affects the infant, families and society because of increased pain and suffering as well as increased direct and indirect economic costs (Adams, Corr, Davies, \& Deveau, 1999; Belasco, Danz, Drill, Schmid, \& Burkey, 2000; Black, 1998; Costello \& Trinder-Brook, 2000; Feudtner, DiGiuseppe, \& Neff, 2003; Kazak \& Noll, 2004; Li et al, 2003; NHPCO, 2001; Papdatou et al, 2002;

Pierucci, Kirby \& Leuthner, 2001; Wood \& Milo, 2001; Yam, Rossiter \& Cheung, 2001).

Few authors address palliative and end - of - life care for neonates and infants. Most of the current literature arises from professional experience and includes descriptions of the basic components of infant palliative and end - of - life care, informally written "how to's", and formal extensive protocols (Caitlin \& Carter, 2002; Carter \& Bhatia, 2001; Crag \& Goldman, 2003; Gale \& Brooks, 2006; Romesburg, 2007; Toce, 2000; Walden, Sudi-Robinson \& Carrier, 2001). Research literature is scant. Ventilator withdrawal was the most common topic related to dying infants. One group of researchers at University of Wisconsin Children's Hospital explored palliative care for dying infants in three different studies( Feudtner, DiGiuseppe, \& Neff, 2003; Feudtner et al, 2002; Pierucci, Kirby, \& Leuthner, 2001).

The literature that specifically explores palliative and end - of - life care for infants dying of life - limiting congenital anomalies and their families is rarer. Leuthner (2004) wrote on the provision of palliative care for this group of infants. He suggested that palliative care is an option whether diagnosis of the lethal anomaly occurs prior to and after delivery.

Parents of infants with life-limiting congenital anomalies, as primary providers of love and care, are a vital part of any discussion regarding optimal palliative and end-of-life care. Yet only Brosig et al, 2007 explored the experience of parents whose infant died of a life-limiting congenital anomaly. There exists a significant knowledge gap. The purpose of this study is to a) explore the process of parenting as parents experience the birth, life and ultimate death of an infant from a life-limiting congenital anomaly and b) develop a substantive theory to describe the parental process from birth to death. Sound evidence will allow health care providers to tailor effective interventions to allow for provision of a good death for both the infant and their families. 


\section{IRB PROTOCOL}

Discuss the research design including but not limited to such issues as: probability of group assignment, potential for subject to be randomized to placebo group, use of control subjects, etc.

This study will use Classic Grounded Theory methodology to identify themes from unstructured interview data. Convenience sampling will be employed. Personal/professional networks will be used to recruit participants.

Specify inclusion criteria:

Mothers and fathers, who are currently over 18, of live born infants who died of a life-limiting congenital anomaly. The infant must have lived 48 hours and died within the first 15 months of life. Participants must understand and speak English.

Specify exclusion criteria:

Parents who are under the age of 18 and do not speak or understand English. Mothers and fathers whose infant with an anomaly that was stillborn.

\section{PROTECTED HEALTH INFORMATION}

Does the research involve $\mathrm{PHI}$ in any way?

No

\section{RISKS TO PARTICIPANTS}

Does this research involve more than minimal risk to participants?

No

IF YES: Describe the provisions to monitor the data to ensure the safety of participants (data and safety monitoring plan.) Address theses questions: Who will monitor the data? What data will be monitored? How frequently will data be monitored? What analyses will be performed on the data? What decision rules (e.g., stopping rules) will be considered? Will unexpected harms be detected promptly? Will an increased frequency or severity of unexpected harms be detected promptly? Will the protocol be stopped once benefits are proven to outweigh harms? Will the protocol be stopped once harms are proven to outweigh benefits?

The participant(s) may experience sadness revisiting their experience of caring for and parenting their deceased infant. 


\section{IRB PROTOCOL}

\section{PROCEDURES}

Describe (in detail) all procedures to be performed in sequential order as they will be performed:

1. Obtain IRB approval 2. Seek participants 3. Potential participant contacts the research associate to discuss the purpose of the study and logistics of conduct of the interview. (Note: Classic Grounded Theory has 7 non-linear steps, but are presented in a linear manner) 4 . Meet with participant and conduct interview. 5. Write field notes. 6. Conduct theoretical coding of field notes 7. Write memos about emerging concepts related to codes and relationships. 8. Conduct theoretical sampling. 9. Write more memos. 10. Sort memos according to theoretical relationships. 11. Write grounded theory 


\section{IRB PROTOCOL}

\section{SURVEYS, QUESTIONNAIRES, INTERVIEWS}

SURVEYS, QUESTIONNAIRES, INTERVIEWS

Will you be asking any questions that might upset your subjects? (Examples are questions about abuse, trauma, etc.) If yes, put a counseling services referral list on the "Locations of Research" page in the Facility section where it asks for availability of resources. If you need to attach a separate list, please attach under "Miscellaneous Attachments".

No

Attach any and all surveys, questionnaires, interviews: - file attachment

- irb demographic form[1][1].doc

- irb interview guide doc 


\section{IRB PROTOCOL}

\section{FULL BOARD OR EXPEDITED REVIEW ASSESSMENT}

EXPEDITED REVIEW CATEGORIES

If you selected a category for Exemption, please be sure to choose Not Applicable under the expedited categories.

Select the expedited review category below:

Not Applicable - This research does not meet the requirements for any of the following categories.

Describe how your research meets the requirements of the category you selected above:

(no answer)

DECEPTION OF SUBJECTS

Will you be deliberately deceiving subjects as to the purpose of the study? No

If yes, describe how you will debrief the subjects after their participation is completed.

(no answer)

\section{SPECIAL REVIEW TYPES}

Has this research been reviewed by a WVU Scientific Review Committee?

No

If yes, then which one?

(no answer)

Is this research eligible for $\mathrm{NCl}$ Central IRB Review?

No

Attach Central IRB Application and approval: - file attachment

- (no attachments) 


\section{IRB PROTOCOL}

Version 2

Exempt

\section{IRB AUTHORIZATION AGREEMENT}

IRB AUTHORIZATION AGREEMENT

If you are collaborating with another institution, select the applicable statement regarding IRB Authorization Agreement below:

I am not requesting an IAA. 
IRB PROTOCOL

\section{SUBJECTS}

SUBJECTS

\section{Gender}

Both

Ages (Select all that apply)

$18+$ years

Describe in detail what will be done to identify and recruit participants.

Contact personal and professional acquaintances about their awareness of someone in their personal or professional network who meets the inclusion criteria. Ask the person to: a) contact the potential participant to discern interest in participation b) give the potential participant the research associate's contact information if they wish to participate. Contact the potential participant once interest is indicated by emailing or phoning the research associate.

The research associate plans to use snowballing to recruit additional participants. It is not unusual for people undergoing a challenging situation to reach out to others who experienced a similiar situation either in the past or currently. Therefore it is likely that participants know someone else who experienced the loss of an infant from a life-limiting congenital anomaly. The research associate may also advertise in newspapers within a 2 hour radius of her home for potential participants should limited or no participants be recruited in the above manner.

Finally, the research associate may contact the web master of on-line support groups for parents whose infants have life-limiting conditions seeking permission to advertise for participants.

\section{VULNERABLE POPULATIONS}

Vulnerable Populations (Select all that apply):

N/A

Vulnerable populations require special protections. How will you obtain informed consent, protect subject confidentiality, prevent undue coercion, and otherwise protect the rights and welfare of these participants?

(no answer) 


\section{IRB PROTOCOL}

Tracking \#: H-22253

Version 2

Exempt

\section{COERCION OR UNDUE INFLUENCE}

Does this research involve participants who could be coerced or unduly influenced? No

IF YES: Describe the additional provisions to protect their rights and welfare

(no answer)

\section{CHILDREN}

Does this research involve children?

No

Who will provide consent? (Select only one)

(no answer)

Will assent be obtained from all children?

(no answer)

IF NO:Explain

(no answer)

Will assent be documented using an assent form?

(no answer)

IF NO:Explain

(no answer)

\section{PRIMARY LANGUAGE}

Will the informed consent form be translated into (an)other language(s)?

No

Into what language(s) will the consent form be translated? (Select all that apply. To make multiple selections, hold down the CTRL key while clicking.)

(No Translation) 


\section{IRB PROTOCOL}

\section{SAMPLE SIZE}

SAMPLE SIZE

Number of subjects to be enrolled by WVU researchers:

20

Please indicate why you chose the sample size proposed.

20 participants should allow the research associate to reach saturation.

Is this a multi-site study?

No

DATA ANALYSIS

Provide a description of your plan for data analysis. How will the analyses proposed relate to the primary purposes of your study?:

In Classic Grounded Theory data analysis occurs simulataneously with data collection. The research associate will write field notes immediately after an interview. Open and theoretical coding of the data and memo writing follows. Sorting of the memos occurs once saturation occurs. Writing of the grounded theory is the "last"step of data analysis.

The outcome of the data analysis is a substantive theory which will assist health care professionals in provision of care based in evidence.

For more than minimal risk describe how data will ensure the safety of participants. Not applicable-Minimal risk 


\section{IRB PROTOCOL}

\section{POTENTIAL RISKS / DISCOMFORTS}

POTENTIAL RISKS / DISCOMFORTS

Describe and assess any potential possibilities for risk or harm to the subjects as a result of their participation in the research, including discomforts, hazards, or inconveniences to the subject.

The participant may experience sadness revisiting the experience of parenting their deceased infant.

Describe the provisions to protect the privacy interests of participants. "Privacy interest" is distinct from confidentiality and refers to the interest of individuals to be free from unwanted intrusions.

Participants are free to not answer any question or stop the interview at any time. 


\section{IRB PROTOCOL}

\section{POTENTIAL BENEFITS}

POTENTIAL BENEFITS

Describe potential benefit(s) to be gained by the individual subject as a result of participating in the research. (Payments to subjects should not be included in this section.)

Benefits to the participant include: 1 . The opportunity to share their story. Family and friends have heard the story many times and may not want to hear the story again. 2. The knowledge that sharing their story may help a parents facing a similiar situation in the future.

Describe the potential benefit(s) to society and scientific / medical knowledge of the planned work.

The information gained will potentially enable health care providers to better understand the experience of parents as they care for their infant with a life-limiting congenital anomaly. This knowledge will help health care providers develop evidence-based interventions to assist parents as they care for dying babies and the subsequent grief.

Discuss the risk-to-benefit ratio. Describe how benefits outweigh potential risks. Both the risk and benefits are psychological or emotional in nature with potential for either minimal. Sadness related to the death of the child is not created by the interview question, but rather by the experience itself. Participating may create a benefit if participants are able to share their story and think they are helping other parents. Therefore, the potential benefits outweight the potential risks. 


\section{IRB PROTOCOL}

Tracking \#: $\quad H-22253$

Version 2

Exempt

\section{CONFIDENTIALITY}

IDENTIFIABLE

Is there any way subjects can be associated with the data?

No

State what steps will be taken to maintain confidentiality of data and privacy of subjects:

Confidentiality: Demographic information, field notes and memos will be stored on the research associate 's personal password protected computer or filed in a locked file cabinet in her office. No names will be used in any subsequent publications from the study.

Privacy: Interviews will occur in private settings. No one other than the research associate will have the participant's contact information.

Do you have a Federal Certificate of Confidentiality for this study? No

Attach Federal Certificate of Confidentiality or pending application: - file attachment - (no attachments) 


\section{IRB PROTOCOL}

Version 2

Exempt

COSTS TO SUBJECTS

COSTS TO SUBJECTS

Will this research cost the subjects anything?

No

Page 26 of 29 


\section{IRB PROTOCOL}

Tracking \#: $\quad \mathrm{H}-22253$

Version 2

Exempt

\section{PAYMENTS}

PAYMENTS

Will subjects be paid (money, gift certificates, coupons, etc.) to participate in this research project?

No

If yes, please note the total dollar amount (or dollar amount value) of the payment: (no answer)

If yes, please note the distribution plan (one payment, pro-rated payment, paid upon completion, etc.):

(no answer)

\section{EXTRA CLASS CREDIT}

Will students receive extra class credit for participating in this research project? No

Only the following school(s)/department have IRB approval for extra credit. Select Department(s):

(no answer) 


\section{IRB PROTOCOL}

\begin{tabular}{llll} 
Tracking \#: $\mathrm{H}-22253$ & Version 2 & Exempt \\
\hline
\end{tabular}

\section{ADVERTISEMENTS}

\section{ADVERTISEMENTS}

Will you advertise for this study?

Yes

\section{ADVERTISEMENT NAME}

Enter a name for the advertisement:

Newspaper recruitment ad

\section{ADVERTISEMENT}

Exact language of advertisement:

See attached file.

Attach advertisement, if applicable - file attachment

- dissertation recruitment ad. doc

Describe where ads will be placed.

Ad will be placed in the classified ad section of newspapers within 2 hours drive of research associate's home.

Web sites are another potential site for posting of the advertisement. 


\section{IRB PROTOCOL}

Tracking \#: $\quad \mathrm{H}-22253$

Version 2

Exempt

MISCELLANEOUS ATTACHMENTS

MISCELLANEOUS ATTACHMENTS APPROVAL

Click to attach document submitted for Approval - file attachment

- dissertation study letterhead letter[1].doc

- Dissertation referral list.doc

\section{MISCELLANEOUS ATTACHMENTS INFORMATIONAL}

Click to attach document for Informational Purposes - file attachment

- (no attachments) 